\title{
Confirming the existence of the strong CP problem in lattice QCD with the gradient flow
}

\author{
Jack Dragos, ${ }^{1}$ Thomas Luu, ${ }^{2}$ Andrea Shindler $\odot,{ }^{1}$ Jordy de Vries, ${ }^{3,4}$ and Ahmed Yousif ${ }^{1}$ \\ ${ }^{1}$ Facility for Rare Isotope Beams, Physics Department, Michigan State University, East Lansing, Michigan 48824, USA \\ ${ }^{2}$ Institute for Advanced Simulation (IAS-4), Institut für Kernphysik (IKP-3) and JARA-HPC, FZJ, Jülich, Germany \\ ${ }^{3}$ Amherst Center for Fundamental Interactions, Department of Physics, University of Massachusetts Amherst, \\ Amherst, Massachusetts 01003, USA \\ ${ }^{4}$ RIKEN BNL Research Center, Brookhaven National Laboratory, Upton, New York 11973-5000, USA
}

(Received 28 April 2019; revised 18 May 2020; accepted 22 July 2020; published 19 January 2021)

\begin{abstract}
We calculate the electric dipole moment of the nucleon induced by the quantum chromodynamics $\theta$ term. We use the gradient flow to define the topological charge and use $N_{f}=2+1$ flavors of dynamical quarks corresponding to pion masses of 700,570 , and $410 \mathrm{MeV}$, and perform an extrapolation to the physical point based on chiral perturbation theory. We perform calculations at three different lattice spacings in the range of $0.07 \mathrm{fm}<a<0.11 \mathrm{fm}$ at a single value of the pion mass, to enable control on discretization effects. We also investigate finite-size effects using two different volumes. A novel technique is applied to improve the signal-tonoise ratio in the form factor calculations. The very mild discretization effects observed suggest a continuumlike behavior of the nucleon electric dipole moment toward the chiral limit. Under this assumption our results read $d_{n}=-0.00152(71) \bar{\theta} e \mathrm{fm}$ and $d_{p}=0.0011(10) \bar{\theta} e \mathrm{fm}$. Assuming the $\theta$ term is the only source of CP violation, the experimental bound on the neutron electric dipole moment limits $|\bar{\theta}|<1.98 \times 10^{-10}$ (90\% CL). A first attempt at calculating the nucleon Schiff moment in the continuum resulted in $S_{p}=0.50(59) \times 10^{-4} \bar{\theta} e \mathrm{fm}^{3}$ and $S_{n}=-0.10(43) \times 10^{-4} \bar{\theta} e \mathrm{fm}^{3}$.
\end{abstract}

DOI: 10.1103/PhysRevC.103.015202

\section{INTRODUCTION}

A nonzero measurement of the electric dipole moment (EDM) of the nucleon in the foreseeable future would be a clear signal of new physics, since the known CP-violating phase of the Cabibbo-Kobayashi-Maskawa (CKM) matrix leads to EDMs that lie orders of magnitude below current experimental limits. The source of a nonzero EDM could then either be the quantum chromodynamics (QCD) $\bar{\theta}$ term or higher-dimension $\mathrm{CP}$-violating quark-gluon operators that originate in beyond-the-standard model (BSM) physics, or a combination of these two. To interpret an EDM signal or lack thereof, and to possibly disentangle the source (e.g., $\bar{\theta}$-term or BSM), requires a nonperturbative calculation linking the $\mathrm{CP}$-violating sources to the hadronic observables.

Lattice QCD can calculate the nucleon EDM directly in terms of CP-violating operators at the quark level. Various attempts have been made in this regard [1-7]. However, the renormalization of $\mathrm{CP}$-violating operators within a lattice (discretized) formulation of QCD is very nontrivial, and for

Published by the American Physical Society under the terms of the Creative Commons Attribution 4.0 International license. Further distribution of this work must maintain attribution to the author $(s)$ and the published article's title, journal citation, and DOI. Funded by $S C O A P^{3}$. several operators presents large difficulties in interpreting lattice results. Further, the $\bar{\theta}$ term itself introduces a complex phase in the determinant of the quark matrix, which produces a sign problem and precludes the use of standard stochastic methods. Several techniques have been used to address the $\bar{\theta}$-term contribution to the EDM and attempts have been made to solve the complicated renormalization patterns of the CPviolating operators [8]. We refer to the recent review [9] for a summary.

We proposed to use the gradient flow to calculate all CPviolating source to the EDM in Refs. [10,11], and presented preliminary results [12-15]. We have also performed a first perturbative study of the flow-time dependence of the quarkchromo and Weinberg operators [16].

In this paper we consider the $\bar{\theta}$-term contribution to the EDM in a perturbative manner as discussed in Ref. [11]. This is well justified considering the stringent constraints on $\bar{\theta}$ set by EDM experiments. In this way we avoid the problem of a complex fermionic determinant. To define the QCD $\bar{\theta}$ term we use the gradient flow. The topological charge defined in this way has a finite and well defined continuum limit [17-19]. It is much faster to compute than using the Ginsparg-Wilson definition and it is theoretically more robust than definitions using cooling techniques. Another problem that hinders lattice calculations of the nucleon EDM is the very poor signalto-noise ratio. In this respect we explore a novel technique to determine the space-time region where the signal in the relevant correlation functions is maximized. A first account of this technique has already been presented [12]. 
Additional insights into the EDM of the nucleon [20-24] and nuclei $[25,26]$ can be provided by chiral effective field theory. The CP-violating quark operators are translated to effective CP-violating hadronic operators and EDMs depend on the unknown low-energy constants (LECs) of the theory. The LECs can be estimated from dimensional analysis or, preferably, be determined from experiments and/or by lattice-QCD calculations. We use these insights from chiral calculations to understand the pion mass dependence of our results, and to connect our nucleon EDM calculations to nuclear EDMs.

The remainder of the paper is organized as follows: Section II gives a cursory discussion of the phenomenology of the nucleon EDM, followed by an overview of the lattice details and parameters in Sec. III, where we discuss our general lattice strategy and we define the basic observables, including the gradient flow. Sanity checks are given in Sec. IV, where we compute and display the topological charge using the gradient flow. The nucleon two-point correlation function is explored in Sec. V, then the setup of the computation of the EDM is derived and results shown in Sec. VI. A discussion follows the results section in Sec. VII, where we discuss the ramifications of our results and compare our results to the literature. Finally, we conclude in Sec. VIII.

\section{PHENOMENOLOGY OF THE QCD THETA TERM}

The discrete space-time symmetries parity $(P)$ and timereversal $(T)$, and hence via the $C P T$ theorem also $C P$ symmetry, are broken in QCD by the $\bar{\theta}$ term. In the case of two quark flavors the QCD Lagrangian in Minkowski space is given by

$\mathcal{L}_{\mathrm{QCD}}=-\frac{1}{4} G_{\mu \nu}^{a} G^{a, \mu \nu}+\bar{q}(i \not D-M) q-\bar{\theta} \frac{g^{2}}{64 \pi^{2}} \epsilon^{\mu \nu \alpha \beta} G_{\mu \nu}^{a} G_{\alpha \beta}^{a}$,

where $q=(u, d)^{T}$ denotes the quark doublet containing up and down quarks, $G_{\mu \nu}^{a}$ is the gluon field strength tensor, $\epsilon^{\mu \nu \alpha \beta}\left(\epsilon^{0123}=+1\right)$ is the completely antisymmetric tensor, $D_{\mu}$ the gauge-covariant derivative, $M$ the real $2 \times 2$ quarkmass matrix, and $\bar{\theta}$ the coupling of the $C P$-odd interaction. In Eq. (1) the complex phase of the quark-mass matrix has been absorbed in the physical parameter $\bar{\theta}=\theta+\arg \operatorname{det}(M)$. For the application of chiral perturbation theory $(\chi \mathrm{PT})$ it is useful to perform an anomalous axial $U(1)$ transformation to replace the $C P$-odd gluonic term in favor of a complex mass term $[20,27]$. Under the assumption that $\bar{\theta} \ll 1$ the QCD Lagrangian can then be written as

$$
\mathcal{L}_{\mathrm{QCD}}=-\frac{1}{4} G_{\mu \nu}^{a} G^{a, \mu \nu}+\bar{q} i \not D q-\bar{m} \bar{q} q-\varepsilon \bar{m} \bar{q} \tau_{3} q+m_{*} \bar{\theta} \bar{q} i \gamma^{5} q,
$$

where we have defined the average quark mass $\bar{m}=\left(m_{u}+\right.$ $\left.m_{d}\right) / 2$, the quark-mass difference $\varepsilon=\left(m_{u}-m_{d}\right) /\left(m_{u}+m_{d}\right)$, and the reduced quark mass $m_{*}=m_{u} m_{d} /\left(m_{u}+m_{d}\right)=\bar{m}(1-$ $\left.\varepsilon^{2}\right) / 2$.

The QCD $\bar{\theta}$ term induces an EDM in hadrons and nuclei. The first EDM search occurred with the neutron in 1957 $[28,29]$. Till this day, no signal has been found for the EDM, despite measurement sensitivities having improved by six orders of magnitude. The current bound $d_{n}<3.0 \times 10^{-26} \mathrm{e} \mathrm{cm}$ [30,31] sets strong limits on the size of $\bar{\theta}$ and sources of $C P$ violation from physics beyond the SM [32].

To set a bound on the $\bar{\theta}$ term, it is necessary to calculate the dependence of the neutron EDM on $\bar{\theta}$ [20]. One way to do this is by using $\chi \mathrm{PT}$. In the first step one derives interactions between the low-energy degrees of freedom, pion and nucleons (and heavier hadrons), that violate $C P$ and transform the same way under chiral symmetry as the complex mass term in Eq. (2). In the second step, one combines the chiral $C P$-odd interactions with the standard $C P$-even chiral Lagrangian to calculate the nucleon EDM. This calculation has been done up to next-to-leading order (NLO) in both $S U(2)$ and $S U(3) \chi \mathrm{PT}$ $[21,23]$ and gives in the two-flavored theory for the neutron $\left(d_{n}\right)$ and proton $\left(d_{p}\right) \mathrm{EDM}$ :

$$
\begin{aligned}
& d_{n}(\theta)=\bar{d}_{n}-\frac{e g_{A} \bar{g}_{0}}{8 \pi^{2} F_{\pi}}\left(\ln \frac{m_{\pi}^{2}}{m_{N}^{2}}-\frac{\pi m_{\pi}}{2 m_{N}}\right), \\
& d_{p}(\theta)=\bar{d}_{p}+\frac{e g_{A} \bar{g}_{0}}{8 \pi^{2} F_{\pi}}\left(\ln \frac{m_{\pi}^{2}}{m_{N}^{2}}-\frac{2 \pi m_{\pi}}{m_{N}}\right),
\end{aligned}
$$

in terms of $g_{A} \simeq 1.27$ the strong pion-nucleon coupling constant, $F_{\pi} \simeq 92.4 \mathrm{MeV}$ the pion decay constant, $m_{\pi}$ and $m_{N}$ the pion and nucleon mass respectively, $e>0$ the proton charge, and three low-energy constants (LECs) of $C P$-odd chiral interactions $\bar{g}_{0}$ and $\bar{d}_{p / n}$. The first term in brackets in Eq. (3) arises from the leading-order (LO) one-loop diagram involving the $C P$-odd vertex,

$$
\mathcal{L}_{\pi N}(\theta)=\bar{g}_{0} \bar{N} \vec{\pi} \cdot \vec{\tau} N
$$

in terms of the nucleon doublet $N=(p n)^{T}$ and the pion triplet $\vec{\pi}$. The LO loop is divergent and the divergence and associated scale dependence have been absorbed into the counter terms $\bar{d}_{p / n}$ which signify contributions to the nucleon EDMs from short-range dynamics and appear at the same order as the LO loop diagrams. The second term in brackets in Eq. (3) arises from finite next-to-leading-order (NLO) diagrams.

Because the $\bar{\theta}$ term breaks chiral symmetry as a complex quark mass, the LEC $\bar{g}_{0}$ can be related to known $C P$-even LECs using chiral symmetry arguments [20,33,34],

$$
\bar{g}_{0}=\frac{\left(m_{n}-m_{p}\right)^{\text {strong }}\left(1-\varepsilon^{2}\right)}{4 F_{\pi} \varepsilon} \bar{\theta}=-14.7(2.3) \times 10^{-3} \bar{\theta},
$$

where $\left(m_{n}-m_{p}\right)^{\text {strong }}$ is the quark-mass-induced part of the proton-neutron mass splitting for which we used the recent lattice results [35,36]. Inserting Eq. (68) into Eq. (3) we obtain

$$
\begin{aligned}
& d_{n}(\theta)=\bar{d}_{n}-2.1(3) \times 10^{-3} \bar{\theta} e \mathrm{fm}, \\
& d_{p}(\theta)=\bar{d}_{p}+2.5(3) \times 10^{-3} \bar{\theta} e \mathrm{fm} .
\end{aligned}
$$

Under the assumption that the terms $\bar{d}_{p / n}$ do not cancel against the calculable loop contributions, a comparison with the experimental bound gives the strong constraint $\bar{\theta} \leqslant 10^{-10}$. Clearly, a more reliable constraint on $\bar{\theta}$ requires a direct 
TABLE I. Summary of the lattice bare parameters for the ensembles used. $N_{G}$ is the number of gauge configurations and $N_{\text {corr }}$ is the number of correlation functions calculated using many stochoastically located sources for the same gauge configuration.

\begin{tabular}{ccccccccc}
\hline \hline & $\beta$ & $\kappa_{l}$ & $\kappa_{s}$ & $\mathrm{~L} / \mathrm{a}$ & $\mathrm{T} / \mathrm{a}$ & $\mathrm{c}_{\mathrm{sw}}$ & $N_{G}$ & $N_{\text {corr }}$ \\
\hline $\mathrm{M}_{1}$ & 1.90 & 0.13700 & 0.1364 & 32 & 64 & 1.715 & 322 & 30094 \\
$\mathrm{M}_{2}$ & 1.90 & 0.13727 & 0.1364 & 32 & 64 & 1.715 & 400 & 20000 \\
$\mathrm{M}_{3}$ & 1.90 & 0.13754 & 0.1364 & 32 & 64 & 1.715 & 444 & 17834 \\
$\mathrm{~A}_{1}$ & 1.83 & 0.13825 & 0.1371 & 16 & 32 & 1.761 & 800 & 15220 \\
$\mathrm{~A}_{2}$ & 1.90 & 0.13700 & 0.1364 & 20 & 40 & 1.715 & 789 & 15407 \\
$\mathrm{~A}_{3}$ & 2.05 & 0.13560 & 0.1351 & 28 & 56 & 1.628 & 650 & 12867 \\
\hline \hline
\end{tabular}

nonperturbative calculation of the full nucleon EDMs. This is the main goal of this work.

In the isoscalar combination $d_{n}+d_{p}$ the loop contribution cancels out to a large extent. For observables sensitive to this combination, such as the deuteron EDM whose measurement is the goal of the JEDI collaboration [37], a first-principle calculation of the total nucleon EDM is important. EDMs of light nuclei have been calculated as a function of $\bar{\theta}$ in Ref. [26]. Nuclear EDMs get contributions from the singlenucleon EDMs and from the CP-violating nucleon-nucleon potential which is dominated by one-pion-exchange terms. The latter depend mainly on $\bar{g}_{0}$ and are therefore relatively well under control. The dominant remaining uncertainty is the size of the nucleon EDMs as a function of $\bar{\theta}$. With nonperturbative calculations of nucleon EDMs induced by the $\bar{\theta}$ term, we immediately obtain predictions for EDMs of light nuclei. With future improvements of nuclear theory even EDMs of diamagnetic atoms such as ${ }^{199} \mathrm{Hg}$ and ${ }^{225} \mathrm{Ra}$ could be directly given as a function of $\bar{\theta}$.

\section{LATTICE QCD ACTION AND NUMERICAL DETAILS}

We discretize the QCD action on an hypercubic lattice with spacing $a$ and volume $L^{3} \times T$. The fermionic part of our QCD lattice action is the nonperturbatively $\mathrm{O}(a)$-improved Wilson action with $N_{f}=2+1$ dynamical quarks. The gauge part is the Iwasaki gauge action. For our calculation we always use valence quarks with the same lattice action and the same bare parameters as the sea quark action, that is to say our framework is fully unitary.

We performed calculations using the publicly available PACS-CS gauge fields available through the ILDG [38]. We used six different ensembles that allow us to study discretization effects, finite-size effects and pion mass dependence. We studied the pion-mass dependence with three ensembles at three different bare quark masses, at $L / a=32$ and $T / a=64$ and a lattice spacing $a=0.0907(13) \mathrm{fm}$. The lattice spacing and the physical point were determined with the experimental input of $m_{\pi}, m_{K}$, and $m_{\Omega}$. More details on these ensembles are available in Ref. [39] and are summarized in the first three $M$ rows of Tables I and II.

To study discretization effects we used three ensembles with three different lattice spacings but with the same volume, $L \simeq 1.8 \mathrm{fm}$. The ratios of masses in the pseudoscalar and vector channels differ, between the three ensembles, at most
TABLE II. Summary of some basic lattice quantities computed on the ensembles used.

\begin{tabular}{lcccc}
\hline \hline & $a[\mathrm{fm}]$ & $m_{\pi}[\mathrm{MeV}]$ & $m_{N}[\mathrm{GeV}]$ & $Z_{V}$ \\
\hline $\mathrm{M}_{1}$ & $0.0907(13)$ & $699.0(3)$ & $1.585(2)$ & $0.7354(37)$ \\
$\mathrm{M}_{2}$ & $0.0907(13)$ & $567.6(3)$ & $1.415(3)$ & $0.7354(37)$ \\
$\mathrm{M}_{3}$ & $0.0907(13)$ & $409.7(7)$ & $1.219(4)$ & $0.7354(37)$ \\
$\mathrm{A}_{1}$ & $0.1095(25)$ & $710(1)$ & $1.65(1)$ & $0.7013(14)$ \\
$\mathrm{A}_{2}$ & $0.0936(33)$ & $676.3(7)$ & $1.549(6)$ & $0.7354(37)$ \\
$\mathrm{A}_{3}$ & $0.0684(41)$ & $660.4(7)$ & $1.492(5)$ & $0.77314(82)$ \\
\hline \hline
\end{tabular}

by $1 \%$ in the light sector and at most of $3 \%$ in the strange quark sector. These very small mismatches are irrelevant for all purposes for our scaling violation study. The lattice spacings and quark masses in these ensembles are also determined using $m_{\pi}, m_{K}$, and $m_{\phi}$. Details for these ensembles can be found in Ref. [40] and summarized in the last three A rows of Tables I and II.

Among the six ensembles described above there are two ensembles, $\mathrm{M}_{1}$ and $\mathrm{A}_{2}$, with the same bare parameters, $\beta=$ $1.9, \kappa_{l}=0.13700, \kappa_{s}=0.1364$ and different lattice volumes with $L / a=20, L / a=32$, and $T=2 L$. These two ensembles allow us to investigate finite-size effects.

To improve the overlap with the ground state of the relevant matrix elements in the two- and three-point functions, we applied a Gaussian gauge-invariant smearing [41,42] at the source and at the sink of our quark propagators. Using the notation of Refs. [41,42] we use 64 iterations of the smearing algorithm with a smearing fraction of $\alpha=0.39$ using the definition in Ref. [41]. These parameters corresponds to a spatial root-mean-square radius for the nucleon interpolating operator of around $0.4 \mathrm{fm}$. The quality of our projection into the ground state can be evaluated from Figs. 5(a) and 5(b).

For the vector form factors studied here, we used the renormalization factor $Z_{V}$ determined using vector Ward identities in Ref. [43] and summarized in Tables I and II.

The strategy we use in this paper is a perturbative expansion in powers of $\bar{\theta}$ (the expansion is fully performed in Euclidean space). This is justified by the small value of $\bar{\theta}$ estimated from experimental constraints. With this strategy every correlation function $\langle O\rangle_{\bar{\theta}}$, evaluated in a $\bar{\theta}$ vacuum, is determined from a small $-\bar{\theta}$ expansion,

$$
\langle O\rangle_{\bar{\theta}}=\langle O\rangle+i \bar{\theta}\langle O Q\rangle+\mathcal{O}\left(\bar{\theta}^{2}\right),
$$

where $O$ is some multi-local operator, $\bar{\theta}$ is the coefficient for the CP-violating term, and $Q$ is the topological charge. The expectation values on the right-hand side of Eq. (7) are computed on a standard QCD background. This allows us to use lattice QCD gauge configurations without generating new gauges for this specific calculation. We define the correlation functions used to determine the nucleon EDM induced by the $\bar{\theta}$ term in the next section.

The topological charge CP-violating operator that enters the correlation functions must in principle be normalized. We use the gradient flow [17] to define the topological charge which in this way has a finite continuum limit and does not need any additional normalization [17-19]. The reason is that 


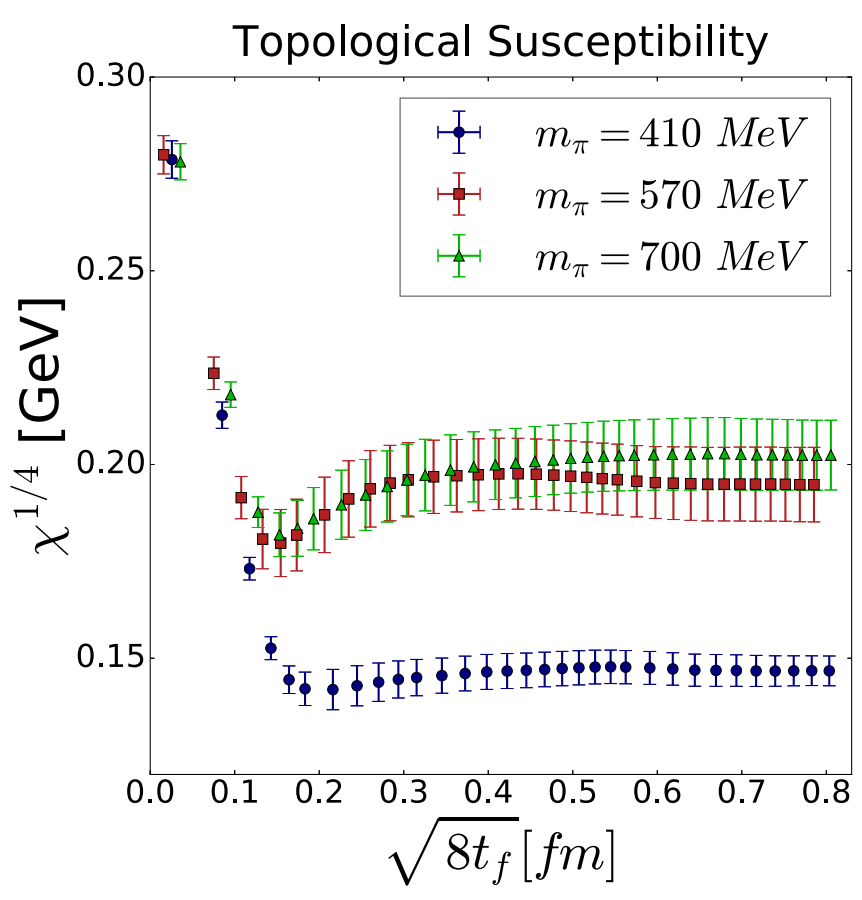

(a)

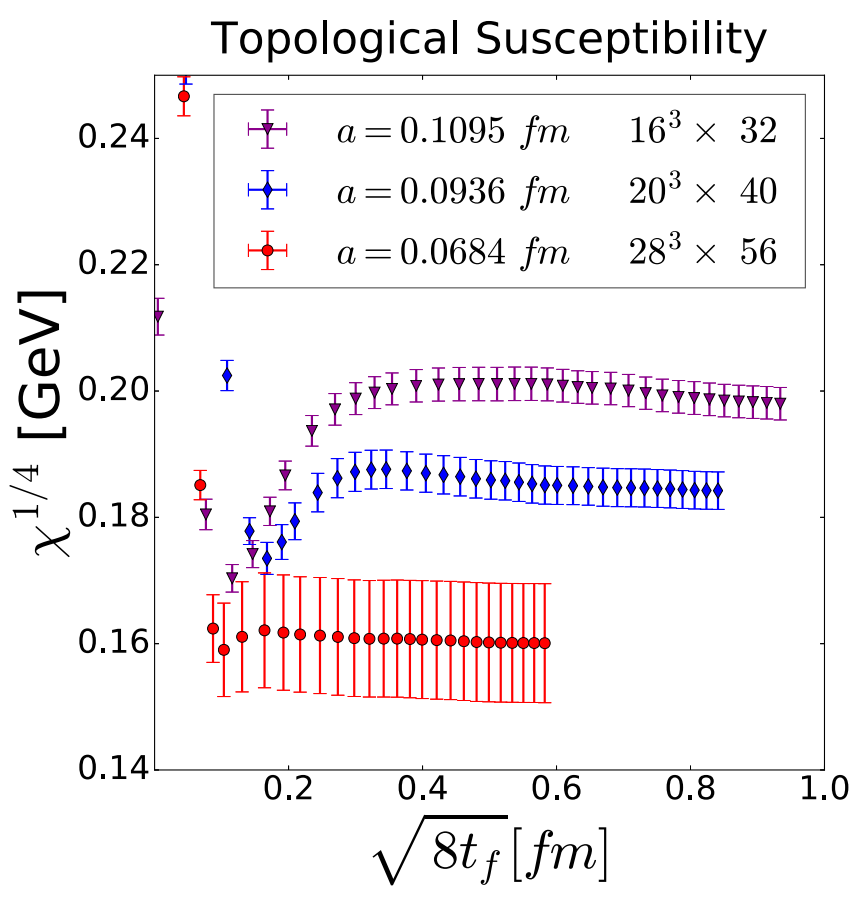

(b)

FIG. 1. The topological susceptibility in $\mathrm{GeV}$ computed for the $\mathrm{M}$ (left) and A ensembles (right), plotted against the flow-time radius $\sqrt{8 t_{f}}$ in $\mathrm{fm}$.

the flowed fields are free from ultraviolet divergences $[17,18]$ for all positive flow times, $t_{f}>0$. Additionally it can be shown that the topological charge defined with the gradient flow is flow-time independent [19] for all positive flow times, $t_{f}>0$, in the continuum limit. Details on how we numerically perform the flowing of the fields have been described in Ref. [11].

\section{TOPOLOGICAL CHARGE AND THE GRADIENT FLOW}

We define the topological charge at finite lattice spacing as

$$
Q\left(t_{f}\right)=a^{4} \sum_{x} q\left(x, t_{f}\right)
$$

where the topological charge density reads

$$
q\left(x, t_{f}\right)=\frac{1}{64 \pi^{2}} \epsilon_{\mu \nu \rho \sigma} G_{\mu \nu}^{a}\left(x, t_{f}\right) G_{\rho \sigma}^{a}\left(x, t_{f}\right),
$$

and $G_{\mu \nu}^{a}\left(x, t_{f}\right)$ is a lattice discretization of the continuum field tensor defined with flowed gauge fields. As a lattice definition for the field tensor, we use the discretization suggested in Ref. [44]. This definition suffers from small discretization effects and, in fact, the corresponding topological susceptibility

$$
\chi\left(t_{f}\right)=\frac{a^{8}}{V} \sum_{x, y}\left\langle q\left(x, t_{f}\right) q\left(y, t_{f}\right)\right\rangle
$$

is flow-time independent starting from a flow-time radius, $\sqrt{8 t_{f}}$, of about $1 \mathrm{fm}$ for all lattice spacings we have investigated. This can be seen in Fig. 1, where we show the flow-time dependence of the topological susceptibility computed for all $\mathrm{M}$ (left) and A ensembles (right). As expected, the region where the susceptibility is independent of the flow time extends toward smaller flow-time values for smaller lattice spacings.

We used the topological charge to perform various checks on the quality of the ensembles. An important check for EDM calculations is to make sure that the ensembles sample the field space in such a way that no spurious $\mathrm{CP}$-violation is induced. In other words we must check the expectation value $\left\langle Q\left(t_{f}\right)\right\rangle=0$ within statistical errors. In Fig. 2 we show $\left\langle Q\left(t_{f}\right)\right\rangle$ evaluated on all our ensembles for various pion masses and lattice spacings. To properly estimate the statistical uncertainties we evaluate the autocorrelation function and the corresponding integrated autocorrelation time, $\tau_{\text {int }}$, as defined in Ref. [45]. For all our ensembles the average topological charge vanishes within statistical errors. In Fig. 3 we show the flow-time dependence of the integrated autocorrelation time for the topological charge. As expected the gradient flow, by smoothing out some of the short-distance fluctuations, allows a better determination of $\tau_{\text {int }}$ that reaches a plateau for $\sqrt{8 t_{f}} \simeq$ $0.2 \mathrm{fm}$ for all our ensembles [46,47].

The integrated autocorrelation time $\tau_{\text {int }}$ we obtain falls within the range $7<\tau_{\text {int }}<35$ for the $\mathrm{M}_{1}$ and $\mathrm{M}_{2}$ ensembles and slightly smaller, $3<\tau_{\text {int }}<10$, for our $\mathrm{M}_{3}$ ensemble. We attribute this behavior with the rather short Markov chain for the $\mathrm{M}_{3}$ ensemble which most likely does not allow a more accurate determination of its $\tau_{\text {int }}$. We also observe from Fig. 3 that $\tau_{\text {int }}$ increases as we decrease the lattice spacing. This is an expected result $[46,48]$ since the tunnelling between different topological sectors becomes increasingly difficult with decreasing lattice spacing, meaning that the 


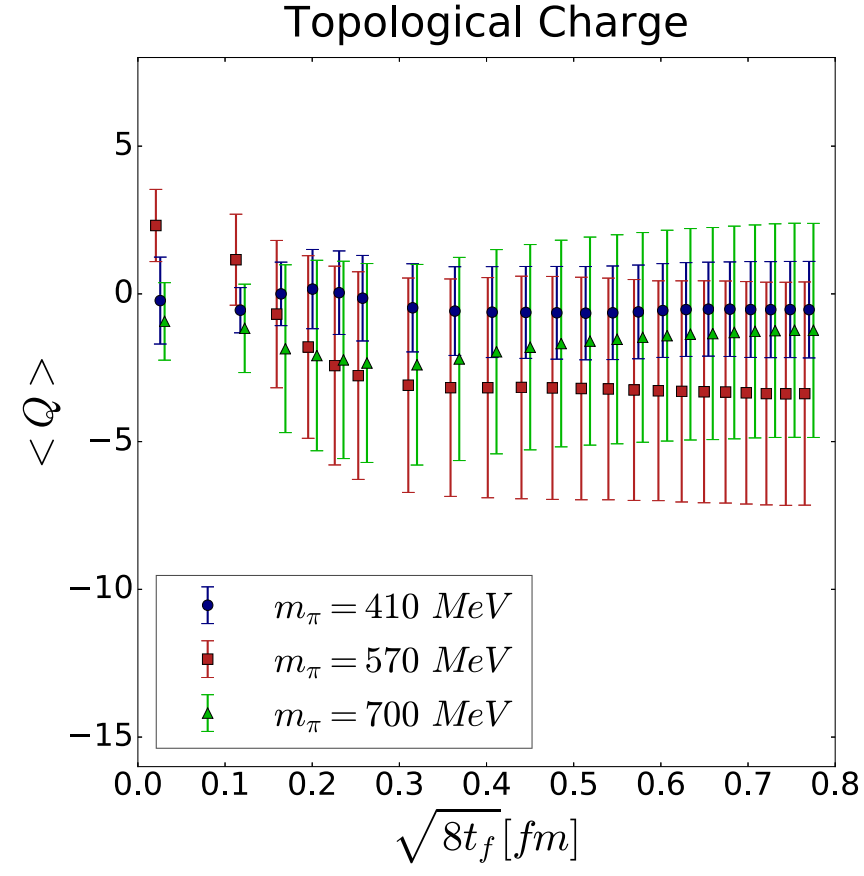

(a)

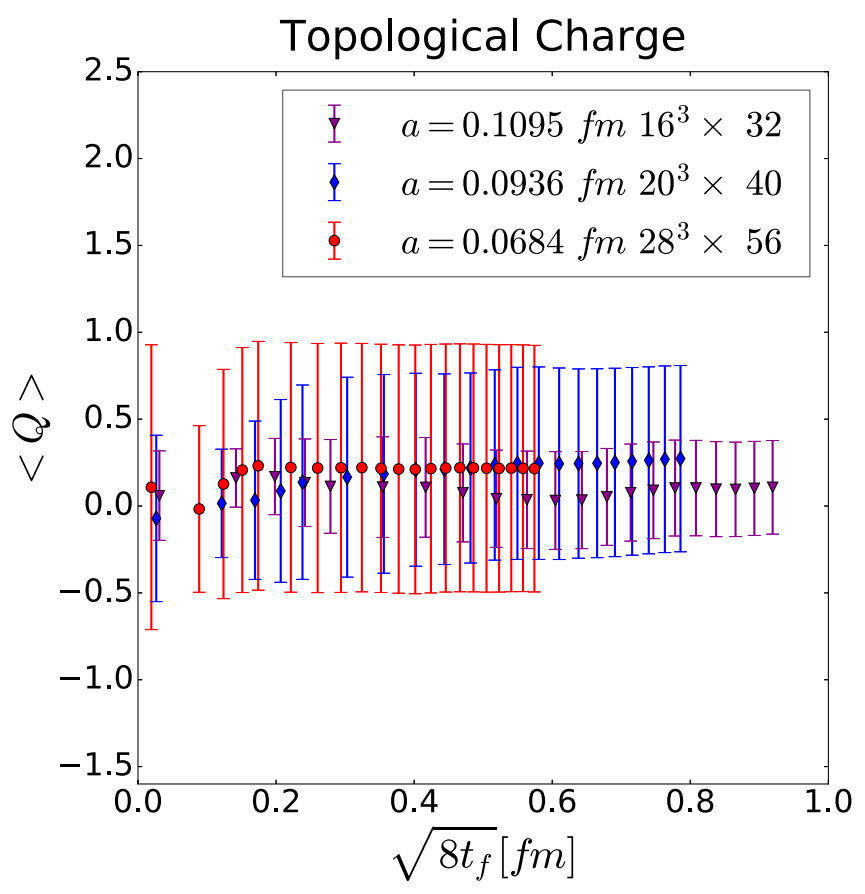

(b)

FIG. 2. Flow-time radius $\sqrt{8 t_{f}}$ dependence of the topological charge $\langle Q\rangle$ for the M (left) and A ensembles (right). The errors are computed using an autocorrelation analysis as described in Ref. [45].

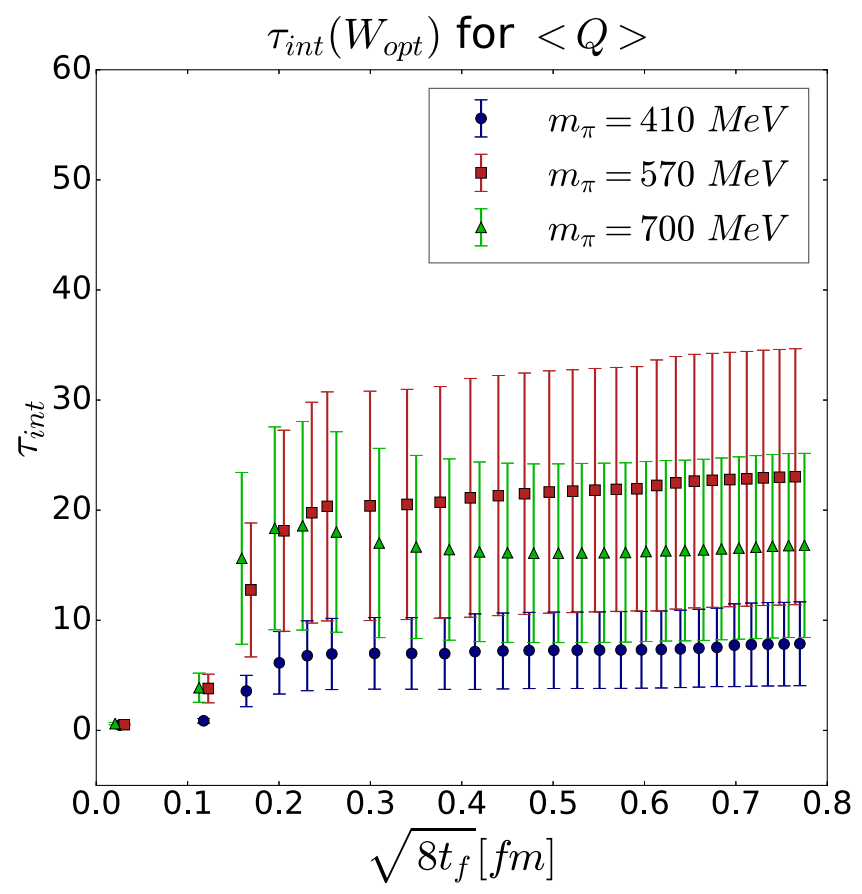

(a)

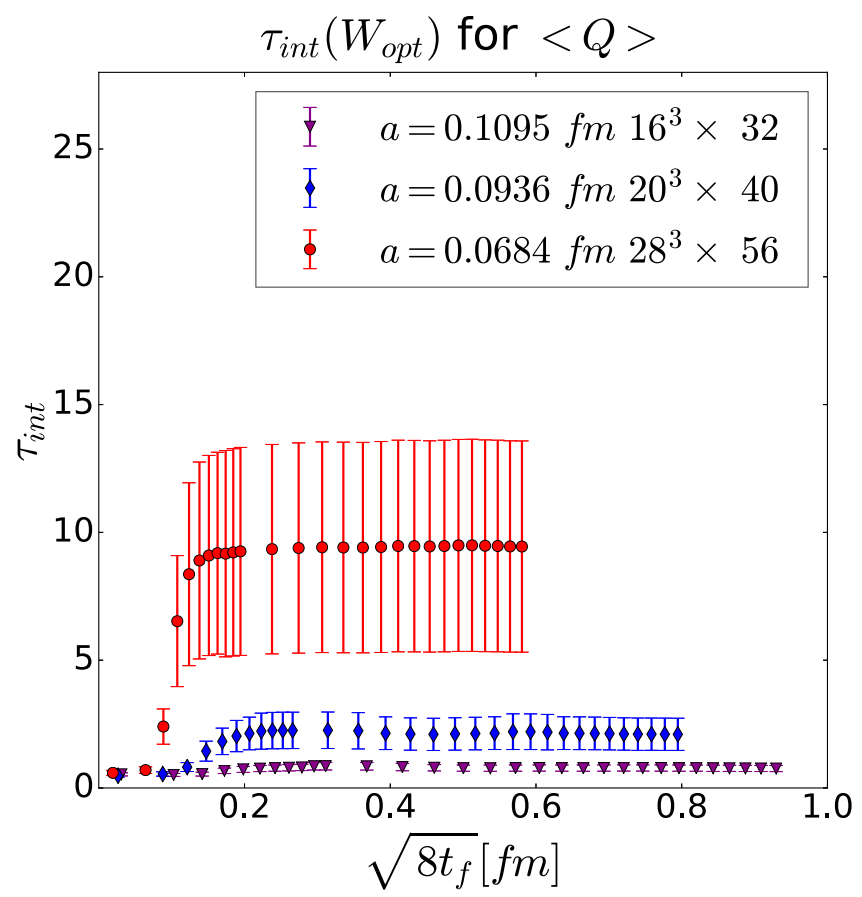

(b)

FIG. 3. Flow-time radius $\sqrt{8 t_{f}}$ dependence of the optimal integrated correlation time $\tau_{\text {int }}$ of the topological charge for the M-(left) and A-(right) ensembles. The error calculation, as well as the optimal autocorrelation length $W_{\text {opt }}$, are computed as described in Ref. [45]. 


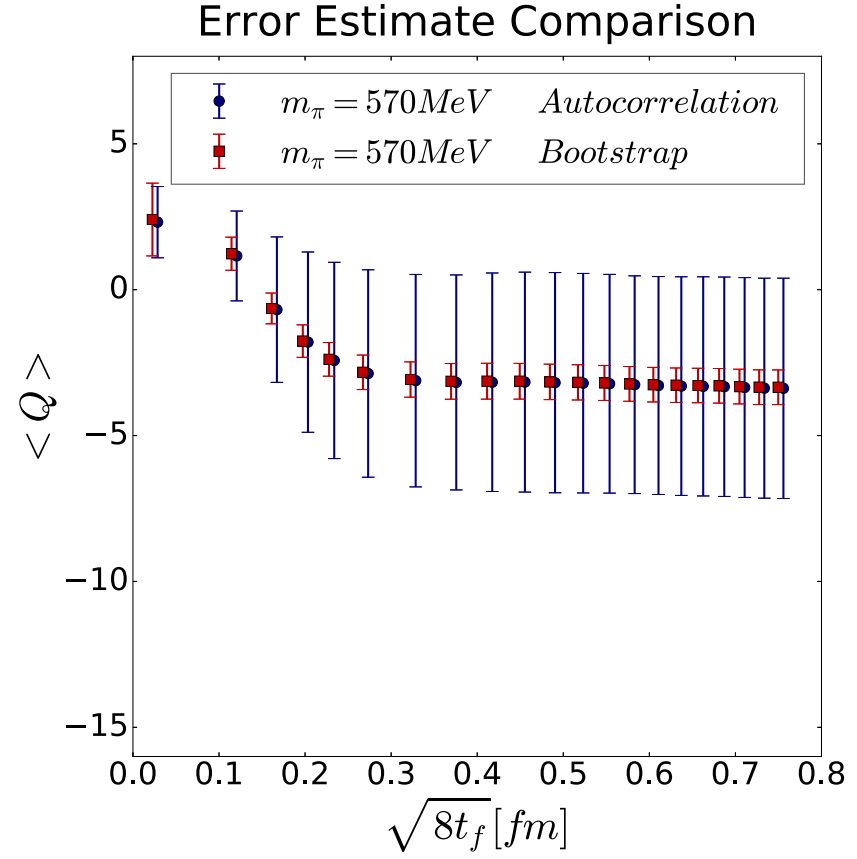

(a)

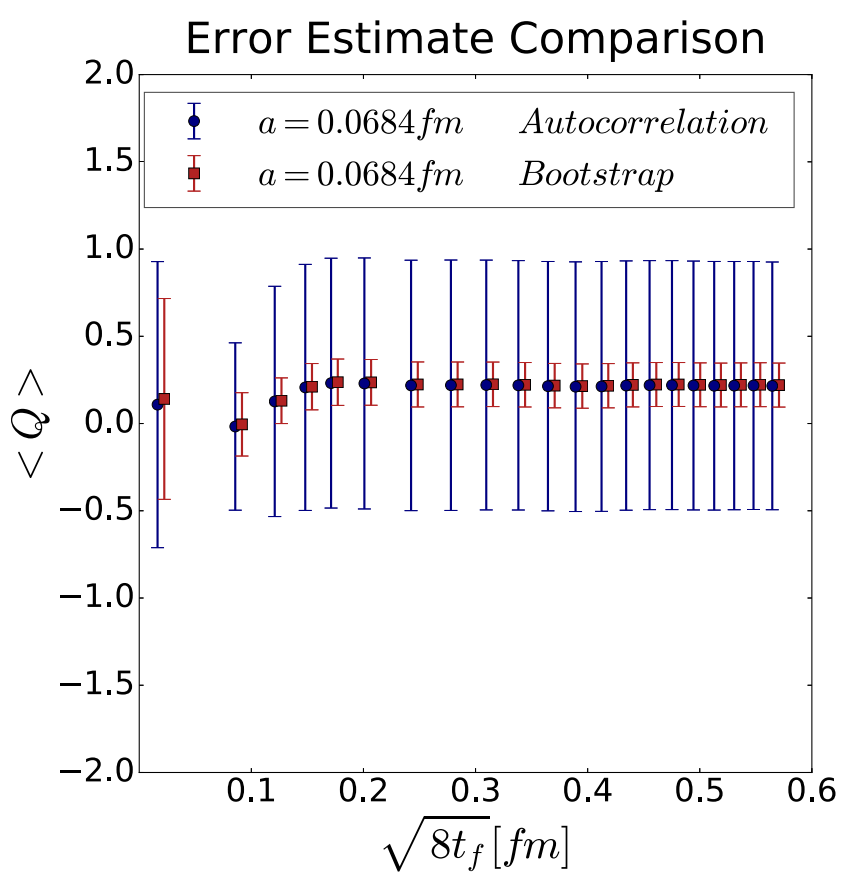

(b)

FIG. 4. Flow-time-radius dependence of the topological charge for the ensemble $\mathrm{M}_{2}$ and $\mathrm{A}_{3}$ with statistical errors computed using the autocorrelation function (blue data points) and a standard bootstrap error estimate (red data points).

sampling of different sectors, which would decrease $\tau_{\text {int }}$, is lessened.

For completeness in Fig. 4 we show the difference of error determination if we were to use a standard resampling technique, such as bootstrap, instead of the error determination using the autocorrelation function. In this case, $\left\langle Q\left(t_{f}\right)\right\rangle \neq 0$ within uncertainties. This demonstrates how a robust uncertainty determination for the topological charge requires both the estimate of the autocorrelation function and its corresponding integrated autocorrelation time.

\section{TWO-POINT CORRELATION FUNCTIONS AND THE NUCLEON MIXING ANGLE}

In this section we analyze the nucleon two-point correlation function used to extract the effective mass, as well as the nucleon mixing angle. The standard two-point correlation function with sink momentum $\boldsymbol{p}^{\prime}$ has the form

$$
G_{2}\left(\boldsymbol{p}^{\prime}, t, \Pi\right)=a^{3} \sum_{\boldsymbol{x}} e^{-i \boldsymbol{p}^{\prime} \cdot \boldsymbol{x}} \operatorname{Tr}\{\Pi\langle\mathcal{N}(\boldsymbol{x}, t) \overline{\mathcal{N}}(\mathbf{0}, 0)\rangle\}
$$

where $\Pi$ is some spin projector, and $\mathcal{N}$ is an interpolating field with the quantum numbers of a nucleon, inserted with a source-sink time separation of $t$. The spectral decomposition for this equation in the limit where $T \gg t \gg 0$, keeping implicit a sum over the polarizations, is

$$
G_{2}\left(\boldsymbol{p}^{\prime}, t, \Pi\right)=\frac{e^{-E_{\beta_{0}} t}}{2 E_{\beta_{0}}} \operatorname{Tr}\left\{\Pi\left\langle\Omega|\mathcal{N}| \beta_{0}\right\rangle\left\langle\beta_{0}|\overline{\mathcal{N}}| \Omega\right\rangle\right\},
$$

where the lowest energy state $\beta_{0}$ for which $\left\langle\Omega|\mathcal{N}| \beta_{0}\right\rangle \neq$ 0 and $\left\langle\beta_{0}|\overline{\mathcal{N}}| \Omega\right\rangle \neq 0$ arises from the approximation ${ }^{1} T \gg$ $t \gg 0$.

The effective mass, which is shown in Fig. 5, is given by the simple log ratio

$$
M_{\mathrm{eff}}\left(\boldsymbol{p}^{\prime}=\mathbf{0}, t, \Pi_{+}\right)=\ln \left[\frac{G_{2}\left(\boldsymbol{p}^{\prime}=\mathbf{0}, t, \Pi_{+}\right)}{G_{2}\left(\boldsymbol{p}^{\prime}=\mathbf{0}, t+1, \Pi_{+}\right)}\right]=m_{\beta_{0}^{+}},
$$

where $\Pi_{+}=\left(I+\gamma_{4}\right) / 2$ is the positive parity projector, $\beta_{0}^{+}$ is the lowest energy positive parity nucleon state, and again, $T \gg t \gg 0$. In Fig. 5(a), we compare our effective mass determinations for the M ensembles to those computed in Ref. [39] and find agreement within statistical errors. As shown in Fig. 5(b), we observe lattice-spacing dependence of the order of $10 \%$ between the finest and coarsest lattices.

The nucleon mixing angle [1], $\alpha_{N}$, can be extracted by utilizing the two-point correlator from Eq. (11), and the $\bar{\theta}$ modified two-point correlator

$$
\begin{aligned}
& G_{2}^{(Q)}\left(\boldsymbol{p}^{\prime}, t, \Pi, t_{f}\right) \\
& \quad=a^{3} \sum_{\boldsymbol{x}} e^{-i \boldsymbol{p}^{\prime} \cdot \boldsymbol{x}} \operatorname{Tr}\left\{\Pi\left\langle\mathcal{N}(\boldsymbol{x}, t) \overline{\mathcal{N}}(\mathbf{0}, 0) Q\left(t_{f}\right)\right\rangle\right\} .
\end{aligned}
$$

\footnotetext{
${ }^{1}$ It is clear from the context when we consider operators as in Eq. (12) or interpolating fields as in Eq. (11).
} 


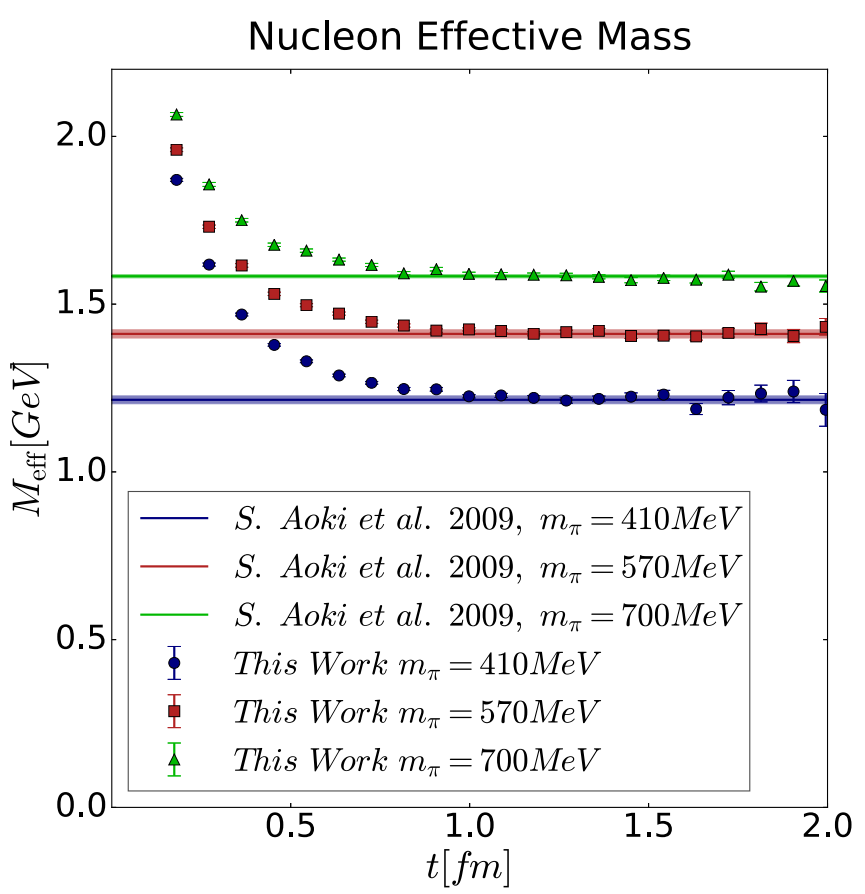

(a)

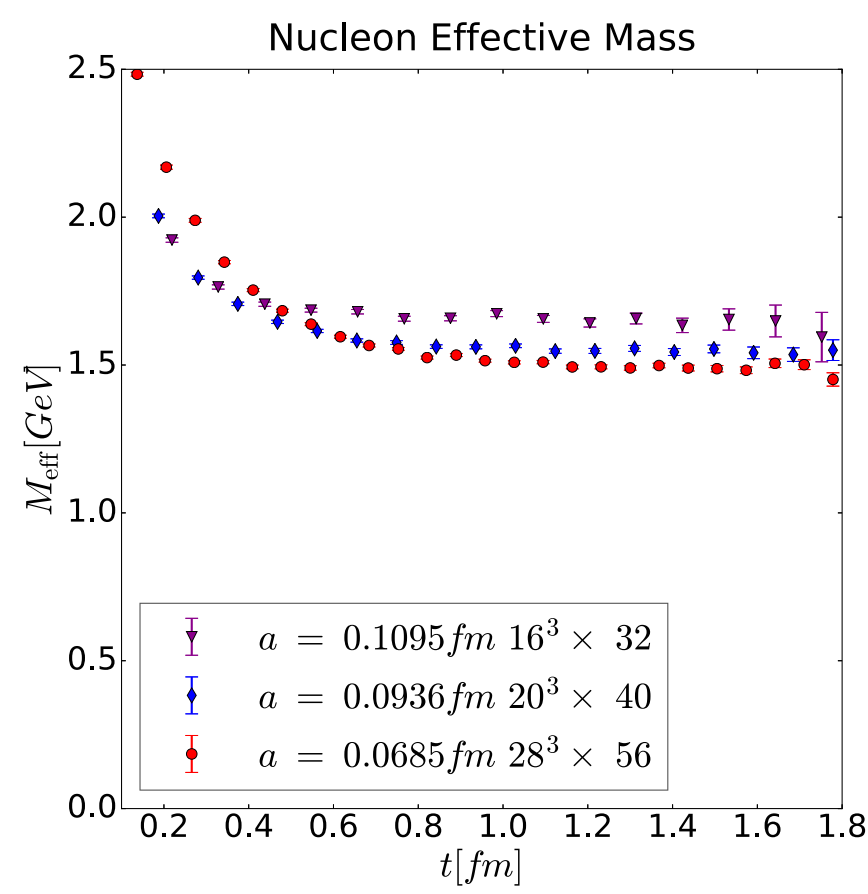

(b)

FIG. 5. Left: $m_{\pi}$ dependence of the effective mass (in $\mathrm{GeV}$ ) defined in Eq. (13) plotted against source-sink separation time $t$. Bands correspond to values quoted in Ref. [39]. Right: lattice-spacing dependence of the effective mass (in GeV) plotted against source-sink separation time $t$.

The mixing angle is defined using the small- $\bar{\theta}$ expansion as

$$
\alpha_{N}=\frac{G_{2}^{(Q)}\left(\boldsymbol{p}^{\prime}=\mathbf{0}, t, \gamma_{5} \Pi_{+}, t_{f}\right)}{G_{2}\left(\boldsymbol{p}^{\prime}=\mathbf{0}, t, \Pi_{+}\right)},
$$

in the region where $t \gg 0$ and $t_{f} \gg 0$.

In Figs. 6(a) and 7(a), we show the dependence of the nuclear mixing angle on the source-sink separation $t$ (in fm) for the $\mathrm{M}$ and $\mathrm{A}$ ensembles, respectively. For the $\mathrm{M}$ ensembles in Figs. 6(a), there is little to no excited-state contamination effects for $t>0.6 \mathrm{fm}$. The results suggest a nontrivial chiral behavior for $\alpha_{N}$. We will discuss this in detail in Sec. VI when we describe our EDM determination. For the lattice-spacing ensembles in Fig. 7(a), we require a minimum source-sink separation of $t \approx a\{5,7,14\} \mathrm{fm}$ (for $a=\{0.1095$, $0.0936,0.0684\} \mathrm{fm}$ ensembles) to obtain a plateau and achieve ground state saturation. The final plateaued quantity for the different A ensembles all lead to the same angle $\alpha_{N}$ and all results are consistent within statistical uncertainties. Similarly, Fig. 7(b) demonstrates that computing $\alpha_{N}$ at $m_{\pi}=700 \mathrm{MeV}$ with different box sizes lead to consistent results. In Table III we summarize the fit ranges and resulting values for $\alpha_{N}$.

The value of the flow-time radius, $\sqrt{8 t_{f}}$, for all these analyses is fixed around $0.5-0.6 \mathrm{fm}$ where the topological charge is least affected by lattice artifacts. Figures 8 (a) and 9(a) show the nucleon mixing angle plotted against the flow time $\sqrt{8 t_{f}}$ at a fixed source-sink separation $t$ for the $\mathrm{M}$ and $\mathrm{A}$ ensembles. For the $\mathrm{M}$ ensembles, we see no flow-time dependence after $\sqrt{8 t_{f}}>0.2-0.3 \mathrm{fm}$, confirming that the results obtained in this region are free from gradient-flow discretization effects. A similar conclusion is reached for the A ensembles.

In Figs. 6(b) we show the integrated autocorrelation time of $\alpha_{N}$ for the $\mathrm{M}$ ensemble results shown in Fig. 6(a). For the $M_{1}$ and $M_{2}$ ensembles a factor of 2-4 increase in autocorrelation as the source-sink separation approaches 0. Fortunately, a minimum source-sink separation of $t \approx 1 \mathrm{fm}$ greatly decreases the autocorrelation correction that we apply in the determination of the nucleon mixing angle $\alpha_{N}$. Most importantly, in comparison to $\langle Q\rangle$ from Fig. 3 (i.e., not in the presence of a nucleon), the autocorrelation effect is dramatically decreased by a factor of at least $\simeq 4$. We attribute this effect to the presence of a fermionic part, $\mathcal{N} \overline{\mathcal{N}}$, in the correlation function. Numerical evidence suggests that the observables considered in this work containing fermion lines, such as $\alpha_{N}$ and the EDM are less coupled to the slow modes contributing to the spectral decomposition of the autocorrelation function [46]. As this effect will be greater when analyzing the EDM (from three-point correlation functions), we resort to our standard bootstrap error propagation technique for the final EDM computation. We checked explicitly that error estimates from a bootstrap and an autocorrelation analysis give consistent results.

\section{A. Improving the nucleon mixing angle}

In this section we describe a method previously explored in Ref. [49], that aims to reduce the statistical uncertainty of the determination of the nucleon mixing angle $\alpha_{N}$. The strategy can be described as an attempt to understand the 


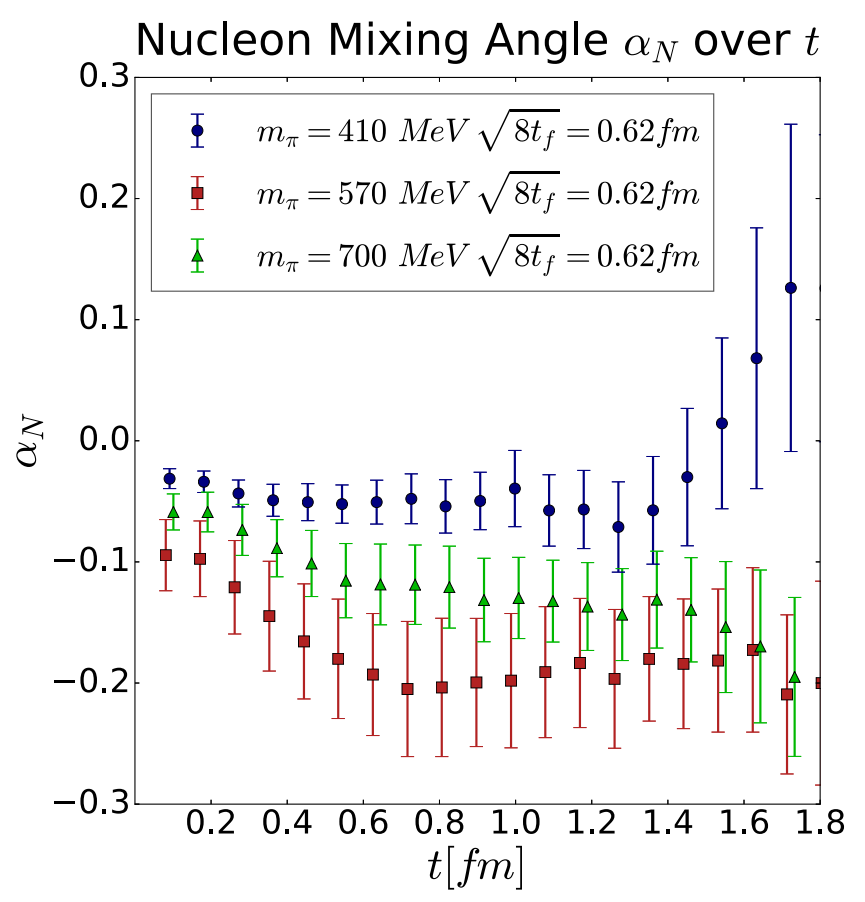

(a)

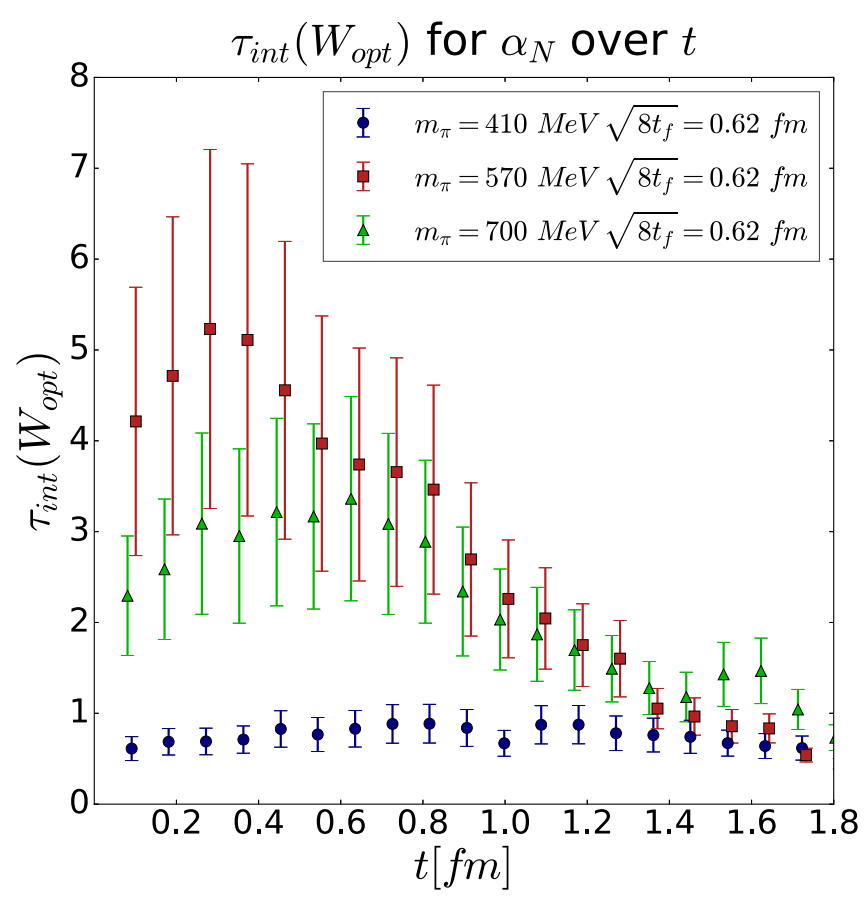

(b)

FIG. 6. Left: The nucleon mixing angle as function of the source-sink separation $t$ at fixed flow time $\sqrt{8 t_{f}}=0.62 \mathrm{fm}$ for different pion masses. Right: Integrated autocorrelation of left plot.

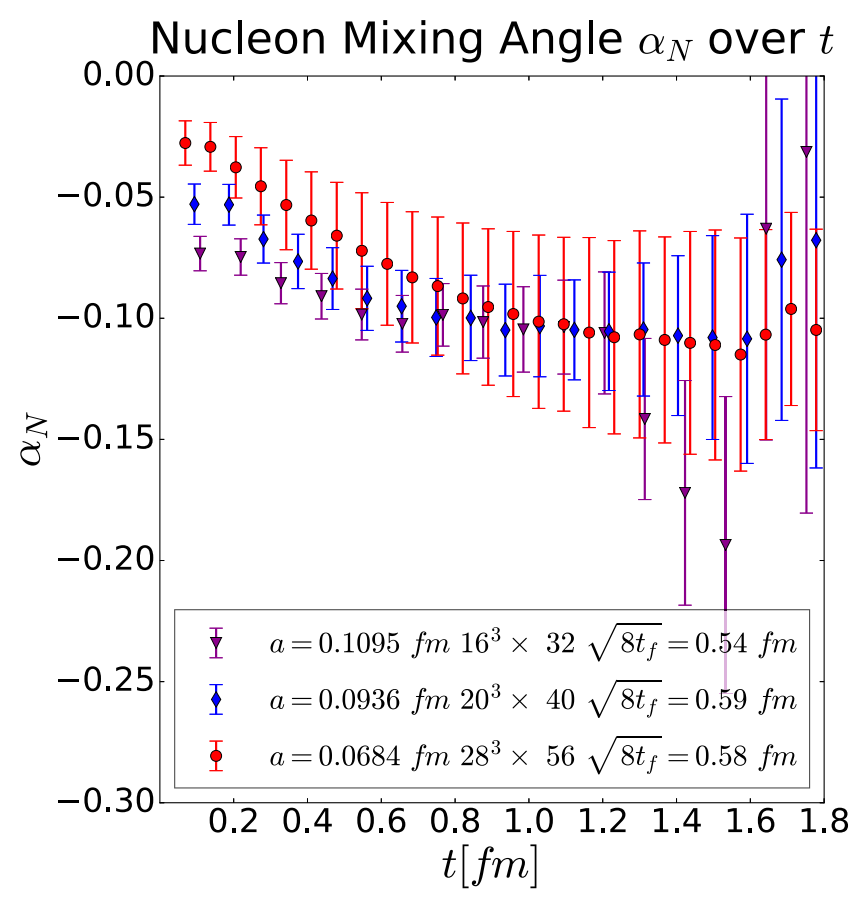

(a)

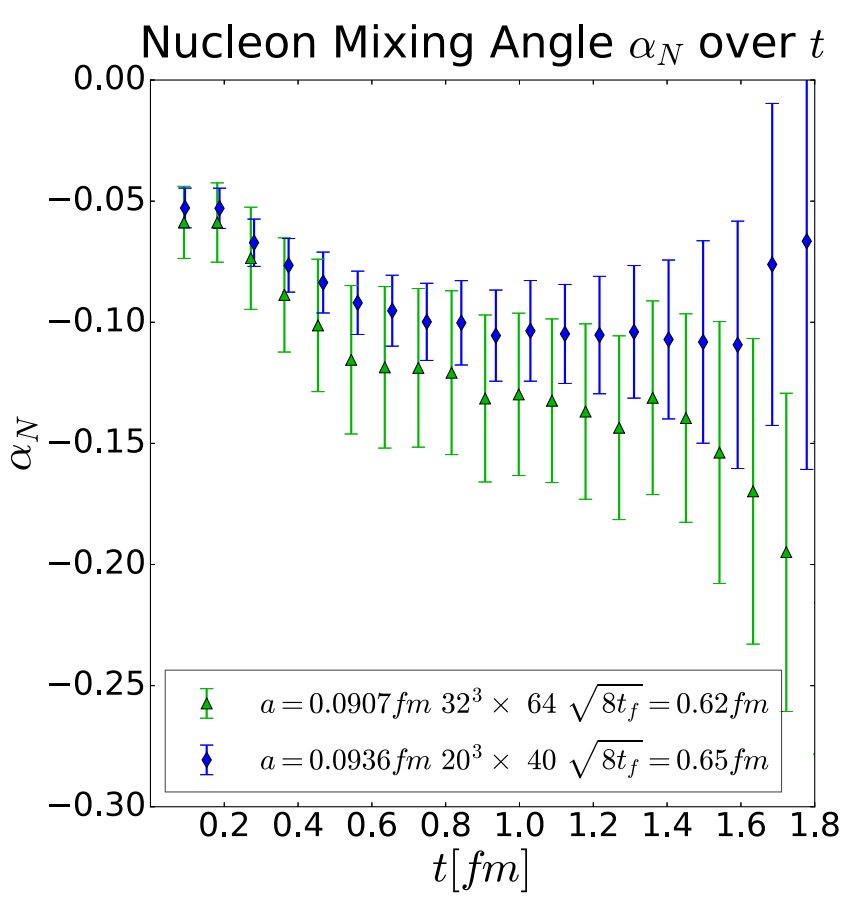

(b)

FIG. 7. Nucleon mixing angle as function of the source-sink separation $t$ at fixed flow time for the A ensembles (left) and box size ensembles (right). 


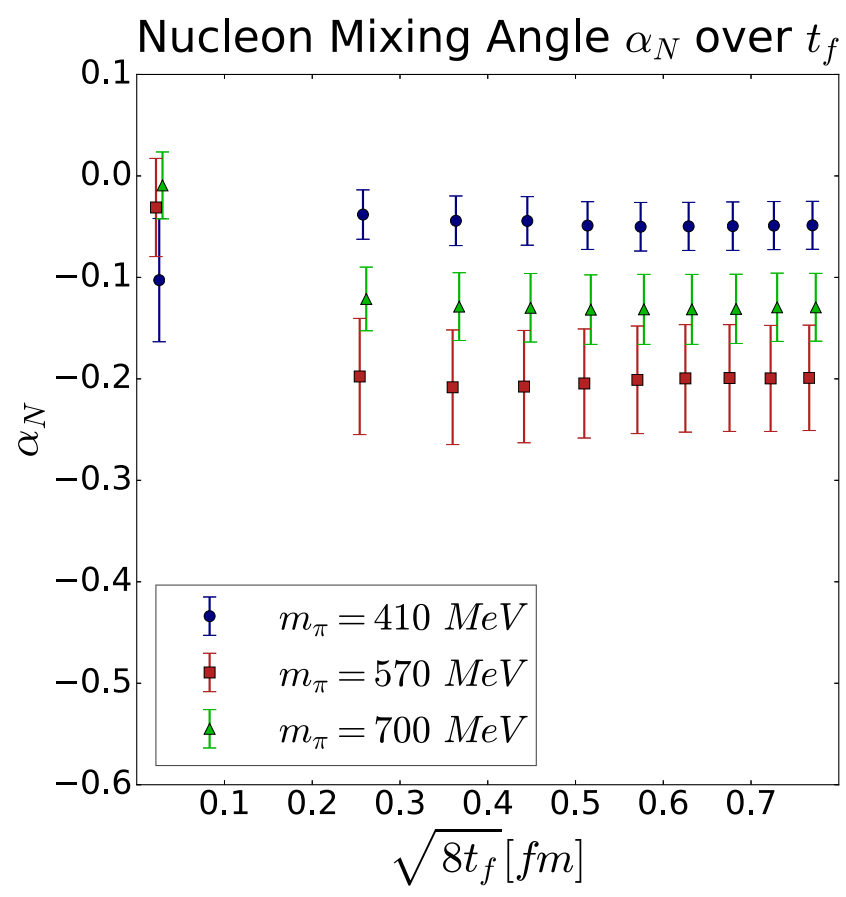

(a)

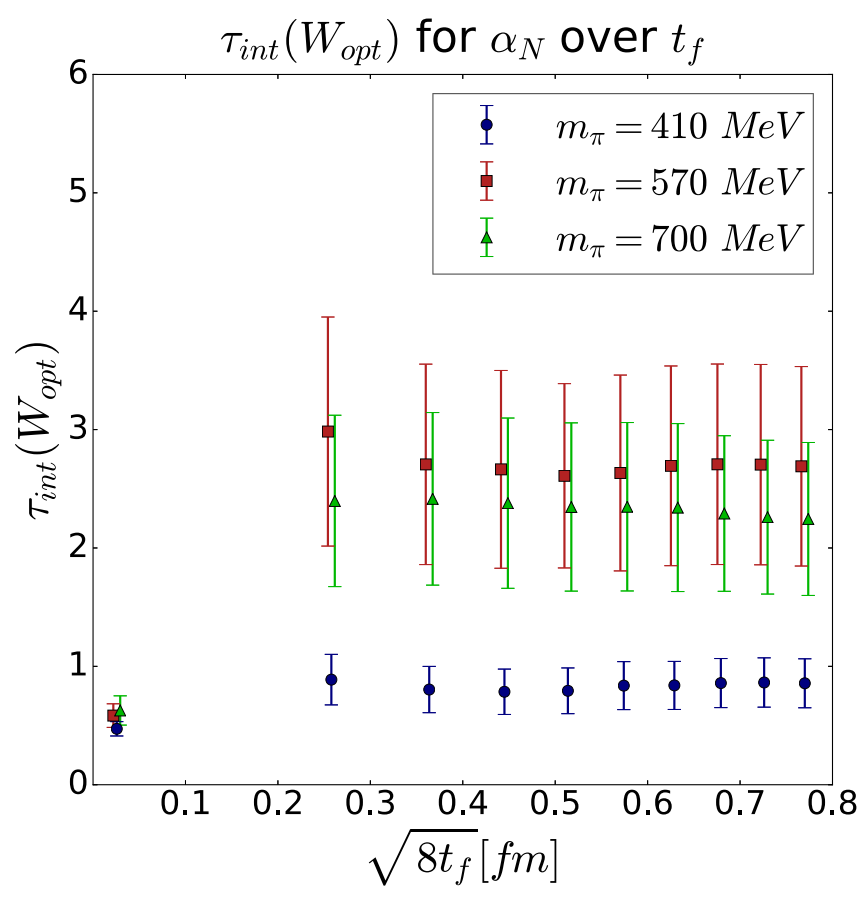

(b)

FIG. 8. Left: The nucleon mixing angle as function of the flow-time radius $\sqrt{8 t_{f}}$, at fixed source-sink separation $t=0.91 \mathrm{fm}$ for different pion masses. Right: Integrated autocorrelation of left plot.

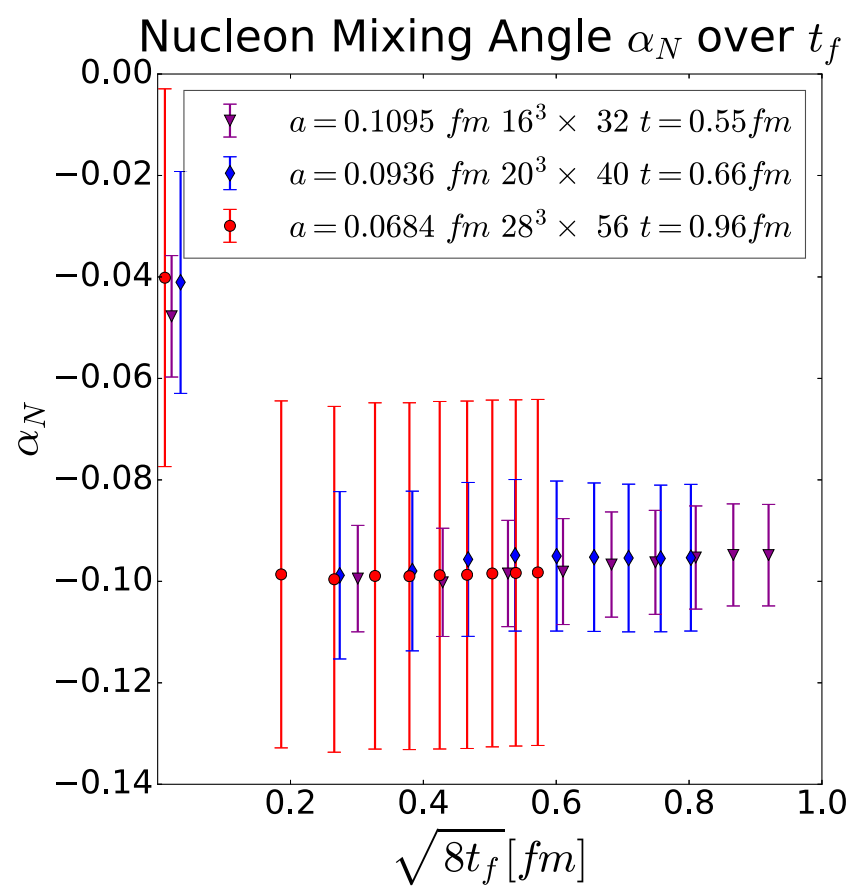

(a)

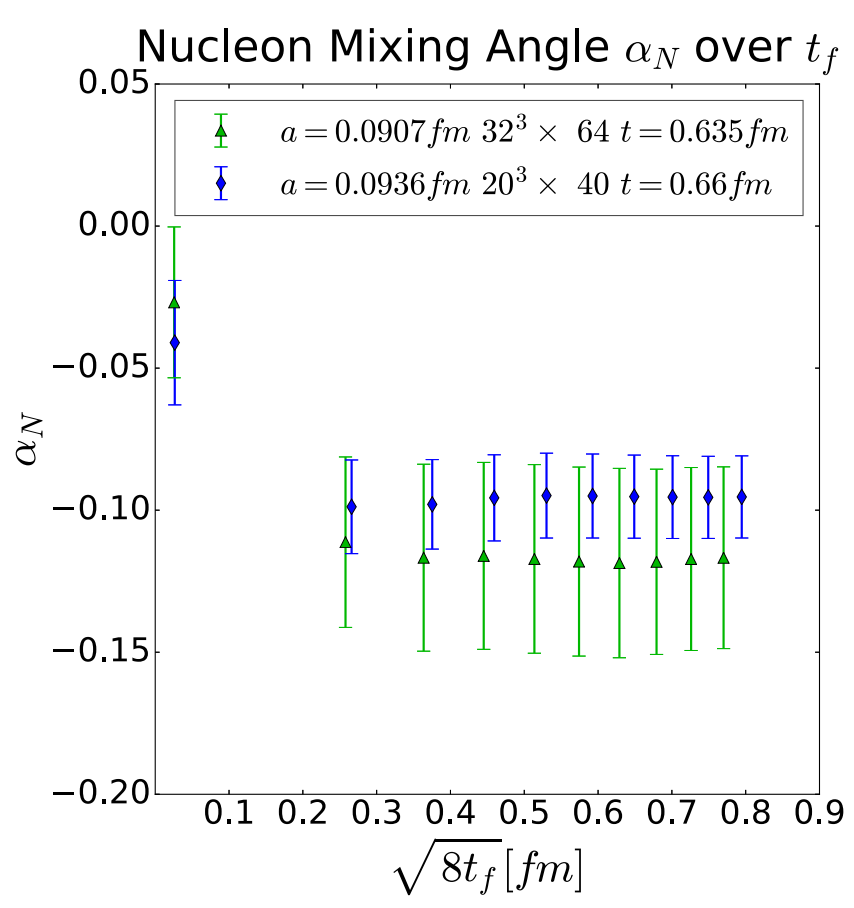

(b)

FIG. 9. Nucleon mixing angle as function of the flow time radius $\sqrt{8 t_{f}}$ at fixed source-sink separation $t$ for various A ensembles (left) and box size ensembles (right). 
TABLE III. Fit ranges $\left[t^{\min }, t^{\max }\right]$ over euclidean source-sink separation $t$ used to extract the nucleon mixing angle $\alpha_{N}$, along with the resulting value.

\begin{tabular}{lcccccc}
\hline \hline Ensemble & $\mathrm{M}_{3}$ & $\mathrm{M}_{2}$ & $\mathrm{M}_{1}$ & $\mathrm{~A}_{1}$ & $\mathrm{~A}_{2}$ & $\mathrm{~A}_{3}$ \\
\hline Fit range & {$[10,20]$} & {$[10,20]$} & {$[10,20]$} & {$[5,11]$} & {$[14,21]$} \\
Fitr [fm] & {$[0.9,1.8]$} & {$[0.9,1.8]$} & {$[0.9,1.8]$} & {$[0.6,1.3]$} & {$[0.7,1.7]$} & {$[0.96,1.43]$} \\
$\alpha_{N}$ & $-0.040(21)$ & $-0.190(27)$ & $-0.142(24)$ & $-0.099(11)$ & $-0.103(10)$ & $-0.105(11)$ \\
\hline \hline
\end{tabular}

space-time region where the overlap between the topological charge density and the fermionic part of the correlation function is maximal. To perform this investigation we define a spatially summed topological charge density,

$$
\begin{gathered}
\bar{Q}\left(\tau_{Q}, t_{f}\right)=a^{3} \sum_{x} q\left(\boldsymbol{x}, \tau_{Q} ; t_{f}\right), \\
Q\left(t_{f}\right)=a \sum_{\tau_{Q}} \bar{Q}\left(\tau_{Q}, t_{f}\right) .
\end{gathered}
$$

We then numerically study the dependence on $\tau_{Q}$ of $\alpha_{N}$ and corroborate our numerical findings with a spectral decomposition of the relevant correlators.

The ratio $\alpha_{N}$ and the modified two-point correlator $G_{2}^{(Q)}$ have the same $\tau_{Q}$ dependence and we therefore focus on the latter. Setting $\boldsymbol{p}^{\prime}=\mathbf{0}$ and omitting it in our expressions, we define

$$
\begin{aligned}
& \Delta_{2}^{(\bar{Q})}\left(t, \Pi, t_{f}, \tau_{Q}\right) \\
& \quad=a^{3} \sum_{\boldsymbol{x}} \operatorname{Tr}\left\{\Pi\left\langle\mathcal{N}(\boldsymbol{x}, t) \bar{Q}\left(\tau_{Q}, t_{f}\right) \overline{\mathcal{N}}(\mathbf{0}, 0)\right\rangle\right\},
\end{aligned}
$$

where the correlator in Eq. (14) can be obtained by summing $\tau_{Q}$ from 0 to the time extent of the lattice $T$,

$$
G_{2}^{(Q)}\left(t, \Pi, t_{f}\right)=a \sum_{\frac{\tau_{Q}}{a}=0}^{T / a} \Delta_{2}^{(\bar{Q})}\left(t, \Pi, t_{f}, \tau_{Q}\right) .
$$

To focus on the region where the signal resides, we sum the spatially summed topological charge density, $\bar{Q}\left(\tau_{Q}, t_{f}\right)$, symmetrically starting from the source location. That is we sum $\tau_{Q}$ starting from 0 (and $T$ ) up to a value $t_{s}$ (and $T-t_{s}$ ).
The goal is to find a summation window $t_{s}$ small enough such that we capture all the signal and avoid the summation of unnecessary "noise." We define the partial summed correlator

$$
\begin{aligned}
\bar{G}_{2}^{(\bar{Q})}\left(t, \Pi, t_{f}, t_{s}\right)= & a \sum_{\frac{\tau_{Q}}{a}=0}^{t_{s} / a}\left[\Delta_{2}^{(\bar{Q})}\left(t, \Pi, t_{f}, \tau_{Q}\right)\right. \\
& \left.+\Delta_{2}^{(\bar{Q})}\left(t, \Pi, t_{f}, T-\tau_{Q}\right)\right],
\end{aligned}
$$

from which, using the periodicity of our lattice, the original correlator in Eq. (14) is obtained as

$$
G_{2}^{(Q)}\left(t, \Pi, t_{f}\right)=\bar{G}_{2}^{(\bar{Q})}\left(t, \Pi, t_{f}, t_{s}=T / 2\right) .
$$

Although there are other choices for the starting point of our summation in $\tau_{Q}$, we only consider starting from $\tau_{Q}=0$. In Appendix A we derive a spectral decomposition for the correlator in Eq. (17). We argue that in the limit $t_{s} \gg t \gg 0$, the partially summed correlator $\bar{G}_{2}^{(\bar{Q})}\left(t, \Pi, t_{f}, t_{s}\right)$ is independent of $t_{s}$ and $t$, up to exponentially suppressed corrections. These corrections seem to be rather small, and in fact our numerical experiments indicate that we can safely stop the summation over $\tau_{Q}$ at $t_{s} \simeq t$. In this way we avoid to sum in the region between $t$ and $T / 2$ where numerically the correlators seem to vanish up to statistical fluctuations.

We first fix the source-sink separation $t$ to a large enough value such that effects from excited states are suppressed. We then study the dependence of $\alpha_{N}$ on the summation window $t_{s}$. In Fig. 10 we show the $t_{s}$ dependence of $\alpha_{N}$, for the M ensembles (left), and A ensembles (middle) and two different physical volumes corresponding to $\mathrm{M}_{1}$ and $\mathrm{A}_{2}$ ensembles

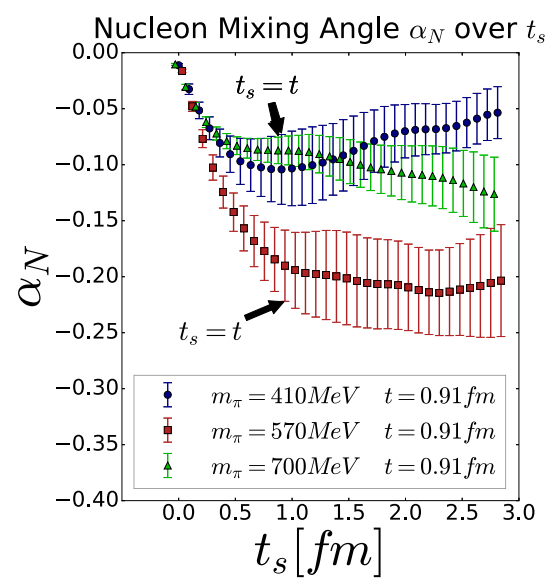

(a)

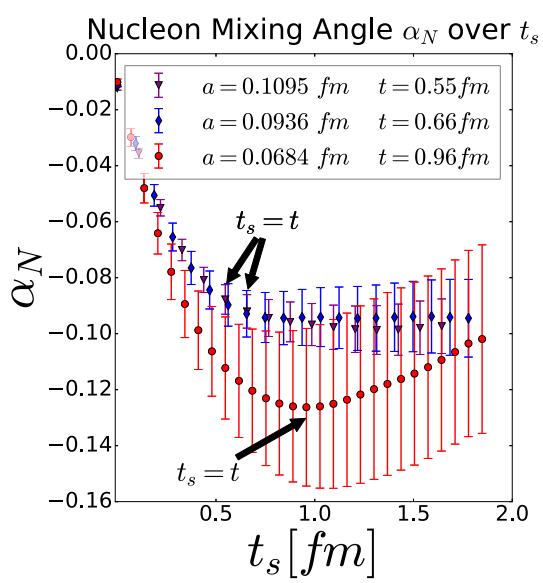

(b)

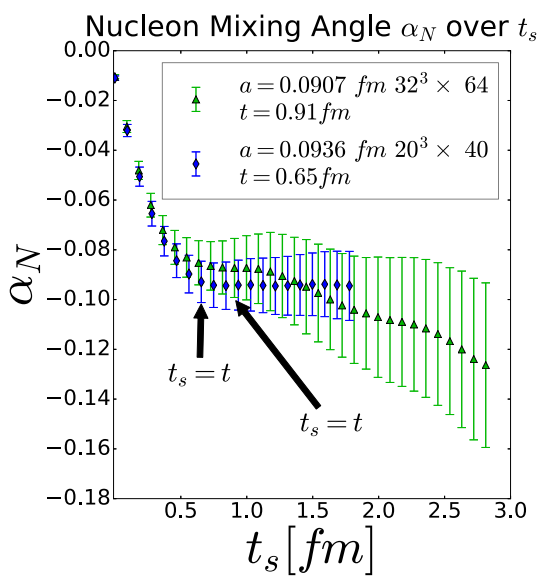

(c)

FIG. 10. M (left), A (middle), and box-size ensembles (right) of the improved nucleon mixing angle $\alpha_{N}$ plotted against the sum parameter $t_{s}$. The final point coincides with the regular nucleon mixing angle from Sec. V. 


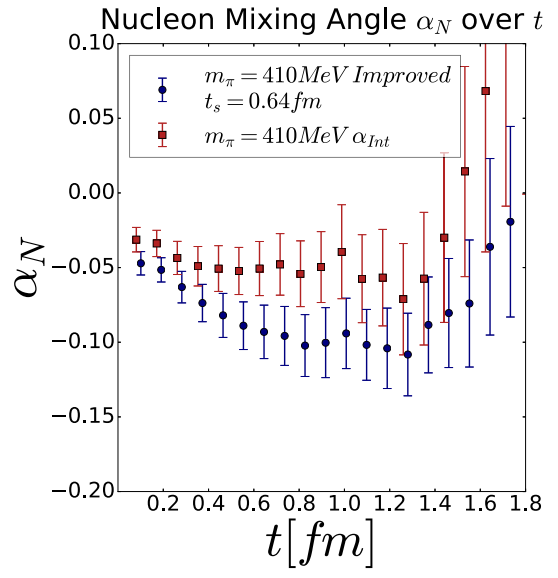

(a)

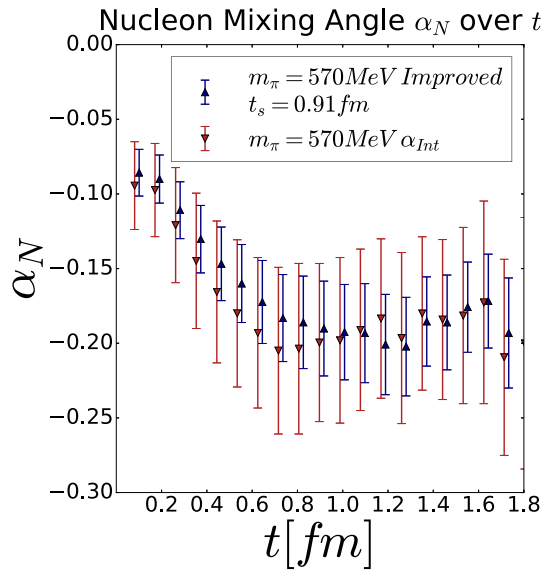

(b)

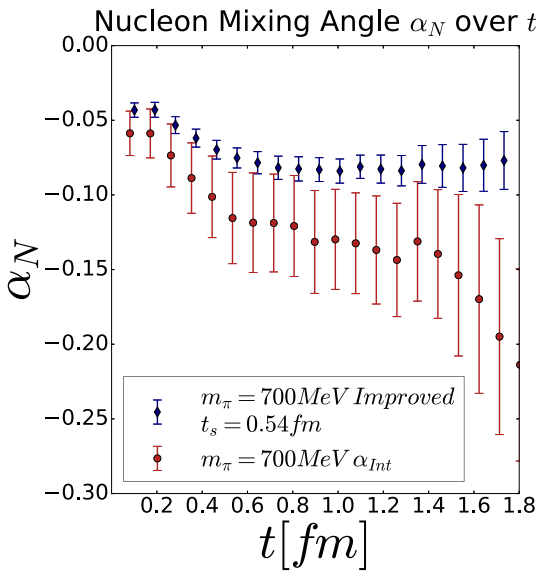

(c)

FIG. 11. $\alpha_{N}$ against $t$ plots for M ensembles, comparing the improved method (blue) to the regular determination described in Sec. V (red).

(right). In all ensembles we observe that $\alpha_{N}$ reaches a plateau when $t_{s} \simeq t$, consistent with the expectation that contributions for $t_{s}>t$ are exponentially suppressed and below our statistical accuracy. We do observe a very small drift of $\alpha_{N}$ for larger values of $t_{s}$ for the ensembles $\mathrm{M}_{1}$, and a smaller drift for the ensemble $\mathrm{M}_{3}$, for small values of $t$. We attribute this to statistical fluctuations that could arise from small local parity-violating effects induced by nonvanishing matrix elements of $\bar{Q}\left(\tau_{Q}, t_{f}\right)$ between two states of the same parity, $\left\langle\beta\left|\bar{Q}\left(\tau_{Q}, t_{f}\right)\right| \beta\right\rangle \neq 0$. These local fluctuations are averaged out when the charge density is summed over the whole spacetime volume as shown in Fig. 2. Nevertheless, all values of $\alpha_{N}$ determined with the improved method are statistically compatible with the results obtained with the standard analysis.

To compare the improved extraction of $\alpha_{N}$ to the standard determination described in Sec. V, we show in Figs. 11 and 12 the standard and improved determination of $\alpha_{N}$ as a function of the Euclidean source-sink separation $t$. The values of $t_{s}$ considered are summarized in Table IV. We observe a signalto-noise improvement in all our ensembles, up to a factor 2 , with the most significant observed in the ensembles $\mathbf{M}_{1}$,
$\mathrm{M}_{2}$, and $\mathrm{A}_{3}$. We observe the largest discrepancy between the improved and unimproved methods, of the order of $2.3 \sigma$, in the $\mathrm{M}_{1}$ and $\mathrm{M}_{3}$ ensembles. We attribute this discrepancy to standard statistical fluctuations of the gauge fields. A summary of the fit ranges and results for the improved nucleon mixing angle is given in Table IV, where for comparison we added the values of $\alpha_{N}$ determined in the standard way.

\section{ELECTRIC DIPOLE MOMENT RESULTS}

The neutron $(n)$ and proton $(p)$ EDMs, $d_{p / n}$, can be extracted from the CP-odd electric dipole form factor ${ }^{2}$

$$
\frac{F_{3}^{p / n}\left(Q^{2}\right)}{2 M_{N}} \stackrel{Q^{2} \ll m_{\pi}^{2}}{\longrightarrow} d_{p / n}-S_{p / n} Q^{2}+O\left(Q^{4}\right),
$$

\footnotetext{
${ }^{2}$ The general form not requiring $Q^{2} \ll m_{\pi}^{2}$, is given in Eq. (58) and discussed in detail in Secs. VIB and VIC. We performed the same analysis with the fit function in Eq. (58) and found insignificant changes to the EDM results (see Secs. VIB and VIC).
}

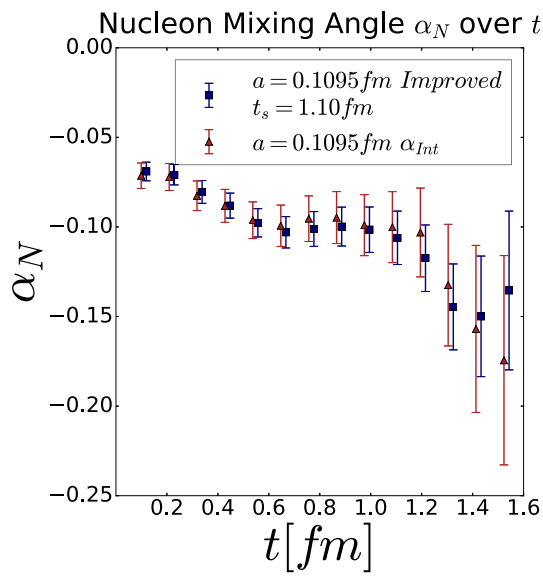

(a)

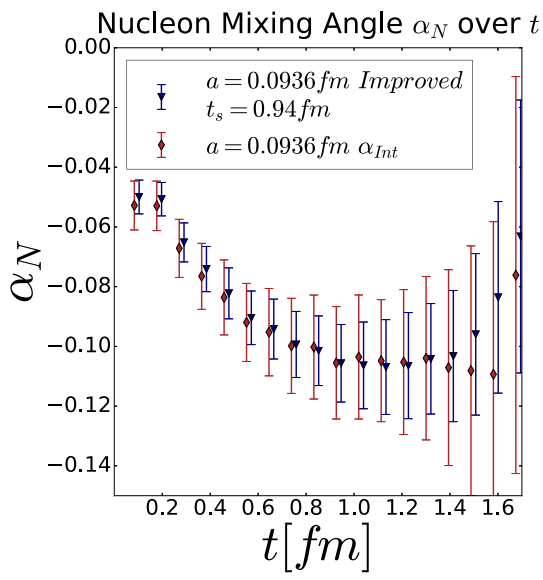

(b)

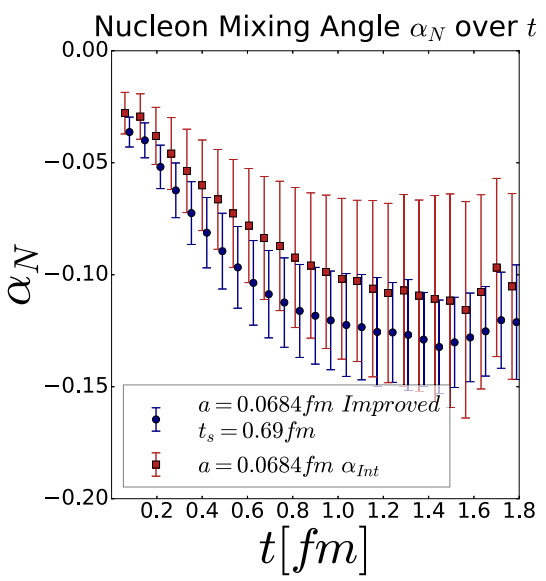

(c)

FIG. 12. $\alpha_{N}$ against $t$ plots for A ensembles, comparing the improved method (blue) to the regular determination described in Sec. V (red). 
which requires a lattice QCD computation of $F_{3}\left(Q^{2}\right)$. The variable $Q^{2}$ in this case refers to the momentum transfer and should not be confused with the topological charge. The small $\bar{\theta}$ expansion provides us a way of accessing $F_{3}$ from threepoint correlation functions without the need for generating new gauge configurations at finite $\bar{\theta}$ and without relying on a problematic analytical continuation to imaginary $\bar{\theta}$. To access $F_{3}\left(Q^{2}\right)$, we calculate the following three-point correlation functions with and without the insertion of the topological charge, respectively,

$$
\begin{aligned}
G_{3}^{(Q)}\left(\boldsymbol{p}^{\prime}, t, \boldsymbol{q}, \tau, \Pi, \gamma_{\mu}, t_{f}\right) & =a^{6} \sum_{\boldsymbol{x}, \boldsymbol{y}} e^{-i\left(\boldsymbol{p}^{\prime} \cdot \boldsymbol{x}-\boldsymbol{q} \cdot \boldsymbol{y}\right)} \operatorname{Tr}\left\{\Pi\left\langle\mathcal{N}(\boldsymbol{x}, t) \mathcal{J}_{\mu}(\boldsymbol{y}, \tau) \overline{\mathcal{N}}(\mathbf{0}, 0) Q\left(t_{f}\right)\right\rangle\right\} \\
G_{3}\left(\boldsymbol{p}^{\prime}, t, \boldsymbol{q}, \tau, \Pi, \gamma_{\mu}\right) & =a^{6} \sum_{\boldsymbol{x}, \boldsymbol{y}} e^{-i\left(\boldsymbol{p}^{\prime} \cdot \boldsymbol{x}-\boldsymbol{q} \cdot \boldsymbol{y}\right)} \operatorname{Tr}\left\{\Pi\left\langle\mathcal{N}(\boldsymbol{x}, t) \mathcal{J}_{\mu}(\boldsymbol{y}, \tau) \overline{\mathcal{N}}(\mathbf{0}, 0)\right\rangle\right\}
\end{aligned}
$$

where the electromagnetic current in terms of the quark currents is given by

$$
\mathcal{J}_{\mu}(\boldsymbol{y}, \tau)=\frac{4}{3} \bar{u}(\boldsymbol{y}, \tau) \gamma_{\mu} u(\boldsymbol{y}, \tau)-\frac{1}{3} \bar{d}(\boldsymbol{y}, \tau) \gamma_{\mu} d(\boldsymbol{y}, \tau)
$$

and $\mathcal{N}$ denotes standard proton or neutron interpolating fields.

Once the three-point correlation functions are computed, we remove the leading Euclidean time dependence and nucleon-to-vacuum amplitude contributions via the ratios:

$$
\begin{aligned}
& R\left(\boldsymbol{p}^{\prime}, t, \boldsymbol{q}, \tau, \Pi, \gamma_{\mu}\right) \\
& =\frac{G_{3}\left(\boldsymbol{p}^{\prime}, t, \boldsymbol{q}, \tau, \Pi, \gamma_{\mu}\right)}{G_{2}\left(\boldsymbol{p}^{\prime}, t, \Pi_{+}\right)} K\left(\boldsymbol{p}^{\prime}, t, \boldsymbol{q}, \tau\right), \\
& R^{(Q)}\left(\boldsymbol{p}^{\prime}, t, \boldsymbol{q}, \tau, \Pi, \gamma_{\mu}, t_{f}\right) \\
& \quad=\frac{G_{3}^{(Q)}\left(\boldsymbol{p}^{\prime}, t, \boldsymbol{q}, \tau, \Pi, \gamma_{\mu}, t_{f}\right)}{G_{2}\left(\boldsymbol{p}^{\prime}, t, \Pi_{+}\right)} K\left(\boldsymbol{p}^{\prime}, t, \boldsymbol{q}, \tau\right),
\end{aligned}
$$

TABLE IV. The selected starting value $t_{s}^{\min }$ for the fit ranges $\left[t_{s}^{\min }, T / 2\right]$ for the summed $\bar{Q}$, the Euclidean source-sink separation fit range $\left[t^{\mathrm{min}}, t^{\mathrm{max}}\right]$ and the resulting nucleon mixing angle $\alpha_{N}$ from the selected parameters. A comparison between computing $\alpha_{N}$ from $t_{s}^{\text {min }}$ and fitting from $t_{s}^{\text {min }}$ onwards showed a negligible difference on all ensembles. We add the values determined in a standard way for comparison.

\begin{tabular}{lccc}
\hline \hline Ensemble & $\mathrm{M}_{3}$ & $\mathrm{M}_{2}$ & $\mathrm{M}_{1}$ \\
\hline$t_{s}^{\min } / a$ & 7 & 10 & 6 \\
$t_{s}^{\min }[\mathrm{fm}]$ & 0.63 & 0.9 & 0.54 \\
$t / a$ fit range & {$[10,20]$} & {$[10,20]$} & {$[10,20]$} \\
$t$ [fm] fit range & {$[0.9,1.8]$} & {$[0.9,1.8]$} & {$[0.9,1.8]$} \\
$\alpha_{N}$ improved & $-0.098(13)$ & $-0.201(17)$ & $-0.0822(97)$ \\
$\alpha_{N}$ standard & $-0.040(21)$ & $-0.190(27)$ & $-0.142(24)$ \\
& & & \\
Ensemble & $\mathrm{A}_{3}$ & $\mathrm{~A}_{2}$ & $\mathrm{~A}_{1}$ \\
\hline$t_{s}^{\min } / a$ & 10 & 10 & 10 \\
$t_{s}^{\min }[$ fm] & 1.21 & 0.98 & 0.69 \\
$t / a$ fit range & {$[5,11]$} & {$[7,17]$} & {$[14,21]$} \\
$t$ [fm] fit range & {$[0.61,1.34]$} & {$[0.69,1.67]$} & {$[0.96,1.44]$} \\
$\alpha_{N}$ improved & $-0.1016(89)$ & $-0.1012(75)$ & $-0.1212(67)$ \\
$\alpha_{N}$ standard & $-0.099(11)$ & $-0.103(10)$ & $-0.105(11)$ \\
\hline \hline
\end{tabular}

where we have implicitly defined ratios for the proton and the neutron. We define the square-root factor as

$$
\begin{aligned}
& K\left(\boldsymbol{p}^{\prime}, t, \boldsymbol{p}, \tau\right) \\
& \quad \equiv \sqrt{\frac{G_{2}\left(\boldsymbol{p}^{\prime}, \tau, \Pi_{+}\right) G_{2}\left(\boldsymbol{p}^{\prime}, t, \Pi_{+}\right) G_{2}\left(\boldsymbol{p}, t-\tau, \Pi_{+}\right)}{G_{2}\left(\boldsymbol{p}, \tau, \Pi_{+}\right) G_{2}\left(\boldsymbol{p}, t, \Pi_{+}\right) G_{2}\left(\boldsymbol{p}^{\prime}, t-\tau, \Pi_{+}\right)}} .
\end{aligned}
$$

The spectral decomposition of the ratio function $R$ in Eq. (24), in the limit $T \gg t \gg 0$, reads

$$
\begin{aligned}
& R\left(\boldsymbol{p}^{\prime}, t, \boldsymbol{q}, \tau, \Pi, \gamma_{\mu}\right) \\
& \quad=A\left(E_{\boldsymbol{p}^{\prime}}, E_{\boldsymbol{p}}\right) \operatorname{Tr}\left\{\Pi\left(-i \not p^{\prime}+m\right) \Gamma_{\mu}\left(Q^{2}\right)(-i \not p+m)\right\},
\end{aligned}
$$

where the vector form factor contains all terms allowed by the symmetries of the theory

$$
\Gamma_{\mu}\left(Q^{2}\right)=\gamma_{\mu} F_{1}\left(Q^{2}\right)+\frac{\sigma_{\mu \nu} q_{\nu}}{2 m} F_{2}\left(Q^{2}\right) .
$$

For completeness the expression of $A\left(E_{p^{\prime}}, E_{p}\right)$ reads

$$
A\left(E_{\boldsymbol{p}^{\prime}}, E_{\boldsymbol{p}}\right)=\frac{1}{4 \sqrt{E_{\boldsymbol{p}^{\prime}} E_{\boldsymbol{p}}\left(E_{\boldsymbol{p}^{\prime}}+m\right)\left(E_{\boldsymbol{p}}+m\right)}} .
$$

The data that we computed coming from the fixed-sink method is such that $\boldsymbol{p}^{\prime}=\mathbf{0}$ (which implies $\boldsymbol{q}=-\boldsymbol{p}$ ), simplifying Eq. (26) to

$$
\begin{aligned}
& R\left(\mathbf{0}, t, \boldsymbol{q}, \tau, \Pi, \gamma_{\mu}\right) \\
& \quad=2 m A\left(m, E_{\boldsymbol{p}}\right) \operatorname{Tr}\left\{\Pi \Pi_{+} \Gamma_{\mu}\left(Q^{2}\right)(-i \not p+m)\right\} .
\end{aligned}
$$

The analogous modified ratio function with the insertion of the topological charge $R^{(Q)}$ in Eq. (24) has, retaining only the ground state contribution, the following spectral decomposition at leading order in $\bar{\theta}$ :

$$
\begin{aligned}
& R^{(Q)}\left(\mathbf{0}, t, \boldsymbol{q}, \tau, \Pi, \gamma_{\mu}, t_{f}\right) \\
& =2 m A\left(m, E_{\boldsymbol{p}}\right)\left[\alpha_{N} 2 m \operatorname{Tr}\left\{\Pi \Pi_{+} \widetilde{\Gamma}_{\mu}\left(Q^{2}\right) \gamma_{5}\right\}\right. \\
& \quad+\alpha_{N} \operatorname{Tr}\left\{\Pi \gamma_{5} \widetilde{\Gamma}_{\mu}\left(Q^{2}\right)(-i \not p+m)\right\} \\
& \left.\quad+\operatorname{Tr}\left\{\Pi \Pi_{+} \frac{\sigma_{\mu \nu} \gamma_{5} q_{v}}{2 m} \widetilde{F}_{3}\left(Q^{3}\right)(-i \not p+m)\right\}\right],
\end{aligned}
$$

where

$$
\widetilde{\Gamma}_{\mu}\left(Q^{2}\right)=\gamma_{\mu} F_{1}\left(Q^{2}\right)+\frac{\sigma_{\mu \nu} q_{\nu}}{2 m} \widetilde{F}_{2}\left(Q^{2}\right)
$$




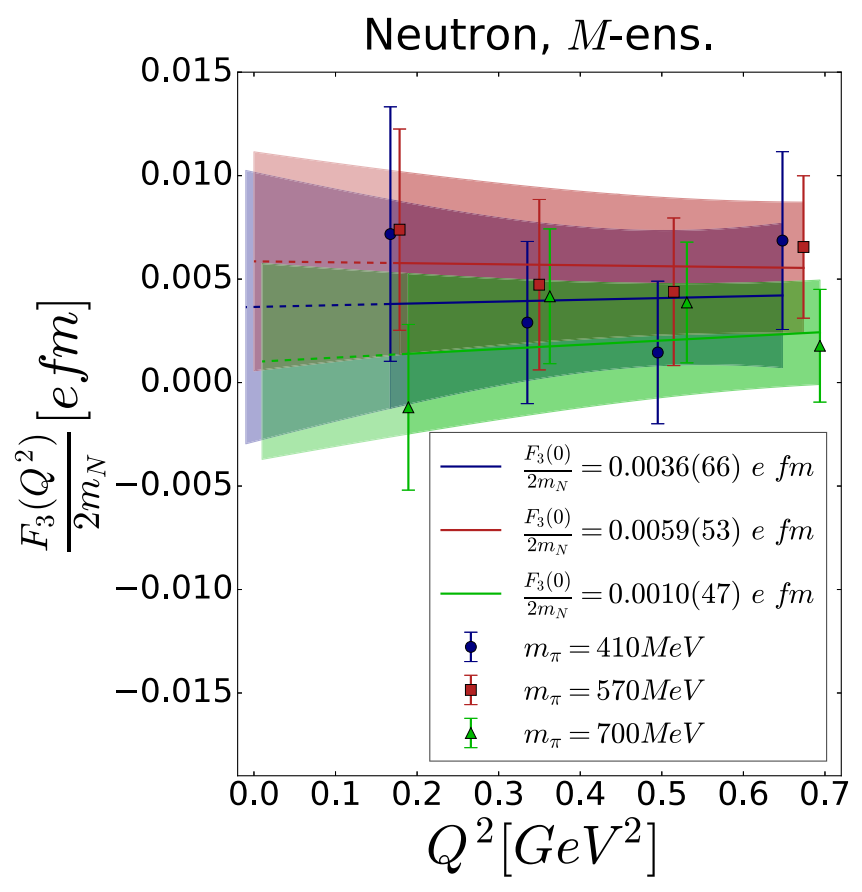

(a)

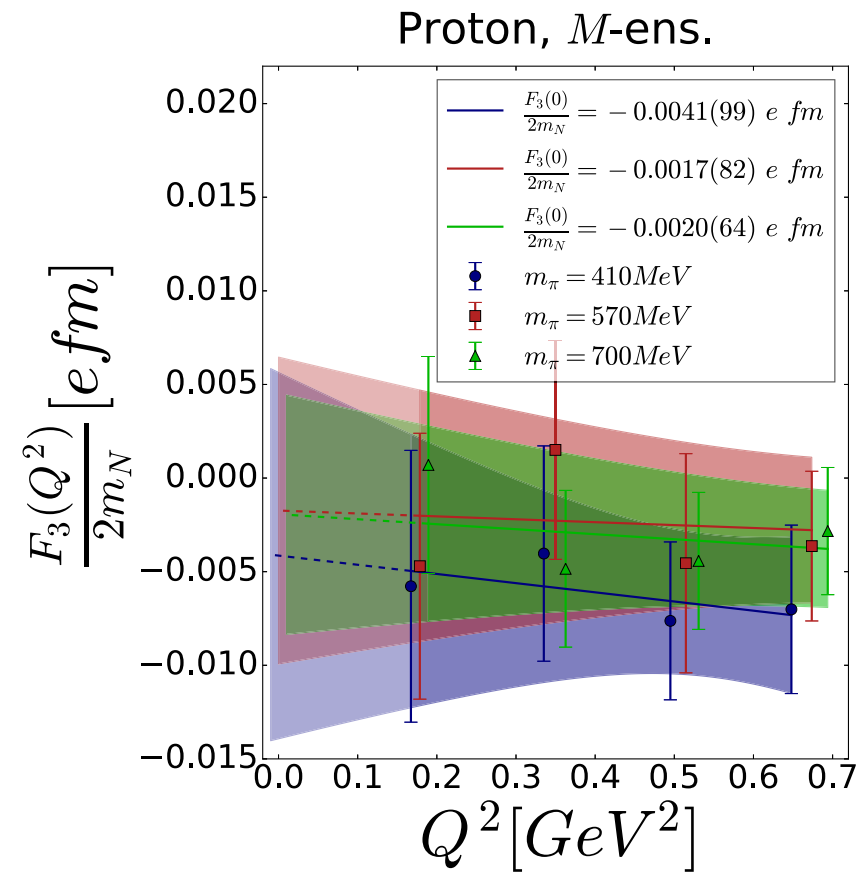

(b)

FIG. 13. M ensemble results for the neutron (left) and proton (right) CP-odd form factor $\frac{F_{3}\left(Q^{2}\right)}{2 M_{N}}$, plotted against the transfer momentum $Q^{2}$. The extrapolation to $Q^{2} \rightarrow 0$ gives the final EDMs which are displayed in Table $\mathrm{V}$.

Due to subtleties between lattice and physical quantities, the form factor decomposition in the presence of a CP-violating operator insertion is written in terms of modified form factors, $\widetilde{F}_{2}\left(Q^{2}\right)$ and $\widetilde{F}_{3}\left(Q^{2}\right)$, related to the physical form factors by [6]

$$
\begin{aligned}
& F_{3}\left(Q^{2}\right)=\cos \left(2 \alpha_{N}\right) \widetilde{F}_{3}\left(Q^{2}\right)+\sin \left(2 \alpha_{N}\right) \widetilde{F}_{2}\left(Q^{2}\right), \\
& F_{2}\left(Q^{2}\right)=-\sin \left(2 \alpha_{N}\right) \widetilde{F}_{3}\left(Q^{2}\right)+\cos \left(2 \alpha_{N}\right) \widetilde{F}_{2}\left(Q^{2}\right) .
\end{aligned}
$$

The rotated form factor $F_{3}\left(Q^{2}\right)$ corresponds to the actual electric dipole form factor as measured in experiments. From now on, we will focus on this quantity.

The ratio functions $R$ and $R^{(Q)}$ become constant, as long as the large-time approximation $T \gg t \gg \tau \gg 0$ is satisfied to ensure ground-state dominance. As the fixed-sink method is employed to compute the three-point correlation functions, a region in which this large time approximation is satisfied for $\tau$ can be found and we denote the results of the fits as

$$
\begin{aligned}
R\left(\boldsymbol{p}^{\prime}, t, \boldsymbol{q}, \tau, \Pi, \gamma_{\mu}\right) & \rightarrow R_{\mathrm{fit}}\left(\boldsymbol{p}^{\prime}, t, \boldsymbol{q}, \Pi, \gamma_{\mu}\right), \\
R^{(Q)}\left(\boldsymbol{p}^{\prime}, t, \boldsymbol{q}, \tau, \Pi, \gamma_{\mu}, t_{f}\right) & \rightarrow R_{\mathrm{fit}}^{(Q)}\left(\boldsymbol{p}^{\prime}, t, \boldsymbol{q}, \Pi, \gamma_{\mu}, t_{f}\right) .
\end{aligned}
$$

The technique for fitting these ratio functions over $\tau$ is described in Appendix B. With this construction, a system of equations can be solved for form factors $F_{i}\left(Q^{2}\right), i=1,2,3$ of the form

$$
\sum_{i=1}^{3} \mathcal{A}\left(Q^{2}\right)_{A i} F_{i}\left(Q^{2}\right)=\left\{\begin{array}{l}
R_{\mathrm{fit}}\left(\mathbf{0}, t, \boldsymbol{q}_{j}, \Pi_{k}, \gamma_{l}\right) \\
R_{\mathrm{fit}}^{(Q)}\left(\mathbf{0}, t, \boldsymbol{q}_{j}, \Pi_{k}, \gamma_{l}, t_{f}\right)
\end{array},\right.
$$

where the collective index $A$ denotes any combination of the indices $A=\{j, k, l\}$. In other words, we run over all possible combinations of projectors $\Pi$, all current momentum $\boldsymbol{q}$ within a given $Q^{2}$, and operator gamma matrix $\gamma_{\mu}$. The index $A$ of the matrix $\mathcal{A}_{A i}\left(Q^{2}\right)$ corresponds to the coefficients for each form factor $F_{i}$ for the corresponding ratio function $R$ or $R^{(Q)}$. These coefficients are found by analyzing the spectral decomposition of $R$ or $R^{(Q)}$, which needs to be done for every evaluated index $A$.

Using Eq. (21), we extrapolate to $F_{3}^{p / n}\left(Q^{2} \rightarrow 0\right) /\left(2 M_{N}\right)=$ $d_{p / n}$. We use a linear plus constant fit function, giving the extrapolated value $d_{p / n}$ at $Q^{2} \rightarrow 0$ (as well as slope in $Q^{2}$ providing $S_{p / n}$ ).

The final extraction of the neutron (left) and proton (right) $\mathrm{CP}$-odd form factor $\frac{F_{3}\left(Q^{2}\right)}{2 M_{N}}$ is shown for the $\mathrm{M}$ ensembles in Fig. 13 and for the A ensembles in Fig. 14. Figure 13 shows that all $\mathrm{M}$ ensembles are statistically consistent with each other and with zero. Figure 14 shows that there are no major discretization effects, as all the extrapolated $Q^{2} \rightarrow 0$ results are consistent.

Figures 15-17 are all displayed to understand the systematic effects resulting from varying the flow time $t_{f}$, and different methods of determining the nucleon mixing angle $\alpha_{N}$ used in the form factor decomposition $\left[\mathcal{A}_{A j}\left(Q^{2}\right)\right.$ in Eq. (35)] . In Fig. 15, for example, we show how the form factors $F_{3}$, determined at different flow-time radii $\sqrt{8 t_{f}}=0.60,0.65,0.70 \mathrm{fm}$ (green, red and blue), are statistically consistent for all three $\mathrm{M}$ ensembles (left to right). From both Fig. 16, where the improved method (see Sec. V A) of determining the nucleon mixing angle $\alpha_{N}$ (in red) is compared to the standard method for $\alpha_{N}$ (in blue), and Fig. 17, where we vary the fit range for extracting $\alpha_{N}$, it is clear that a more precise determination of $\alpha_{N}$ has a negligible impact on 


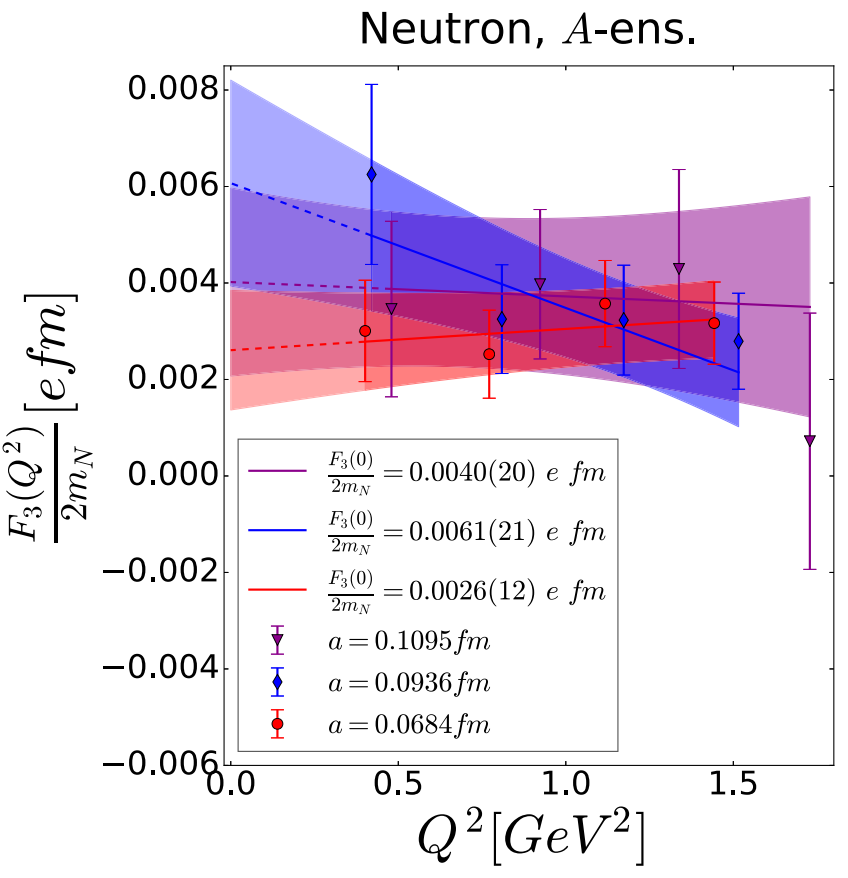

(a)

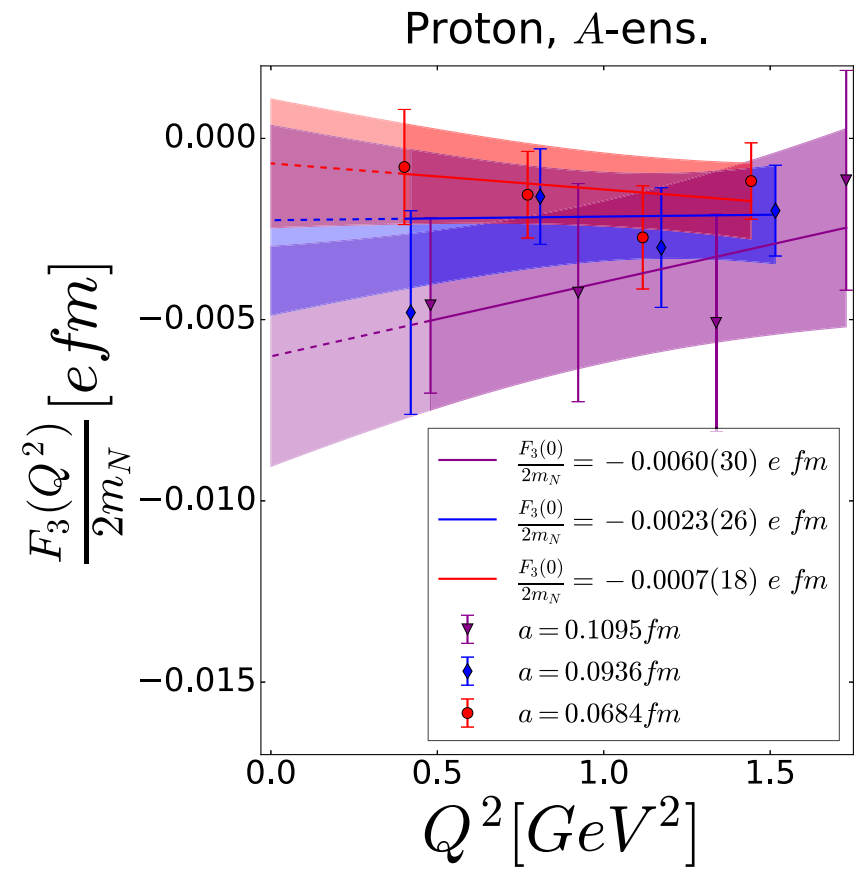

(b)

FIG. 14. A ensemble results for the neutron (left) and proton (right) CP-odd form factor $\frac{F_{3}\left(Q^{2}\right)}{2 M_{N}}$, plotted against the transfer momentum $Q^{2}$. The extrapolation to $Q^{2} \rightarrow 0$ gives the final EDMs which are displayed in Table VI.

improving the precision of the results for the $\mathrm{CP}$-odd form factor $F_{3}$. A summary of the $Q^{2} \rightarrow 0$ extrapolations for different ensembles is given in Tables $\mathrm{V}$ and VI.

\section{A. Improving the modified three-point correlation function}

In this section, we utilize a similar improvement technique used for $\alpha_{N}$, but now applied to the modified three-point correlation function $G_{3}^{(Q)}$. The improvement starts by analyzing the time dependence of the spatially integrated topological charge density

$$
\begin{aligned}
& \Delta_{3}^{(\bar{Q})}\left(\boldsymbol{p}^{\prime}, t, \boldsymbol{q}, \tau, \tau_{Q}, \Pi, \gamma_{\mu}, t_{f}\right) \\
& =a^{6} \sum_{\boldsymbol{x}, \boldsymbol{y}} e^{-i \boldsymbol{p}^{\prime} \cdot \boldsymbol{x}} e^{i \boldsymbol{q} \cdot \boldsymbol{y}} \operatorname{Tr}\{\Pi\langle\mathcal{N}(\boldsymbol{x}, t) \\
& \left.\left.\quad \times \mathcal{J}_{\mu}(\boldsymbol{y}, \tau) \bar{Q}\left(\tau_{Q}, t_{f}\right) \overline{\mathcal{N}}(\mathbf{0}, 0)\right\rangle\right\},
\end{aligned}
$$

where $\tau_{Q}$ signifies the temporal location of the topological charge $\bar{Q}$ defined in Eq. (16). The spectral decomposition for

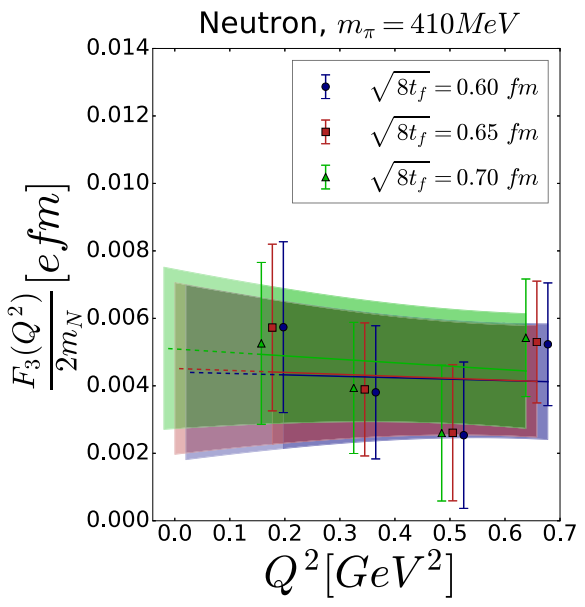

(a)

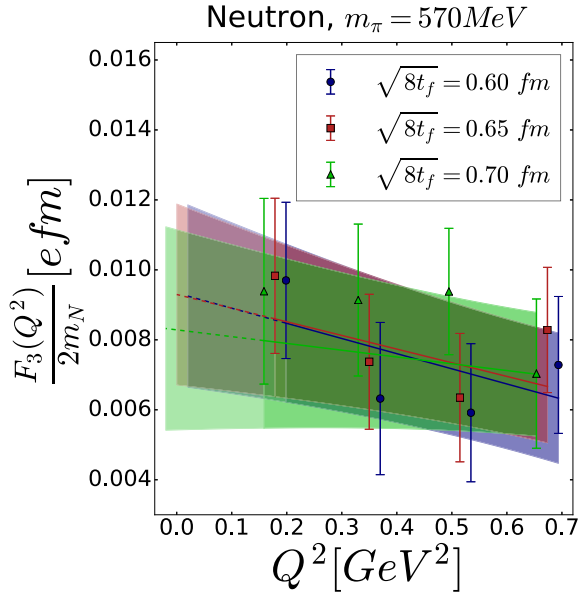

(b)

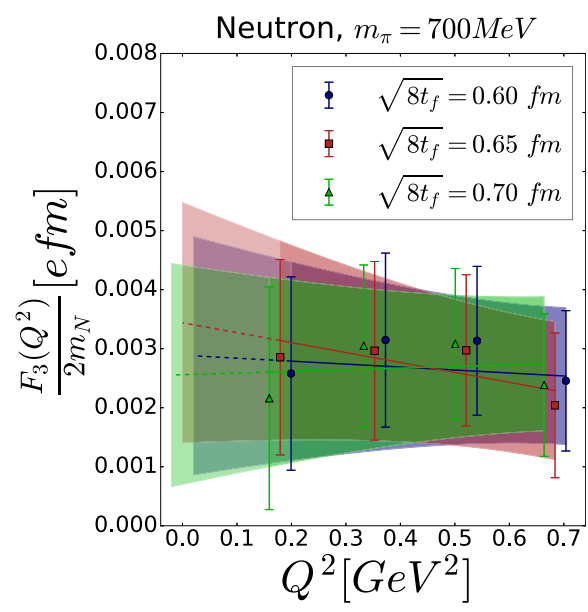

(c)

FIG. 15. Flow time radii $\sqrt{8 t_{f}}=0.60,0.65,0.70 \mathrm{fm}$ (green, red, and blue, respectively) comparison for the neutron CP-odd form factor $\frac{F_{3}\left(Q^{2}\right)}{2 M_{N}}$ using the $m_{\pi}=\{410,570,700\} \mathrm{MeV}$ (left, middle, and right, respectively) ensembles. The extrapolation to $Q^{2} \rightarrow 0$ gives the final EDM. 


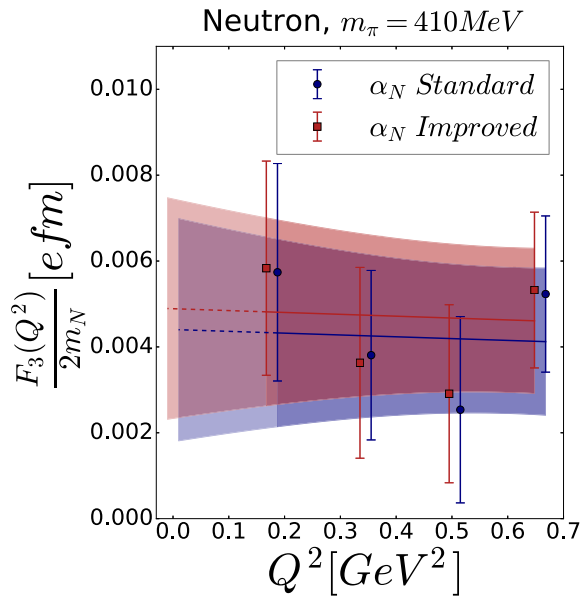

(a)

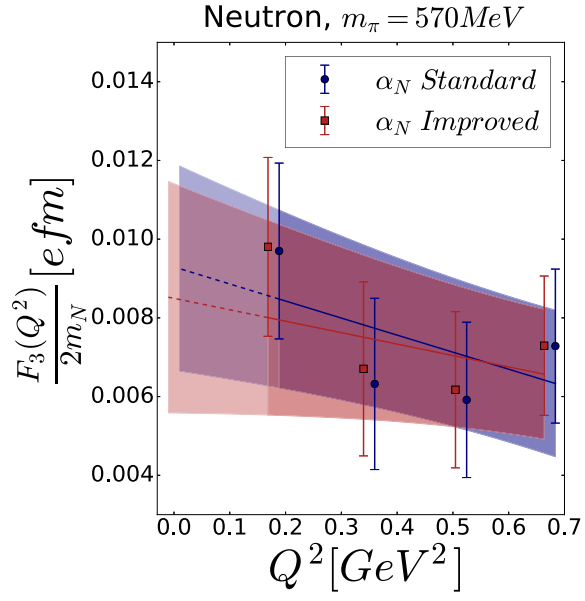

(b)

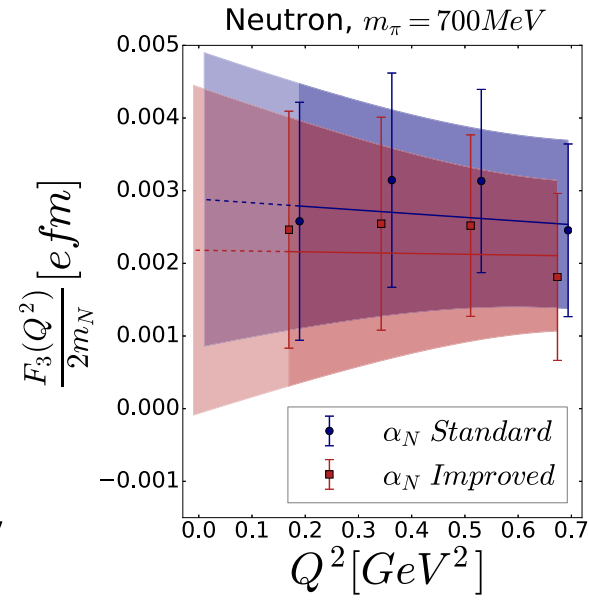

(c)

FIG. 16. Comparison of the neutron CP-odd form factor $\frac{F_{3}\left(Q^{2}\right)}{2 M_{N}}$ determined using improved (red) and unimproved (blue) results form the mixing angle $\alpha_{N}$ /Shown are the $m_{\pi}=\{410,570,700\} \mathrm{MeV}$ (left, middle, and right, respectively) ensembles. The extrapolation to $Q^{2} \rightarrow 0$ gives the final EDM.

this correlator has the form

$$
\Delta_{3}^{(\bar{Q})}= \begin{cases}\sum_{\gamma, \delta} \frac{e^{-E_{\alpha_{0}}(T-t)} e^{-E_{\beta_{0}}(t-\tau)} e^{-E_{\gamma}(\tau-\tau} Q^{\prime} e^{-E_{\delta} \tau} \tau_{Q}}{16 E_{\alpha_{0}} E_{\beta_{0}} E_{\gamma} E_{\delta}} \operatorname{Tr}\left\{\Pi\left\langle\alpha_{0}|\mathcal{N}| \beta_{0}\right\rangle\left\langle\beta_{0}\left|\mathcal{J}_{\mu}\right| \gamma\right\rangle\langle\gamma|Q| \delta\rangle\left\langle\delta|\overline{\mathcal{N}}| \alpha_{0}\right\rangle\right\}, & \tau_{Q}<\tau<t \\ \sum_{\beta, \gamma} \frac{e^{-E_{\alpha_{0}}(T-t)} e^{-E_{\beta}(t-\tau} e^{\prime} e^{-E_{\gamma}(\tau,-\tau)} e^{-E_{\delta_{0}} \tau}}{16 E_{\alpha_{0}} E_{\beta} E_{\gamma} E_{\delta_{0}}} \operatorname{Tr}\left\{\Pi\left\langle\alpha_{0}|\mathcal{N}| \beta\right\rangle\langle\beta|Q| \gamma\rangle\left\langle\gamma\left|\mathcal{J}_{\mu}\right| \delta_{0}\right\rangle\left\langle\delta_{0}|\overline{\mathcal{N}}| \alpha_{0}\right\rangle\right\}, & \tau<\tau_{Q}<t, \\ \sum_{\alpha, \beta} \frac{\left.e^{-E_{\alpha}(T-\tau}\right)^{\prime} e^{\left.-E_{\beta}(\tau)-t\right)} e^{-E_{\gamma_{0}}(t-\tau)} e^{-E_{\delta_{0} \tau} \tau}}{16 E_{\alpha} E_{\beta} E_{\gamma_{0}} E_{\delta_{0}}} \operatorname{Tr}\left\{\Pi\langle\alpha|Q| \beta\rangle\left\langle\beta|\mathcal{N}| \gamma_{0}\right\rangle\left\langle\gamma_{0}\left|\mathcal{J}_{\mu}\right| \delta_{0}\right\rangle\left\langle\delta_{0}|\overline{\mathcal{N}}| \alpha\right\rangle\right\}, & \tau<t<\tau_{Q}\end{cases}
$$

where $\alpha, \beta, \gamma$, and $\delta$ are labels for the states propagating, and the ${ }_{0}$ subscript indicates the lowest energy state propagating with the appropriate quantum numbers. We stress that $t_{f} \neq 0$ implies the absence of any contact terms. From Fig. 18, a clear signal is observed at $\tau_{Q}=0$ on all ensembles. This motivates summing $\tau_{Q}$ symmetrically around $\tau_{Q}=0$ to obtain the summed

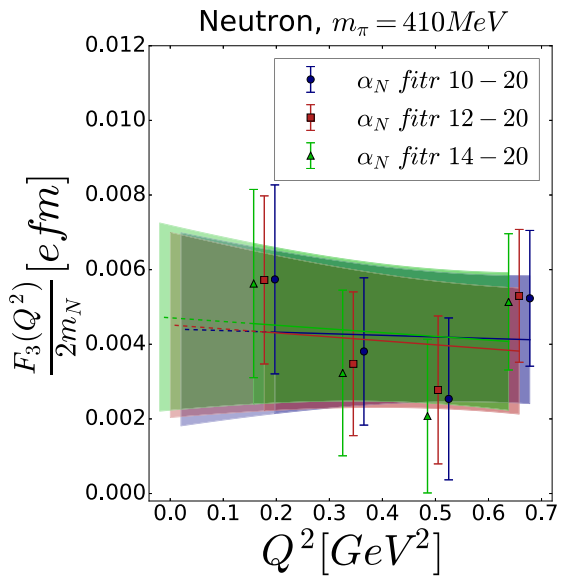

(a)

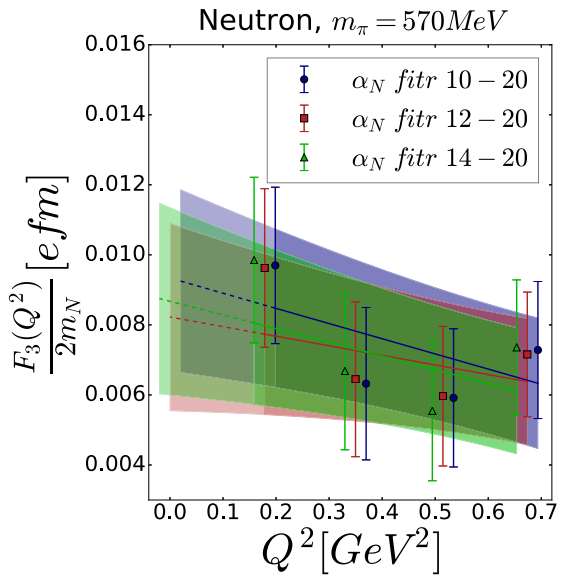

(b)

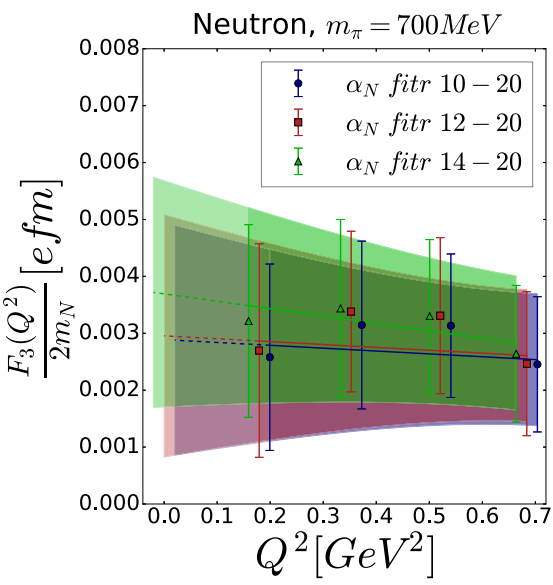

(c)

FIG. 17. Comparison of the neutron CP-odd form factor $\frac{F_{3}\left(Q^{2}\right)}{2 M_{N}}$ determined using different fit ranges for the determination of the mixing angle $\alpha_{N}$ /Shown are the $m_{\pi}=\{410,570,700\} \mathrm{MeV}$ (left, middle, and right, respectively) ensembles. The extrapolation to $Q^{2} \rightarrow 0$ gives the final EDM. 
TABLE V. $\frac{F_{3}^{p / n}\left(Q^{2} \rightarrow 0\right)}{2 M_{N}}=d_{p / n}$ fit results over M ensembles, taken from Fig. 13.

\begin{tabular}{lrrr}
\hline \hline Ensemble & $m_{\pi}=410 \mathrm{MeV}$ & $m_{\pi}=570 \mathrm{MeV}$ & $m_{\pi}=700 \mathrm{MeV}$ \\
\hline$d_{p}[e \mathrm{fm}]$ & $0.0043(99)$ & $0.0017(83)$ & $0.0016(64)$ \\
$d_{n}[e \mathrm{fm}]$ & $-0.0035(66)$ & $-0.0060(53)$ & $-0.0009(47)$ \\
\hline \hline
\end{tabular}

TABLE VI. $\frac{F_{3}^{p / n}\left(Q^{2} \rightarrow 0\right)}{2 M_{N}}=d_{p / n}$ fit results over A ensembles, taken from Fig. 14.

\begin{tabular}{lrrr}
\hline \hline Ensemble & $a=0.1095 \mathrm{fm}$ & $a=0.0936 \mathrm{fm}$ & $a=0.0684 \mathrm{fm}$ \\
\hline$d_{p}[e \mathrm{fm}]$ & $0.0060(30)$ & $0.0026(25)$ & $0.0008(18)$ \\
$d_{n}[e \mathrm{fm}]$ & $-0.0043(20)$ & $-0.0063(20)$ & $-0.0023(13)$ \\
\hline \hline
\end{tabular}

three-point correlator:

$$
\bar{G}_{3}^{(\bar{Q})}\left(\boldsymbol{p}^{\prime}, t, \boldsymbol{q}, \tau, \Pi, \gamma_{\mu}, t_{f}, t_{s}\right)=a \sum_{\frac{\tau_{Q}}{a}=0}^{t_{s} / a}\left[\Delta_{3}^{(\bar{Q})}\left(\boldsymbol{p}^{\prime}, t, \boldsymbol{q}, \tau, \tau_{Q}, \Pi, \gamma_{\mu}, t_{f}\right)+\Delta_{3}^{(\bar{Q})}\left(\boldsymbol{p}^{\prime}, t, \boldsymbol{q}, \tau, T-\tau_{Q}, \Pi, \gamma_{\mu}, t_{f}\right)\right]
$$

The resulting fit function to the sum range $t_{s}$, for the range $t_{s}>t$, is

$$
\bar{G}_{3}^{(\bar{Q})}\left(t_{s}\right)= \begin{cases}A_{0}+\sum_{\gamma_{ \pm} \neq 0_{ \pm}} A_{\gamma_{ \pm} 0_{\mp}} e^{-E_{\gamma_{ \pm}}\left(\tau-t_{s}\right)} e^{-E_{0_{\mp} t_{s}}+A_{0_{ \pm} 0_{\mp}} e^{-E_{0_{ \pm}} t_{s}} e^{-E_{0_{\mp}}\left[(T-t)-t_{s}\right]}} & 0<t_{s}<\tau \\ A_{0}+\sum_{\beta_{ \pm}, \gamma_{\mp}} A_{\beta_{ \pm} \gamma_{\mp}} e^{-E_{\beta_{ \pm}}\left(t-t_{s}\right)} e^{-E_{\gamma_{\mp}}\left(t_{s}-\tau\right)}+A_{0_{ \pm} 0_{\mp}} e^{-E_{0_{ \pm}} t_{s}} e^{-E_{0_{\mp}}\left[(T-t)-t_{s}\right]} & \tau<t_{s}<t \\ A_{0}+\sum_{\beta_{ \pm} \neq 0_{ \pm}} A_{\beta_{ \pm} 0_{\mp}} e^{-E_{0_{\mp}}\left(T-t_{s}\right)} e^{-E_{\beta_{ \pm}}\left(t_{s}-t\right)}+A_{0_{ \pm} 0_{\mp}} e^{-E_{0_{ \pm}} t_{s}} e^{-E_{0_{\mp}}\left[(T-t)-t_{s}\right]} & t<t_{s}<T / 2\end{cases}
$$

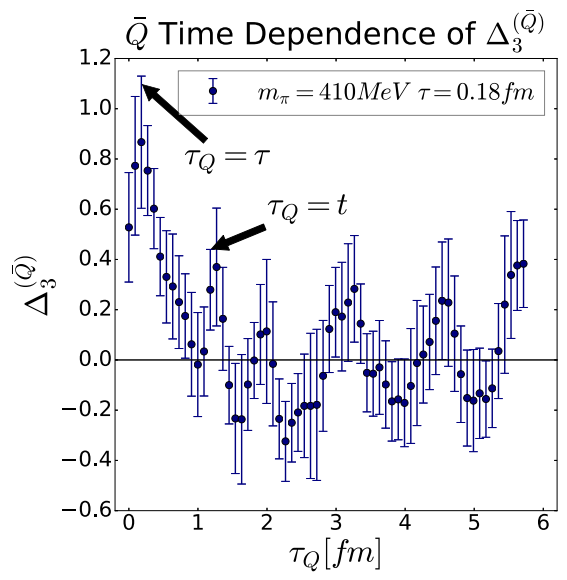

(a)

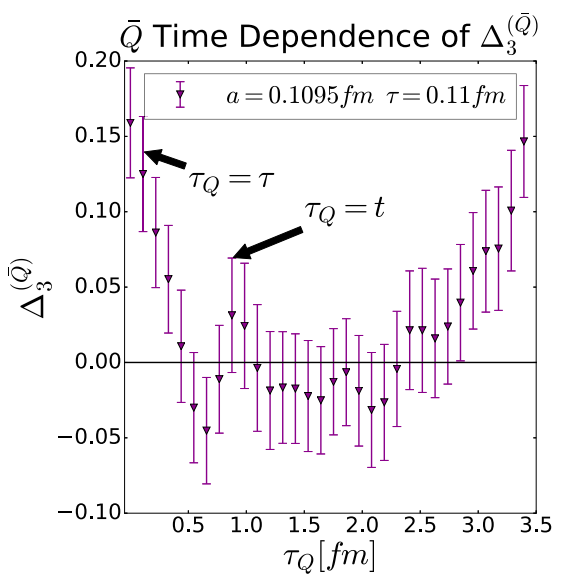

(d)

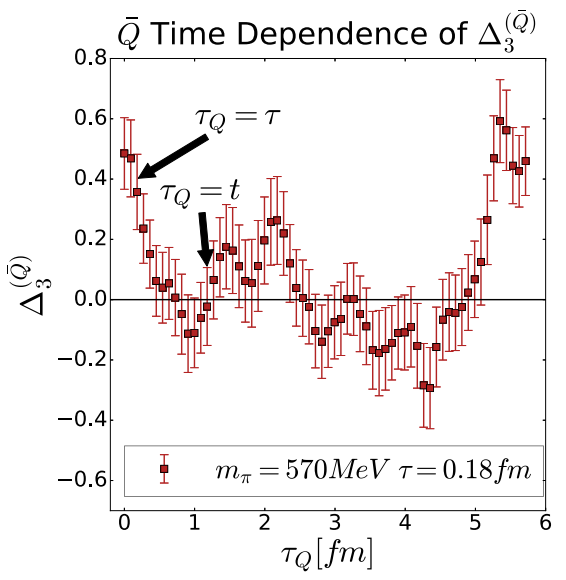

(b)

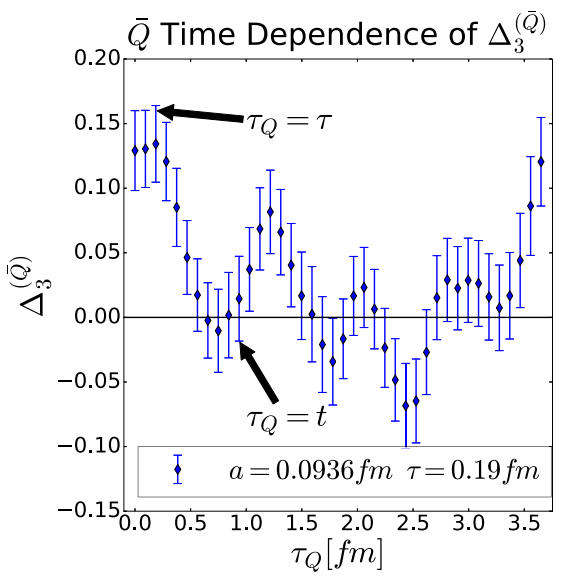

(e)

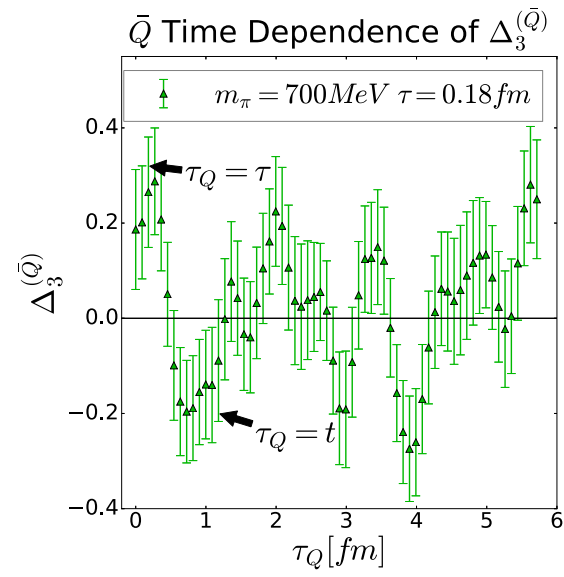

(c)

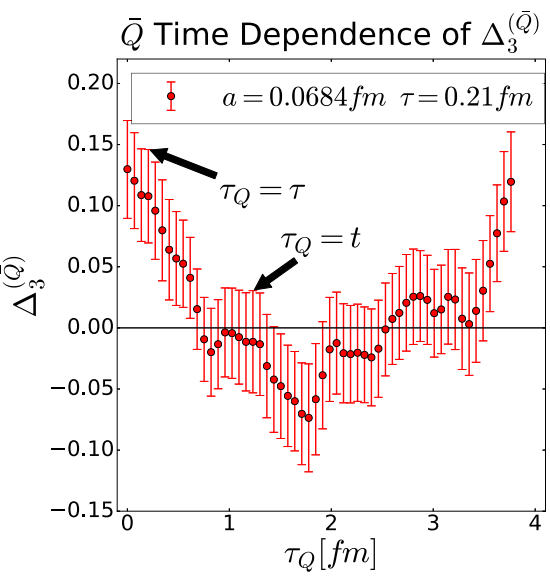

(f)

FIG. 18. Plot of the ratio $\Delta_{3}^{(\bar{Q})}$ as a function of $\tau_{Q}$, the insertion time of the topological charge $\bar{Q}\left(t_{f}, \tau_{Q}\right)$ [see Eq. (16)]. We show the result for momentum $\boldsymbol{q}=\frac{2 \pi}{L}(0,0,2), \gamma_{\mu}=\gamma_{4}, \Pi=\Pi_{+} i \gamma_{5} \gamma_{3}$ and the current insertion time $\tau$ indicated in legend. The upper left, middle, and right plots are the $m_{\pi}=\{410,570,700\} \mathrm{MeV}$ M ensembles and the lower left, middle, and right plots are the $a=\{0.1095,0.0936,0.0684\} \mathrm{fm} \mathrm{A}$ ensembles. 


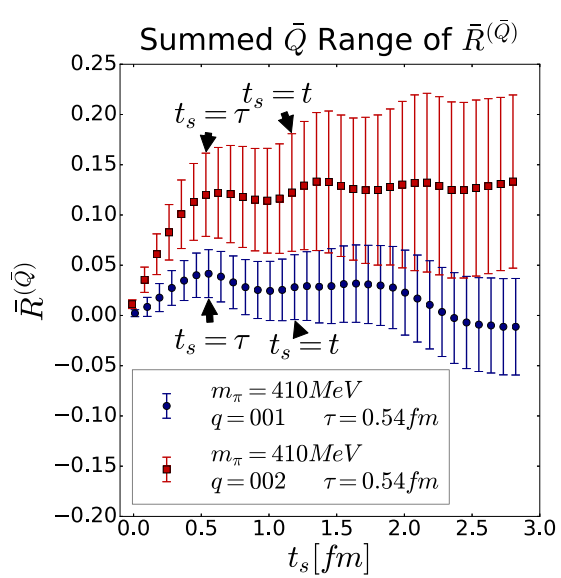

(a)

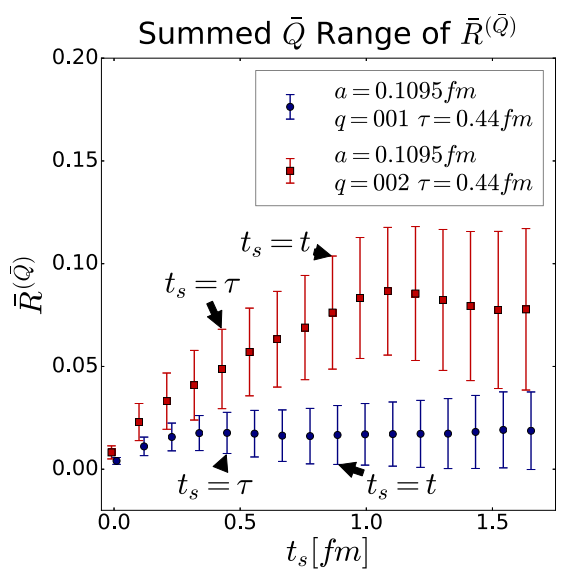

(d)

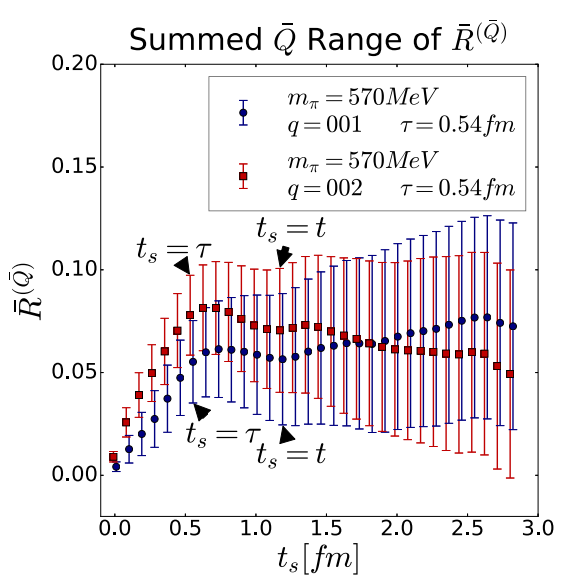

(b)

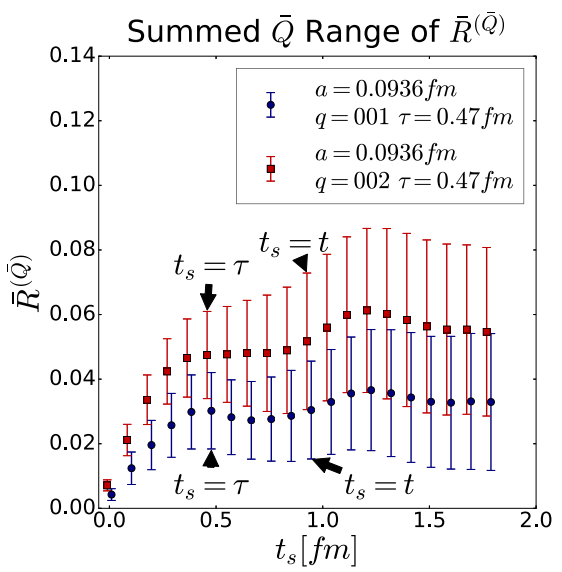

(e)

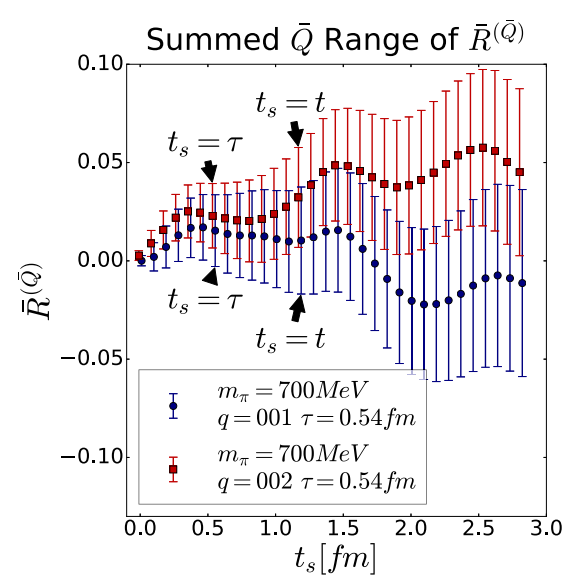

(c)

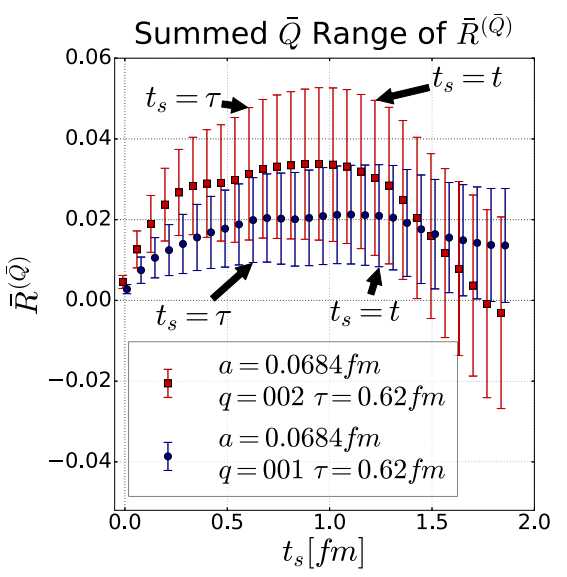

(f)

FIG. 19. Plot of the ratio function $\bar{R}^{(\bar{Q})}$ summed over $\tau_{Q}$ (see Fig. 18) from 0 to $t_{s}$ and from $T-t_{s}$ to $T$, as a function of the summation window $t_{s}$. We show the result for momentum $\boldsymbol{q}=\frac{2 \pi}{L}(0,0,2), \gamma_{\mu}=\gamma_{4}, \Pi=\Pi_{+} i \gamma_{5} \gamma_{3}$ and the current insertion time $\tau$ indicated in legend. The upper left, middle, and right plots are the $m_{\pi}=\{410,570,700\} \mathrm{MeV} \mathrm{M}$ ensembles and the lower left, middle, and right plots are the $a=\{0.1095,0.0936,0.0684\} \mathrm{fm}$ A ensembles. The standard $R^{(Q)}$ value for this quantity is obtained by taking the final $t_{s}=\frac{T}{2}$ value.

where $\gamma_{ \pm}$and $\beta_{ \pm}$represent the positive and negative $( \pm)$ parity nucleon states; $A_{0}, A_{\gamma_{ \pm}}, A_{\beta_{ \pm}, \gamma_{\mp}}$, and $A_{\beta_{ \pm}}$are combinations of nucleon matrix elements; $E_{\gamma_{ \pm}}$is the energy of the propagating state $\gamma_{ \pm}$; and $E_{0_{ \pm}}$is the lowest energy of the positive and negative parity nucleon states $0_{ \pm}$. We construct the improved ratio function $\bar{R}^{(\bar{Q})}$ in the same way as in Eq. (24), but with the replacement of $G_{3}^{(Q)} \rightarrow \bar{G}_{3}^{(\bar{Q})}$. The value of the correlation function $G_{3}^{(Q)}$, used to extract the CP-odd form factor, is obtained in the limit $t_{s} \rightarrow T$. If the summation over $\tau_{Q}$ is performed up to a value $t_{s}<T$ the neglected terms will be exponentially small as one can deduce from Eq. (39). Our numerical results seem to indicate that indeed the neglected contributions for intermediate values of $t_{s}$ are well below the statistical accuracy of our calculation.

In Fig. 19, the results for the symmetrically summed topological charge ratio function $\bar{R}^{(\bar{Q})}$ are shown as a function of the sum range $t_{s}$. In all cases, a plateau can be observed at $t_{s}=\tau$. This indicates that all the exponential terms in Eq. (39) are suppressed for $t_{s}>\tau$. Coupled with the large statistical noise inherent in the data, we fit the result with a constant value once the plateau has formed. These fit ranges are displayed in Tables VII and VIII, and are used for the form factor analysis in Sec. VIB.

TABLE VII. Fit ranges $\left[t_{s}^{\min }, \frac{T}{2}\right]$, over the symmetrically summed $\bar{Q}$ time $t_{s}$ and resulting in the improved EDM determination $\frac{F_{3}^{n}\left(Q^{2} \rightarrow 0\right)}{2 M_{N}} \equiv d_{n}$, over the $\mathrm{M}$ ensembles, taken from Fig. 21. The unimproved results from Table $\mathrm{V}$, here denoted with $\bar{d}_{n}$ to avoid confusion, are included for comparison. The values determined at $t_{s}^{\min }$ differ by the fit results at most by $10 \%$ of the error associated.

\begin{tabular}{lccc}
\hline \hline Ensemble & $m_{\pi}=410 \mathrm{MeV}$ & $m_{\pi}=570 \mathrm{MeV}$ & $m_{\pi}=700 \mathrm{MeV}$ \\
\hline Fit range & {$[6,32]$} & {$[7,32]$} & {$[4,32]$} \\
Fitr $[\mathrm{fm}]$ & {$[0.54,2.9]$} & {$[0.63,2.9]$} & {$[0.63,2.9]$} \\
$d_{n}[e \mathrm{fm}]$ & $-0.0045(26)$ & $-0.0090(27)$ & $-0.0027(20)$ \\
$\bar{d}_{n}[e \mathrm{fm}]$ & $-0.0035(66)$ & $-0.0060(53)$ & $-0.0009(47)$ \\
\hline \hline
\end{tabular}


TABLE VIII. Fit ranges $\left[t_{s}^{\min }, \frac{T}{2}\right]$, over the symmetrically summed $\bar{Q}$ time $t_{s}$ and resulting in the improved EDM determination $\frac{F_{3}^{n}\left(Q^{2} \rightarrow 0\right)}{2 M_{N}} \equiv \bar{d}_{n}$, over the A ensembles, taken from Fig. 21 . The unimproved results from Table VI, here denoted with $\bar{d}_{n}$ to avoid confusion, are included for comparison. The values determined at $t_{s}^{\min }$ differ by the fit results at most by $10 \%$ of the error associated.

\begin{tabular}{lccc}
\hline \hline Ensemble & $a=0.1095 \mathrm{fm}$ & $a=0.0936 \mathrm{fm}$ & $a=0.0684 \mathrm{fm}$ \\
\hline Fit range & {$[3,16]$} & {$[4,20]$} & {$[10,28]$} \\
Fitr $[\mathrm{fm}]$ & {$[0.36,1.9]$} & {$[0.39,2.0]$} & {$[0.69,1.9]$} \\
$d_{n}[e \mathrm{fm}]$ & $-0.0048(13)$ & $-0.00393(97)$ & $-0.0044(10)$ \\
$\bar{d}_{n}[e \mathrm{fm}]$ & $-0.0043(20)$ & $-0.0063(20)$ & $-0.0023(13)$ \\
\hline \hline
\end{tabular}

Finally, Fig. 20 displays a standard modified ratio function $R_{3}^{(Q)}$ plot over current insertion time $\tau$, where the improved ratio function (blue) is compared with the standard method (red). The improved ratio function uses the "min" time from Tables VII and VIII.
In Fig. 21, a comparison between the improved ratio functions (blue) and the standard integrated topological charge (red) used in the extraction of the neutron CP-violating form factor $\frac{F_{3}\left(Q^{2}\right)}{2 M_{N}}$ is shown. In all cases, a two- to three-fold increase in the signal-to-noise is observed and all results are statistically consistent. $^{3}$

\section{B. Continuum extrapolated results with improved ratio functions}

Armed with the improved results for the nucleon EDMs, the next step entails the extrapolation to the physical pion mass and the continuum limit. From $\chi$ PT we learn that the leading dependence of the nucleon EDMs on the pion mass is

${ }^{3}$ We have at most $1.5 \sigma$ disagreement between the two methods at $Q^{2} \rightarrow 0$ for the $a=0.0684 \mathrm{fm}$ ensemble.

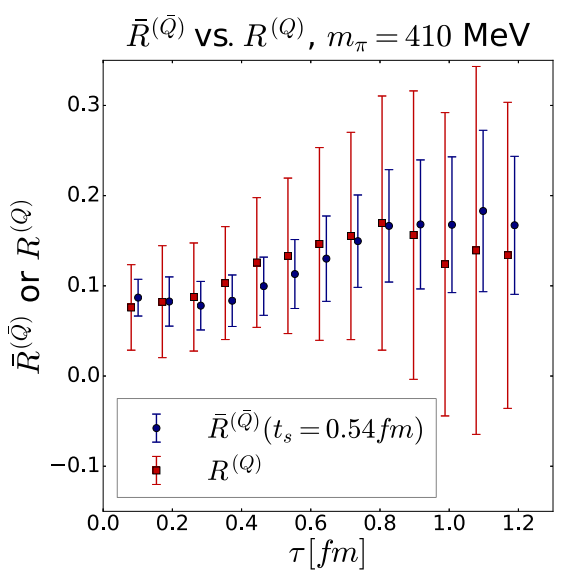

(a)

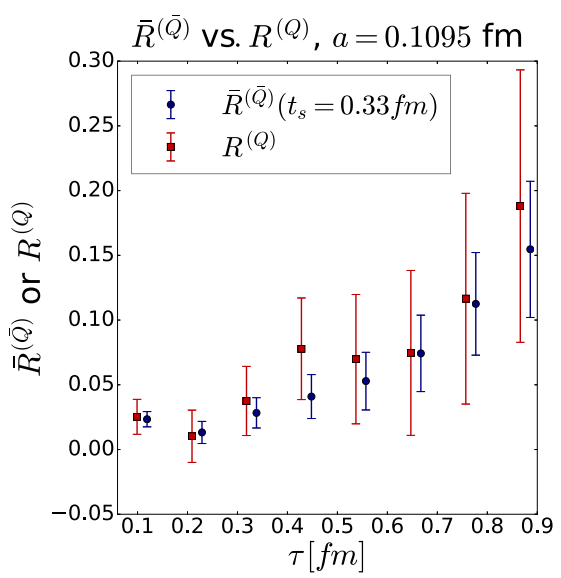

(d)

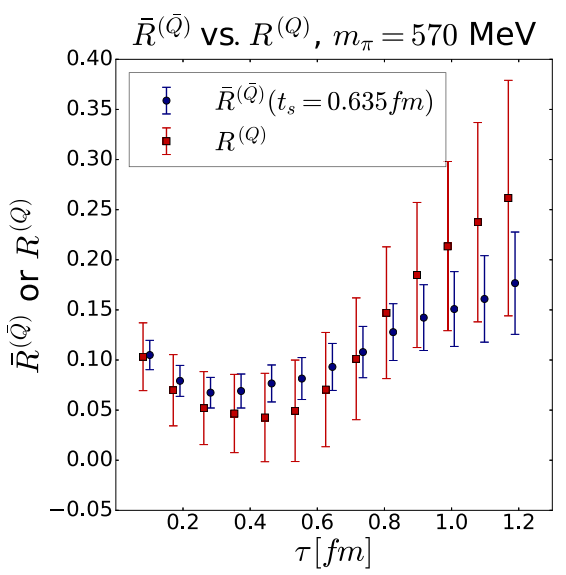

(b)

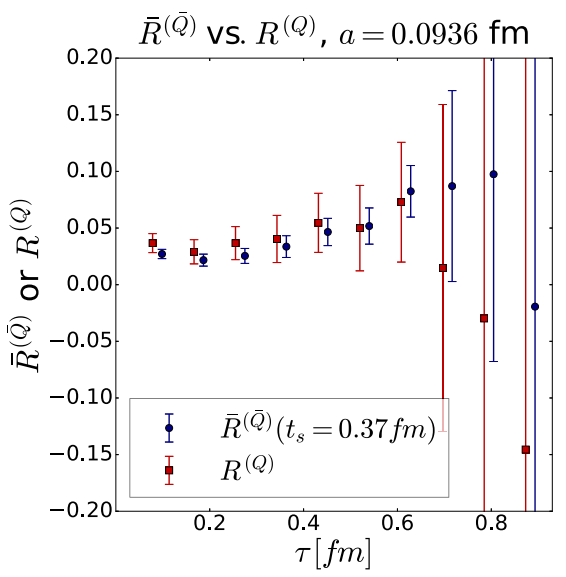

(e)

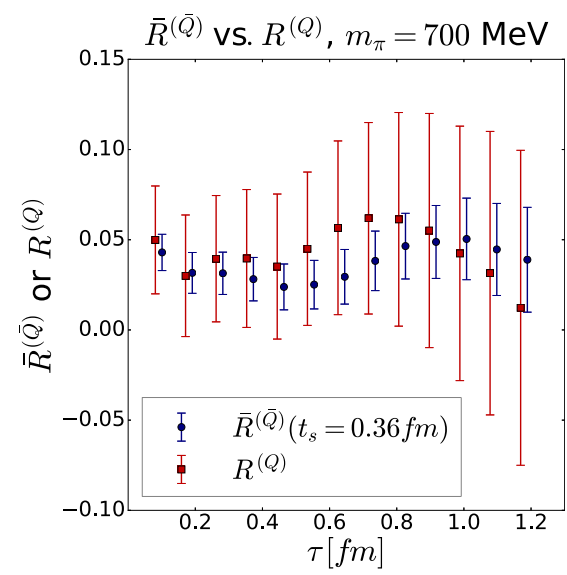

(c)

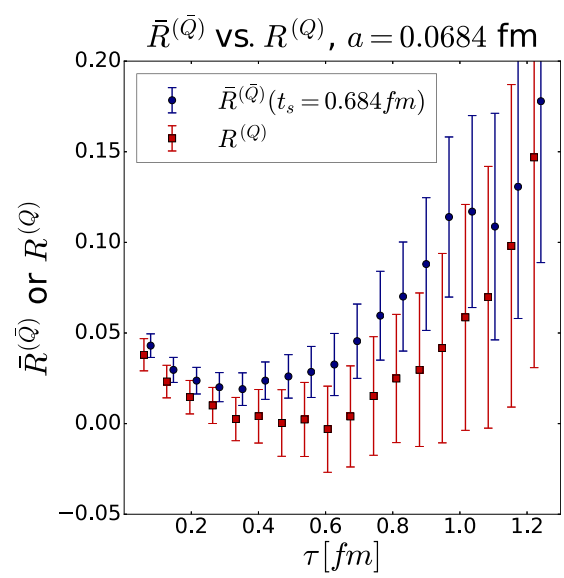

(f)

FIG. 20. Comparison of improved $\bar{R}^{(\bar{Q})}$ (blue) and unimproved $R^{(Q)}$ (red) method for computing the ratio function as a fucntion of the vector current insertion time $\tau$. We show the result for momentum $\boldsymbol{q}=\frac{2 \pi}{L}(0,0,2), \gamma_{\mu}=\gamma_{4}, \Pi=\Pi_{+} i \gamma_{5} \gamma_{3}$. The upper left, middle, and right plots are the $m_{\pi}=\{410,570,700\} \mathrm{MeV}$ M ensembles and the lower left, middle, and right plots are the $a=\{0.1095,0.0936,0.0684\} \mathrm{fm} \mathrm{A}$ ensembles. The $t_{s}$ values in the legends were selected as the $t_{s}^{\min }$ values from Tables VII and VIII. 


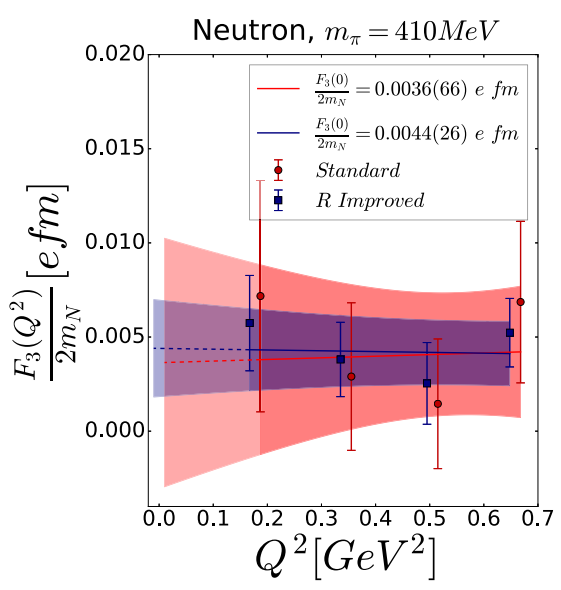

(a)

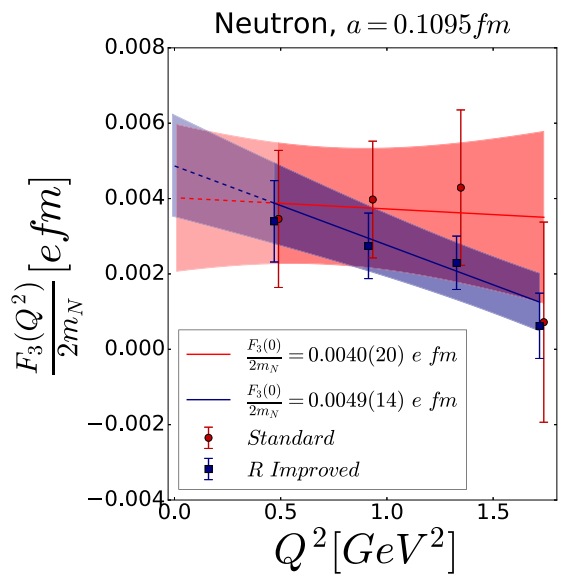

(d)

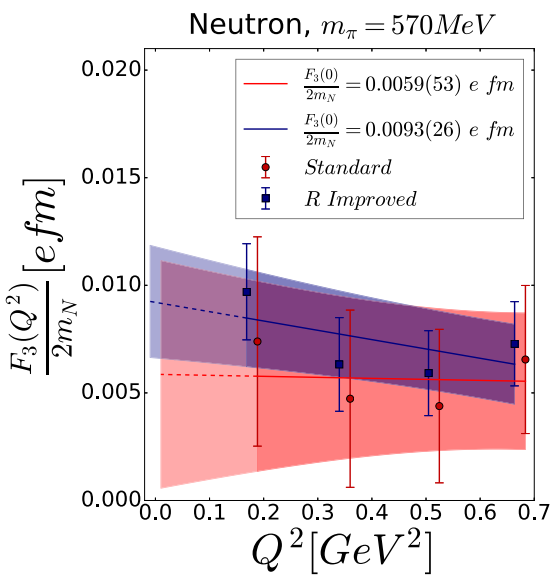

(b)

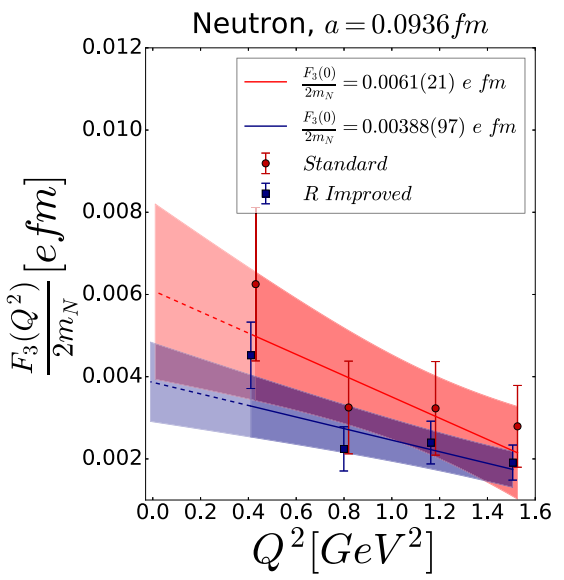

(e)

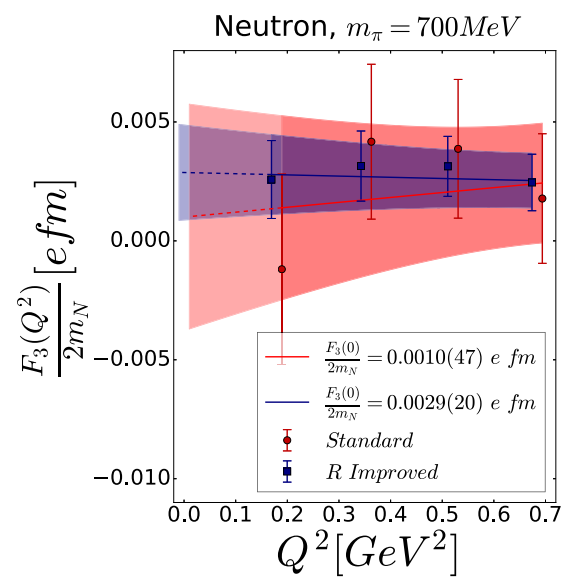

(c)

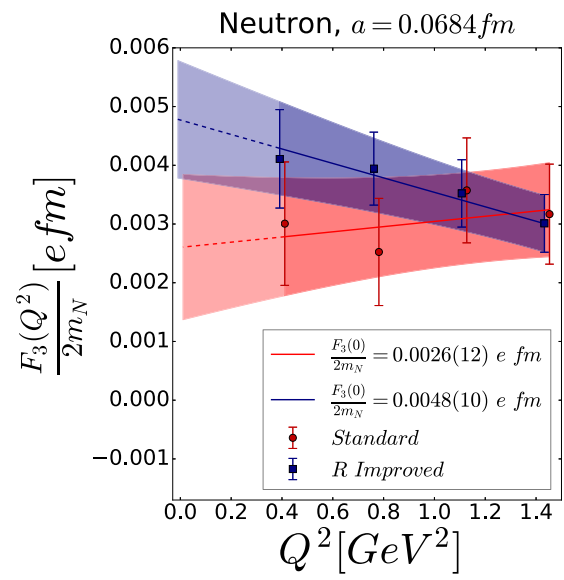

(f)

FIG. 21. The neutron CP-odd form factor $\frac{F_{3}\left(Q^{2}\right)}{2 M_{N}}$ results plotted against the transfer momentum $Q^{2}$. The $m_{\pi}$ results (upper) were computed on $m_{\pi}=\{410,570,700\} \mathrm{MeV}$ (left, middle, and right, respectively) $\mathrm{M}$ ensembles, and the lattice spacing results (lower) were computed at $a=\{0.1095,0.0936,0.0684\} \mathrm{fm}$ (left, middle, and right, respectively) A ensembles. The form factors computed with the improved ratio functions (blue) is compared with the standard ratio functions (red). The bands are linear fits to the data, which are used to extrapolate to $Q^{2} \rightarrow 0$ to determine the final EDM. Similar results are obtained for the proton.

given by [50]

$$
d_{p / n}\left(m_{\pi}\right)=C_{1} m_{\pi}^{2}+C_{2} m_{\pi}^{2} \ln \left(\frac{m_{\pi}^{2}}{m_{N, \text { phys }}^{2}}\right),
$$

where $C_{1}$ and $C_{2}$ are fit constants. To account for the finite lattice spacing, we include an additional fit parameter, $C_{3}$,

$$
d_{p / n}\left(a, m_{\pi}\right)=C_{1} m_{\pi}^{2}+C_{2} m_{\pi}^{2} \ln \left(\frac{m_{\pi}^{2}}{m_{N, \text { phys }}^{2}}\right)+C_{3} a^{2} .
$$

The additional term ensures that the EDM only vanishes in the chiral limit after taking the continuum limit. We have performed a global fit with Eq. (41) taking into account our 6 data points from ensembles $A_{1}-A_{3}$ and $M_{1}-M_{3}$. In the four plots in Figs. 22 and 23, we show the EDM results for the proton and neutron separately as function of the pion mass and lattice spacing.

Specifically, in Fig. 22 we show the extraction of the neutron (left) and proton (right) EDM plotted against their $m_{\pi}^{2}$ values (in $\mathrm{MeV}$ ). The blue band shows the extrapolation using the fit function in Eq. (41), evaluated at $d_{p / n}\left(a=0, m_{\pi}\right)$. This function evaluated at the physical pion mass is what we are interested in. In red we show the same extrapolation, where the fit is evaluated instead at $d_{p / n}\left(a=0.09 \mathrm{fm}, m_{\pi}\right)$, to study the role of discretization errors. In particular, we observe an uncertainty of the EDMs at the physical pion mass that is roughly twice larger at $a=0.09 \mathrm{fm}$. It is perhaps surprising that the uncertainty at the physical point reduces in the continuum limit. But the reason is clear. By fitting the nucleon EDMs to the fit function in Eq. (41), the uncertainty on the fit parameters $C_{1}$ and $C_{2}$ is increased by the presence of the $C_{3}$ term. Now that the $a^{2}$ dependence is taken into account, we can perform an interpolation between the EDM in the chiral limit and the pion masses of our ensembles. In the continuum limit, $a=0$, the resulting nucleon EDM at the physical pion mass has now less uncertainty because $d_{n, p}\left(a=0, m_{\pi}=0\right) \quad$ while $d_{n, p}\left(a>0, m_{\pi}=0\right) \neq 0 \quad$ and unconstrained. 


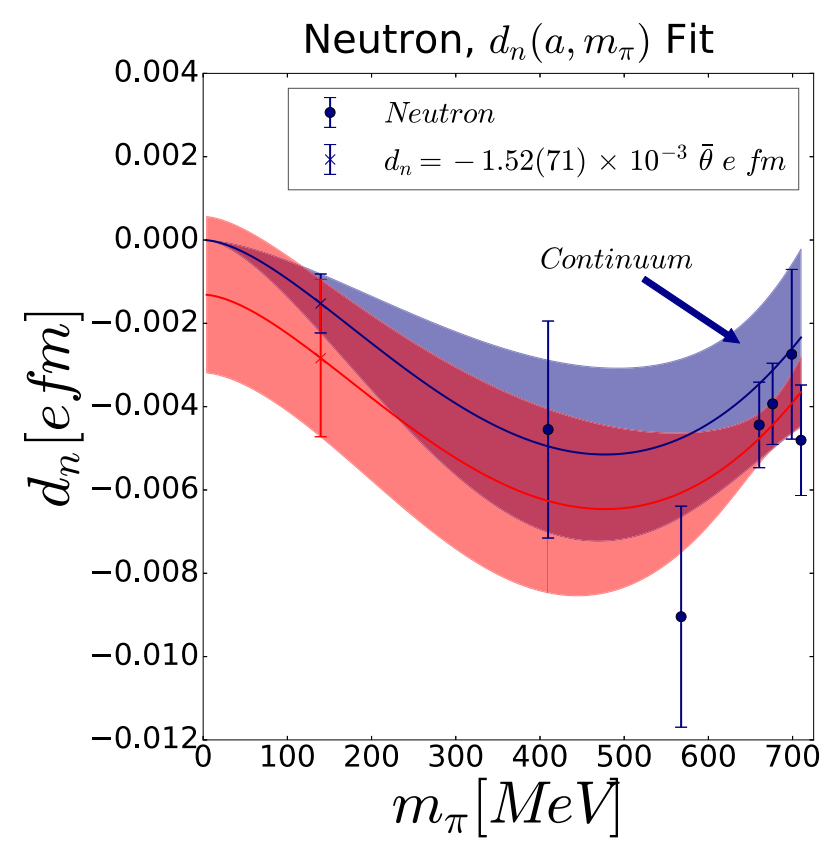

(a)

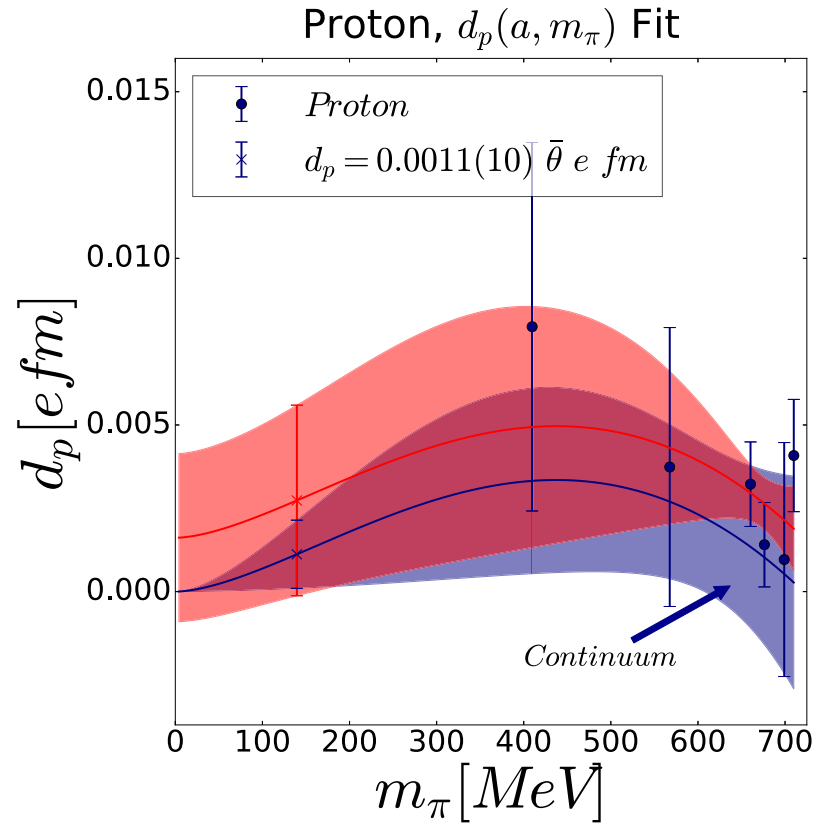

(b)

FIG. 22. Determination of the EDM $d_{p / n}$ for the neutron (left) and proton (right) for all six of our ensembles, plotted against their respective $m_{\pi}$ values. The bands are the fits to all the ensembles using Eq. (41), evaluated in the continuum $a=0$ (blue) and at $a=0.0907 \mathrm{fm}$ (red) which coincides with the lattice spacing of the $\mathrm{M}$ ensembles.

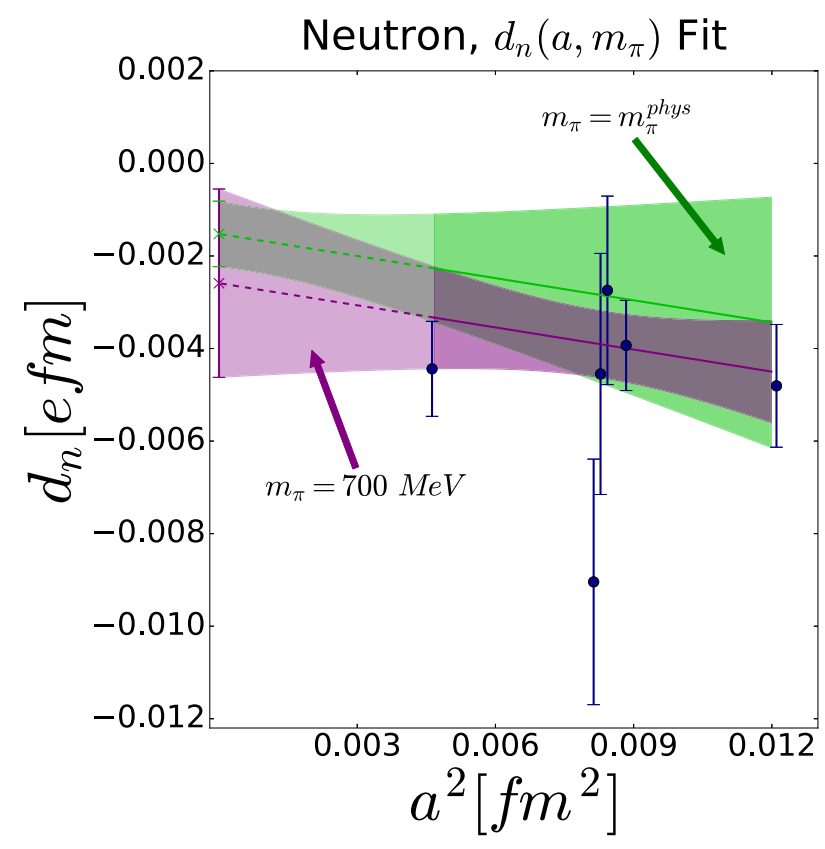

(a)

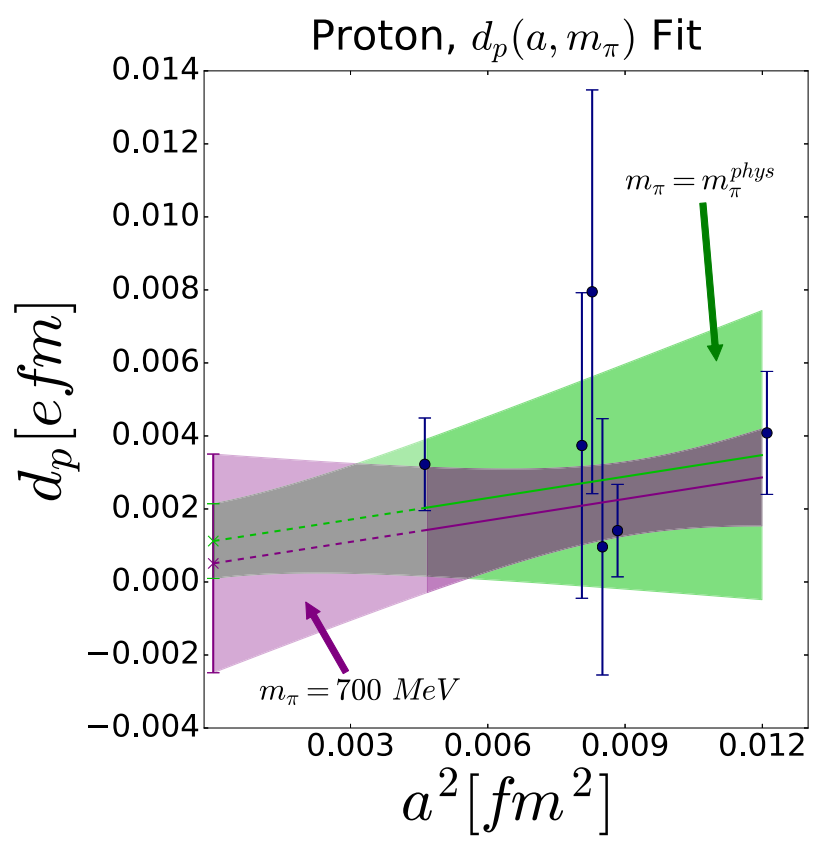

(b)

FIG. 23. Determination of the EDM $d_{p / n}$ for the neutron (left) and proton (right) for all 6 of our ensembles, plotted against their respective lattice spacing values. The bands are the fits to all the ensembles using Eq. (41), evaluated at the physical point, $m_{\pi}=m_{\pi}^{\text {phys }},($ green) and in the chiral limit $m_{\pi}=700 \mathrm{MeV}$ (purple). 
TABLE IX. Neutron and proton EDM fit parameters $C_{1}, C_{2}, C_{3}$ extracted from the combine fits to all six ensembles using Eq. (41), as well as the resulting $\chi_{\mathrm{PDF}}^{2}$. We also estimate $\bar{g}_{0}$ using $C_{2}$ and Eq. (3).

\begin{tabular}{lrrrrr}
\hline \hline & $C_{1}\left[\bar{\theta} e \mathrm{fm}^{3}\right]$ & \multicolumn{1}{c}{$C_{2}\left[\bar{\theta} e \mathrm{fm}^{3}\right]$} & $C_{3}\left[\frac{\bar{\theta} e f \mathrm{fm}}{\mathrm{fm}^{2}}\right]$ & $\chi_{\mathrm{PDF}}^{2}$ & $\bar{g}_{0}^{\bar{\theta}}[\bar{\theta}]$ \\
\hline Proton & $-3.6(5.3) \times 10^{-4}$ & $-6.8(6.6) \times 10^{-4}$ & $0.20(31)$ & $2.0(1.4)$ & $-9.9(9.6) \times 10^{-3}$ \\
Neutron & $3.1(3.2) \times 10^{-4}$ & $8.8(4.4) \times 10^{-4}$ & $-0.16(23)$ & $1.8(1.5)$ & $-12.8(6.4) \times 10^{-3}$ \\
\hline \hline
\end{tabular}

The final continuum extrapolation values for the neutron and proton EDM are

$$
\begin{aligned}
& d_{n}\left(a=0, m_{\pi}=m_{\pi}^{\text {phys }}\right)=-0.00152(71) \bar{\theta} e \mathrm{fm}, \\
& d_{p}\left(a=0, m_{\pi}=m_{\pi}^{\text {phys }}\right)=0.0011(10) \bar{\theta} e \mathrm{fm},
\end{aligned}
$$

and we include the determination for the fit parameters $C_{1}$, $C_{2}, C_{3}$ of Eq. (41), as well as the $\chi$-squared per degree of freedom parameter, $\chi_{\mathrm{PDF}}^{2}$, in Table IX. The error on $\chi_{\mathrm{PDF}}^{2}$ is determined from the bootstrap samples distribution. Since the correlators for the proton and the neutron EDM are different, it is possible to obtain different relative uncertainties in the two cases. It is not clear to us though, why we observe a relative larger uncertainty for the proton than for the neutron.

In Fig. 23 we show the dependence of our EDM results on the lattice spacing $a$ for the neutron (left) and proton (right) EDM. Overlaid on top, we have the evaluation of the fit function Eq. (41) at two different values of $m_{\pi}: m_{\pi}=700 \mathrm{MeV}$ (purple band) and $m_{\pi}=m_{\pi}^{\text {phys }}$ (green band). The ensembles analyzed in this work do not allow us to study mass-dependent discretization effects, but we can still observe the impact of the chiral interpolation on the continuum limit. The continuum extrapolation has less uncertainty, thanks to the constraint that the EDM vanishes in the chiral limit. Adding more ensembles to study mass-dependent cutoff effects is certainly desirable, but it does not change the main conclusion of this analysis.

We can extract a value of the CP-odd pion-nucleon LEC, $\bar{g}_{0}$, which plays an important role in the EDMs of nuclei and diamagnetic atoms, by identifying our result for fit parameter $C_{2}$ with the coefficient of the log term in Eq. (3) for the neutron EDM. This gives the relation

$$
\bar{g}_{0}=-\frac{8 \pi^{2} f_{\pi}}{g_{A}} \frac{C_{2} m_{\pi}^{2}}{e},
$$

leading to the extraction

$$
\bar{g}_{0}=-12.8(6.4) \times 10^{-3} \bar{\theta},
$$

at the physical pion mass. As discussed in Sec. II [see Eq. (5)] the LEC $\bar{g}_{0}$ can also be extracted from a completely independent calculation relating the proton-neutron mass splitting, induced purely by QCD effects, to the $\theta$ term $[20,33,34]$. The value of $\bar{g}_{0}$ we obtain from our EDM calculation, Eq. (44), is perfectly consistent within statistical uncertainties with the result obtained from the proton-neutron mass difference we rewrite here for convenience,

$$
\left[\bar{g}_{0}\right]_{p-n}=-14.7(2.3) \times 10^{-3} \bar{\theta} .
$$

This agreement gives us confidence that the lattice data are consistent with the pion-mass dependence predicted by chiral perturbation theory.
To assess potential sources of systematic effects that become relevant once the precision of these types of calculations reach the percent level, we perform an additional polynomial fit of the pion-mass dependence of the EDM. In the left plot of Fig. 24 we show the different contributions to the EDM pion mass dependence using $\chi \mathrm{PT}$ [see Eq. (40)] and in the right plot of Fig. 24 we show a polynomial fit to the form

$$
d_{n}\left(m_{\pi}\right)=C_{1} m_{\pi}^{2}+C_{2} m_{\pi}^{4}
$$

The lattice data are equally well described by both expressions as the resulting $\chi_{\text {pdf }}^{2}$ for the polynomial fits are

$$
\begin{array}{ll}
\chi_{\mathrm{pdf}}^{2}=1.7(1.4), & \text { neutron, } \\
\chi_{\mathrm{pdf}}^{2}=2.0(1.4), & \text { proton, }
\end{array}
$$

to be compared with the results in Table IX. We note that while in the chiral fit there is a dominance of the chiral log with a smaller contribution from the counterterm $C_{1}$ (as expected by phenomenology going back to Crewther et al. in 1979 [20]), in the polynomial fit we have the classical behavior of a potentially nonconverging expansion where the leading and next-to-leading contributions have similar sizes in absolute value and opposite signs. Nevertheless the two expressions give statistically consistent values for the EDM at the physical point

$$
\begin{aligned}
& {\left[d_{n}\right]_{\chi P T}=-1.52(71) \times 10^{-3} \bar{\theta} e \mathrm{fm},} \\
& {\left[d_{n}\right]_{\mathrm{poly}}=-0.64(25) \times 10^{-3} \bar{\theta} e \mathrm{fm},}
\end{aligned}
$$

and the EDM from the polynomial fit has even a smaller statistical uncertainty. However, the polynomial fit is not motivated by physics, whereas the chiral fit has a first-principle derivation. Combined with the striking agreement with our extraction of $\bar{g}_{0}$ with a completely independent determination (see above), it seems best to describe the data using chiral perturbation theory with the more conservative uncertainty in Eq. (42).

\section{Schiff moment of the proton and neutron}

Apart from the EDMs of the neutron and the proton, the nucleon electric dipole form factor (EDFF) contains additional information. The EDFF can be decomposed as

$$
\frac{F_{3}^{p / n}\left(Q^{2}\right)}{2 M_{N}}=d_{p / n}-S_{p / n} Q^{2}+H_{p / n}\left(Q^{2}\right),
$$

where $d_{p / n}$ denotes the proton or neutron EDM, $S_{p / n}$ denotes the proton or neutron Schiff moments defined by $S_{p / n}=\left.\left(2 M_{N}\right)^{-1}\left(d F_{3}^{p / n} / d Q^{2}\right)\right|_{Q^{2}=0}$, and $H_{p / n}$ are functions 

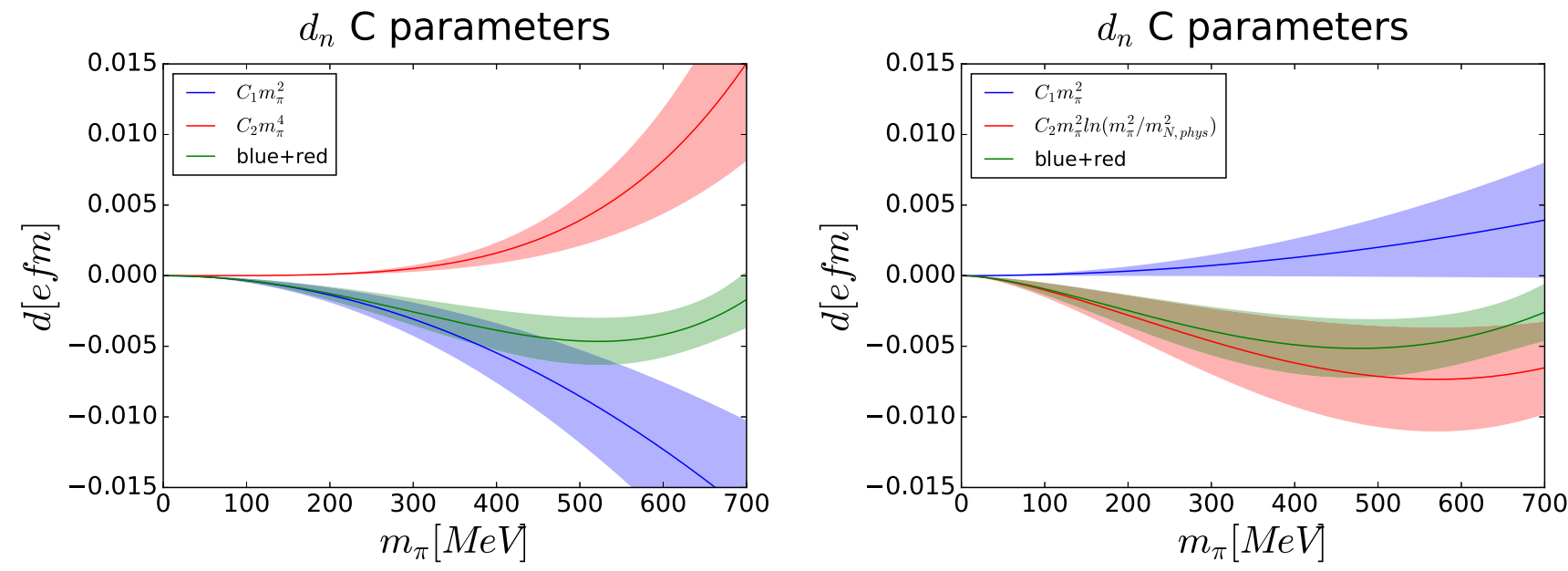

FIG. 24. Left plot: Graphical representation of the chiral fit results. The green band represents the result of the chiral fit in Eq. (40). The blue and red bands represents the separate contributions to the chiral fit, respectively, the contribution proportional to $C_{1}$ (blue) and the contribution proportional to $C_{2}$ (red band). Right plot: Same plot description as the left plot. The contributions now though refer to the polynomial fit in Eq. (46).

that capture the remaining $Q^{2}$ dependence. Chiral perturbation theory allows for a calculation of the Schiff Moments and the $H_{p / n}$ functions from the analogous isovector and isoscalar quantities [50]. At leading order in the chiral expansion the nucleon EDMs are given in Eq. (3). The leading-order Schiff moments are isovector and given by

$$
S_{p}=-S_{n}=-\frac{e g_{A} \bar{g}_{0}}{48 \pi^{2} F_{\pi} m_{\pi}^{2}}=1.7(3) \times 10^{-4} \bar{\theta} e \mathrm{fm}^{3},
$$

where we have used Eq. (5). NLO corrections have been calculated in Ref. [23] and reduce the leading-order result by roughly $50 \%$ and provide a tiny contribution to $S_{n}+S_{p}=$ $\mathcal{O}\left(10^{-5} \bar{\theta} e \mathrm{fm}^{3}\right) . \chi \mathrm{PT}$ thus predicts that the neutron and proton Schiff moments are equal in magnitude but with opposite sign. The leading-order $H_{p / n}$ are also isovector and given by

$$
H_{p}=-H_{n}=-\frac{e g_{A} \bar{g}_{0}}{30 \pi^{2} F_{\pi}}\left[h_{1}^{(0)}\left(\frac{Q^{2}}{4 m_{\pi}^{2}}\right)\right],
$$

where

$$
h_{1}^{(0)}(x)=-\frac{15}{4}\left[\sqrt{1+\frac{1}{x}} \ln \left(\frac{\sqrt{1+1 / x}+1}{\sqrt{1+1 / x}-1}\right)-2\left(1+\frac{x}{3}\right)\right] \text {. }
$$

In the limit $Q^{2} \ll m_{\pi}^{2}$ the nucleon EDFFs become

$$
\begin{aligned}
& \frac{F_{3}^{p}\left(Q \ll m_{\pi}\right)}{2 M_{N}}=d_{p}+\frac{e g_{A} \bar{g}_{0}}{48 \pi^{2} F_{\pi}}\left(\frac{Q^{2}}{m_{\pi}^{2}}+\cdots\right), \\
& \frac{F_{3}^{n}\left(Q \ll m_{\pi}\right)}{2 M_{N}}=d_{n}-\frac{e g_{A} \bar{g}_{0}}{48 \pi^{2} F_{\pi}}\left(\frac{Q^{2}}{m_{\pi}^{2}}+\cdots\right),
\end{aligned}
$$

such that the Schiff moments provide the dominant $Q^{2}$ dependence of the EDFFs. The nucleon EDMs and the LEC $\bar{g}_{0}$ are induced by the $\bar{\theta}$ term and scale as $d_{p / n} \sim \bar{g}_{0} \sim \bar{m}_{*} \bar{\theta} \sim m_{\pi}^{2} \bar{\theta}$. As such, the Schiff moments scale as $S_{p / n} \sim \bar{g}_{0} / m_{\pi}^{2}$ which is pion mass independent. This statement is potentially confusing as we infer from Eq. (2) that the $\bar{\theta}$ term decouples in the chiral limit and the whole nucleon EDFF should vanish.
Equation (55), however, requires $Q^{2} \ll m_{\pi}^{2}$. In the opposite limit, we obtain

$$
\begin{aligned}
& \frac{F_{3}^{p}\left(Q \gg m_{\pi}\right)}{2 M_{N}}=d_{p}-\frac{e g_{A} \bar{g}_{0}}{8 \pi^{2} F_{\pi}}\left(2+\ln \frac{m_{\pi}^{2}}{Q^{2}}\right), \\
& \frac{F_{3}^{n}\left(Q \gg m_{\pi}\right)}{2 M_{N}}=d_{n}+\frac{e g_{A} \bar{g}_{0}}{8 \pi^{2} F_{\pi}}\left(2+\ln \frac{m_{\pi}^{2}}{Q^{2}}\right),
\end{aligned}
$$

and the EDFFs vanish in the chiral limit as expected.

The goal is to extract $S_{p / n}$ from our lattice data as this allows for a direct comparison to the $\chi \mathrm{PT}$ prediction in Eq. (51) and the extraction in the previous section based on the pion mass dependence of the nucleon EDMs. To extract $S_{p / n}$, we first extrapolate our results to small $Q^{2}$ by fitting the EDFF to the function

$$
\begin{aligned}
\frac{F_{3}^{p / n}\left(Q^{2}, m_{\pi}^{2}, a^{2}\right)}{2 M_{N}}= & d_{p / n}\left(m_{\pi}^{2}, a^{2}\right)-S_{p / n}\left(m_{\pi}^{2}, a^{2}\right) \\
& \times\left[Q^{2}-\frac{8 m_{\pi}^{2}}{5} h_{1}^{(0)}\left(\frac{Q^{2}}{4 m_{\pi}^{2}}\right)\right] .
\end{aligned}
$$

The effects of the $h_{1}^{(0)}$ function turns out to have minimal impact on the extraction of $d_{p / n}\left(m_{\pi}^{2}, a^{2}\right)$ and $S_{p / n}\left(m_{\pi}^{2}, a^{2}\right)$, and we obtain similar results if we use the fit function

$$
\frac{F_{3}^{p / n}\left(Q^{2}, m_{\pi}^{2}, a^{2}\right)}{2 M_{N}}=d_{p / n}\left(m_{\pi}^{2}, a^{2}\right)-S_{p / n}\left(m_{\pi}^{2}, a^{2}\right) Q^{2} .
$$

This shows that our results are not precise enough to isolate the more subtle $Q^{2}$ behavior.

Once we have obtained $S_{p / n}\left(m_{\pi}^{2}, a^{2}\right)$ we can extrapolate to the continuum limit and the physical pion mass. LO $\chi \mathrm{PT}$ predicts no dependence on the pion mass, and, having an $\mathcal{O}(a)$ improved lattice action, we add a quadratic dependence on the lattice spacing $a$,

$$
S_{p / n}\left(m_{\pi}^{2}, a^{2}\right)=C_{4}+C_{5} a^{2},
$$

with $C_{4}$ and $C_{5}$ fit constants. The results for the Schiff moments along with the continuum extrapolation are shown in 


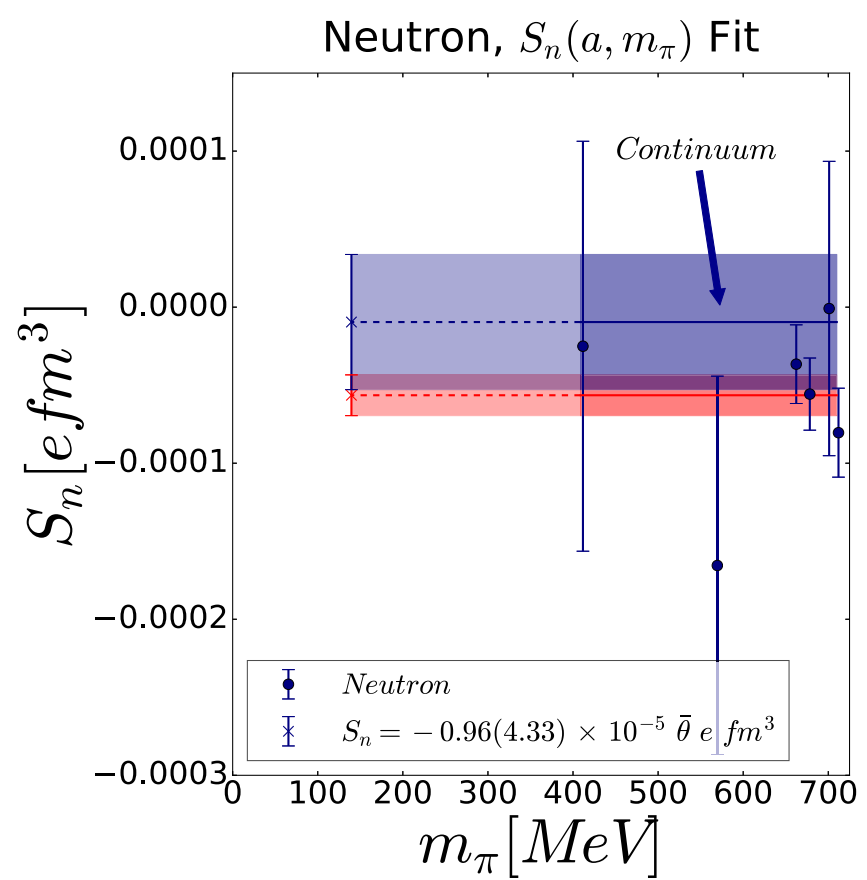

(a)

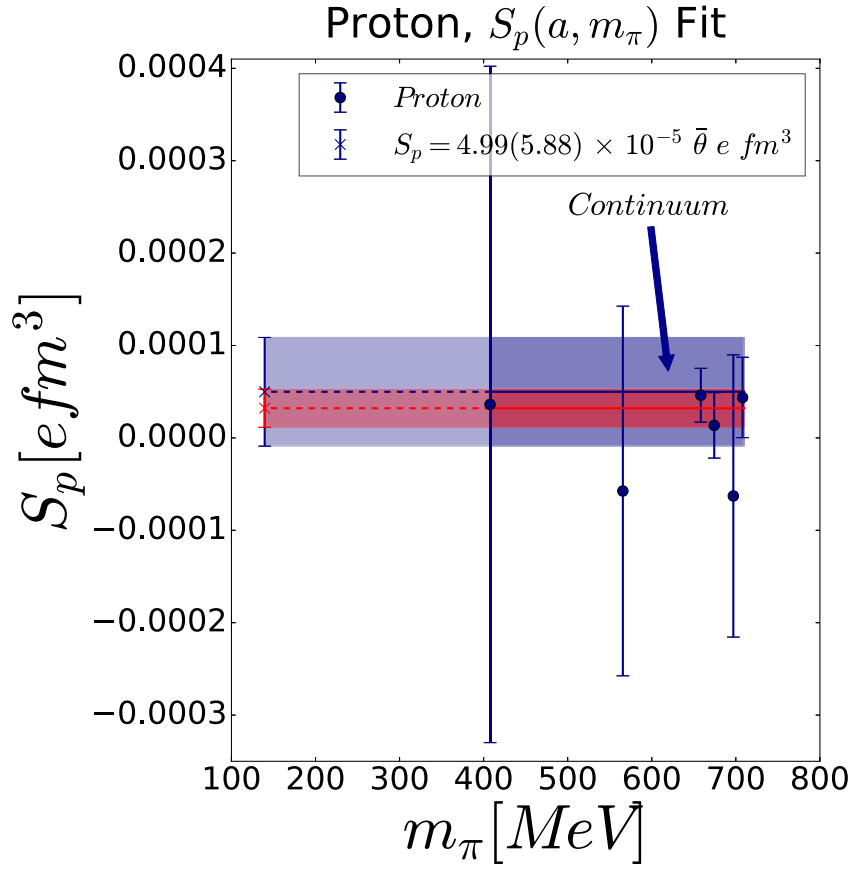

(b)

FIG. 25. Determination of the Schiff moment $S_{p / n}$ for the neutron (left) and proton (right) for all six of our ensembles, plotted against their respective $m_{\pi}$ values. The bands are the fits to all the ensembles using Eq. (60), evaluated in the continuum limit $a=0$ (blue) and the lattice spacing corresponding to the $\mathrm{M}$ ensembles (red).

Figs. 25 and 26. In Fig. 26 we show the fit results with the $a^{2}$ dependence. We observe minimal discretization effects over the range $a=\{0 \rightarrow 0.12\} \mathrm{fm}$. In Fig. 25 we show the fit results as a function of the pion mass $m_{\pi}$. At $a=0$ we

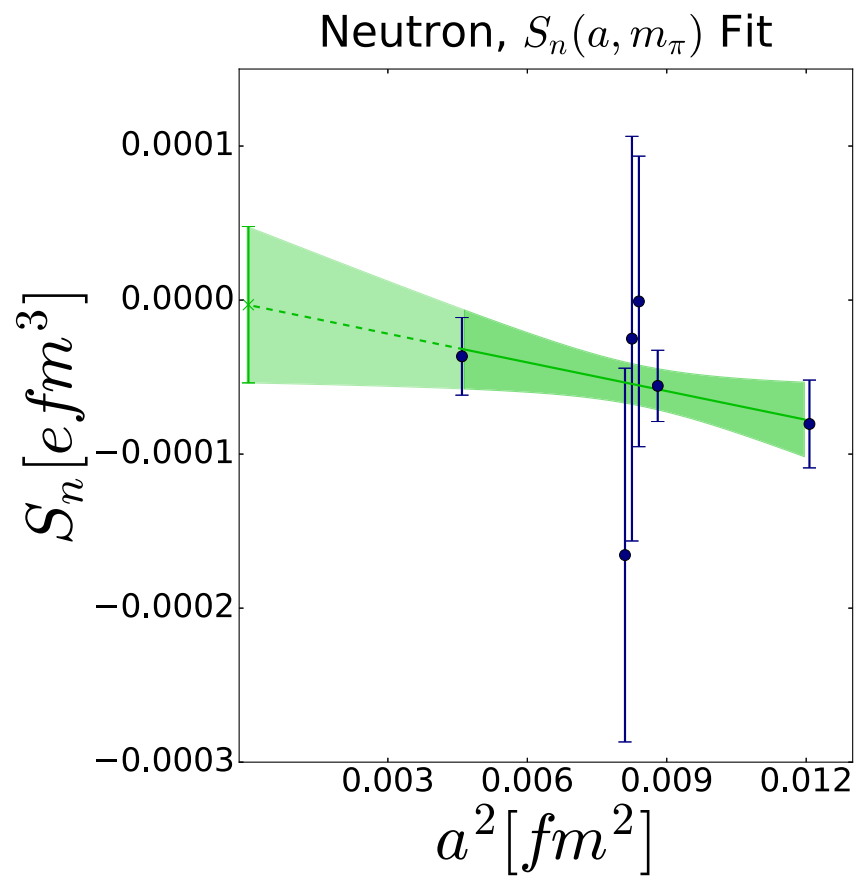

(a)

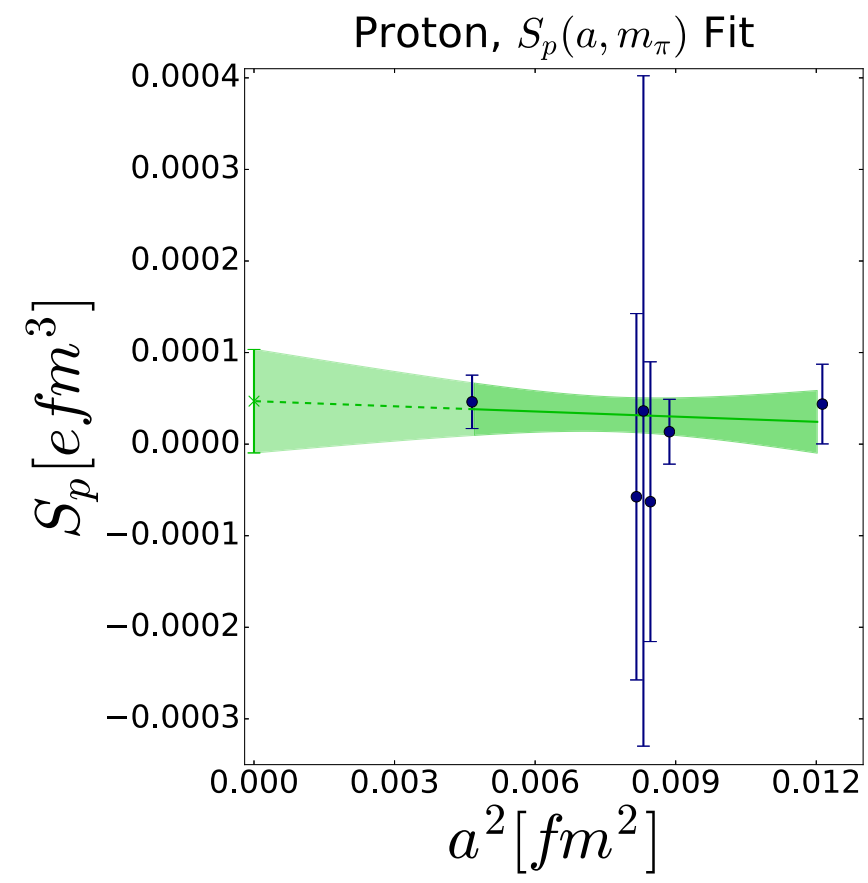

(b)

FIG. 26. Determination of the Schiff moment $S_{p / n}$ for the neutron (left) and proton (right) for all six of our ensembles, plotted against their respective lattice spacing values. The green band is the lattice spacing dependence of the fit to all the ensembles using Eq. (60). 
TABLE X. Neutron and proton Schiff fit parameters $C_{4}, C_{5}$ extracted from the combine fits to all six ensembles using Eq. (60), as well as the resulting $\chi_{\mathrm{PDF}}^{2}$. We additionally include the ratio $\frac{C_{2}}{C_{4}}$, where $C_{2}$ is the second fit parameter result from Table IX.

\begin{tabular}{lrrrr}
\hline \hline & $C_{4}\left[\bar{\theta} e \mathrm{fm}^{3}\right]$ & $C_{5}\left[\frac{\bar{\theta} e \mathrm{fm}^{3}}{\mathrm{fm}^{2}}\right]$ & $\chi_{\mathrm{PDF}}^{2}$ & \multicolumn{1}{c}{$\frac{C_{2}}{C_{4}} \approx-6$} \\
\hline Proton & $0.50(59) \times 10^{-4}$ & $-0.0022(73)$ & $1.25(80)$ & $-20(200)$ \\
Neutron & $-0.10(43) \times 10^{-4}$ & $-0.0057(51)$ & $1.37(97)$ & $70(970)$ \\
\hline \hline
\end{tabular}

perform a constant fit in the pion mass to obtain the proton and neutron Schiff moments at the physical point. We do not extrapolate to the chiral limit because the $\chi \mathrm{PT}$ prediction that $S_{p / n}$ are pion-mass independent will break down at some point as inferred from Eq. (56). We obtain for the Schiff moments at the physical point

$$
\begin{gathered}
S_{p}=0.50(59) \times 10^{-4} \bar{\theta} e \mathrm{fm}^{3}, \\
S_{n}=-0.10(43) \times 10^{-4} \bar{\theta} e \mathrm{fm}^{3},
\end{gathered}
$$

as well as the fit parameters $C_{4}=S_{p / n}$ and $C_{5}$ from performing this fit in Table $X$. The uncertainties are significant and the magnitudes are somewhat below the LO $\chi$ PT predictions in Eq. (51), but in better agreement once $\chi \mathrm{PT}$ NLO corrections are included. There is some evidence for a dominantly isovector Schiff moment as predicted from $\chi \mathrm{PT}$, but the uncertainties are too large to make strong statements. We perform a sanity check of our result by comparing the ChPT predictions for the fit coefficient $C_{2}$ and $C_{4}$. From Eqs. (43) and (51), we infer the LO ChPT prediction

$$
\frac{C_{2}}{C_{4}}=-6 \text {. }
$$

Our fit values for this ratio are given in Table $\mathrm{X}$, and agree with this prediction within (large) statistical errors.

\section{DISCUSSION}

In this section, we discuss the EDM and Schiff moment results for the neutron and proton. The succeeding Sec. VII A compares our determination of the EDM to previous lattice QCD EDM computations. Then following in Sec. VII B, the phenomenological ramifications of our results for the EDM and Schiff moments are discussed.

\section{A. Comparison with other works}

In this section, we compare the results obtained for the neutron EDM $d_{n}$ with few lattice QCD results from the literature. As noted in Ref. [6], it is sometimes problematic to compare different EDM calculations, as most results preceding this paper do not consider the rotation of the CP-odd form factor $F_{3}$ with $F_{2}$ and $\alpha_{N}$ computed on the lattice. ${ }^{4}$ The EDM $d_{n}$ (rotated to $F_{3}$ ) is shown in Fig. 27. Good agreement is seen from our

\footnotetext{
${ }^{4}$ We note that no general consensus has been reached about the need to perform this rotation of the form factors.
}

results to the others $[1,3]$ at $m_{\pi} \approx 475 \mathrm{MeV}$, but we see a slight tension between the results of $[3,4]$ and our results at $m_{\pi} \approx 350 \mathrm{MeV}$. It must be stressed that the rotation requires knowledge of the phase $\alpha_{N}$ and the unrotated form factors $F_{2}$ and $F_{3}$ which are not always easy to extract. To rotate the "C. Alexandrou et al., 2016" [4] results, an estimation of $F_{2}$ was determined from [51]. To rotate the "F.-K. Guo et al., 2015" [3] results, $F_{2}$ was determined from [52] (at $\left.\bar{\theta}=0\right)$ and $\alpha_{N}$ and $F_{3}$ estimated via a linear+cubic fit in $\bar{\theta}$ performed by Ref. [6].

In particular, the lattice results for $F_{3}$ not obtained in this work do not take into account correlations between $F_{2}, F_{3}$, and $\alpha_{N}$. As such, Fig. 27 is mainly shown for illustrative purposes and the error estimates for results not obtained in this work should be taken with a grain of salt.

\section{B. Impact on EDMs of light nuclei}

Armed with a nonperturbative determination of the nucleon EDMs as a function of $\bar{\theta}$ we can revisit EDMs of systems with more than a single nucleon. EDM experiments so far have mainly focused on neutral systems, but EDMs of charged particles can be probed if the particles are trapped in electromagnetic storage rings [53]. This technique has lead to a direct limit on the EDM of the muon [54], and to plans to pursue EDM measurements of protons and light nuclei in dedicated storage rings. Such measurements are still far away but impressive progress has been reported in Refs. [37,55]. EDMs of light nuclei have been calculated using chiral EFT $[25,26]$,

$$
\begin{aligned}
d_{{ }^{\mathrm{H}}}= & 0.94(1)\left(d_{n}+d_{p}\right)+\left[0.18(2) \bar{g}_{1}\right] e \mathrm{fm}, \\
d_{{ }^{3} \mathrm{H}}= & -0.03(1) d_{n}+0.92(1) d_{p} \\
& -\left[0.11(1) \bar{g}_{0}-0.14(2) \bar{g}_{1}\right] e \mathrm{fm}, \\
d_{{ }^{3} \mathrm{He}}= & 0.90(1) d_{n}-0.03(1) d_{p} \\
& +\left[0.11(1) \bar{g}_{0}+0.14(2) \bar{g}_{1}\right] e \mathrm{fm},
\end{aligned}
$$

in terms of the EDMs of nucleons and the CPV pion-nucleon coupling constants $\bar{g}_{0}$ and $\bar{g}_{1}$ associated to the interactions

$$
\mathcal{L}_{\pi N}(\bar{\theta})=\bar{g}_{0} \bar{N} \vec{\pi} \cdot \vec{\tau} N+\bar{g}_{1} \bar{N} \pi_{3} N .
$$

Values of $\bar{g}_{0}$ and $\bar{g}_{1}$ can be obtained from chiral-symmetry arguments $[34,56]$ that link these LECs to the hadron spectrum

$$
\begin{aligned}
& \bar{g}_{0}=-14.7(2.3) \times 10^{-3} \bar{\theta}, \\
& \bar{g}_{1}=3.4(2.4) \times 10^{-3} \bar{\theta},
\end{aligned}
$$

and the smallness of $\bar{g}_{1} / \bar{g}_{0}$ is due to approximate isospin symmetry.

In absence of direct lattice calculations of $d_{n}$ and $d_{p}$ we could only predict values for the combinations

$$
\begin{aligned}
d_{{ } \mathrm{H}}(\bar{\theta})-d_{n}(\bar{\theta})-d_{p}(\bar{\theta}) & =0.6(4) \times 10^{-3} \bar{\theta} e \mathrm{fm}, \\
d_{{ }^{3} \mathrm{H}}(\bar{\theta})-0.9 d_{p}(\bar{\theta}) & =2.1(5) \times 10^{-3} \bar{\theta} e \mathrm{fm}, \\
d_{{ }^{3} \mathrm{He}}(\bar{\theta})-0.9 d_{n}(\bar{\theta}) & =-1.1(5) \times 10^{-3} \bar{\theta} e \mathrm{fm} .
\end{aligned}
$$




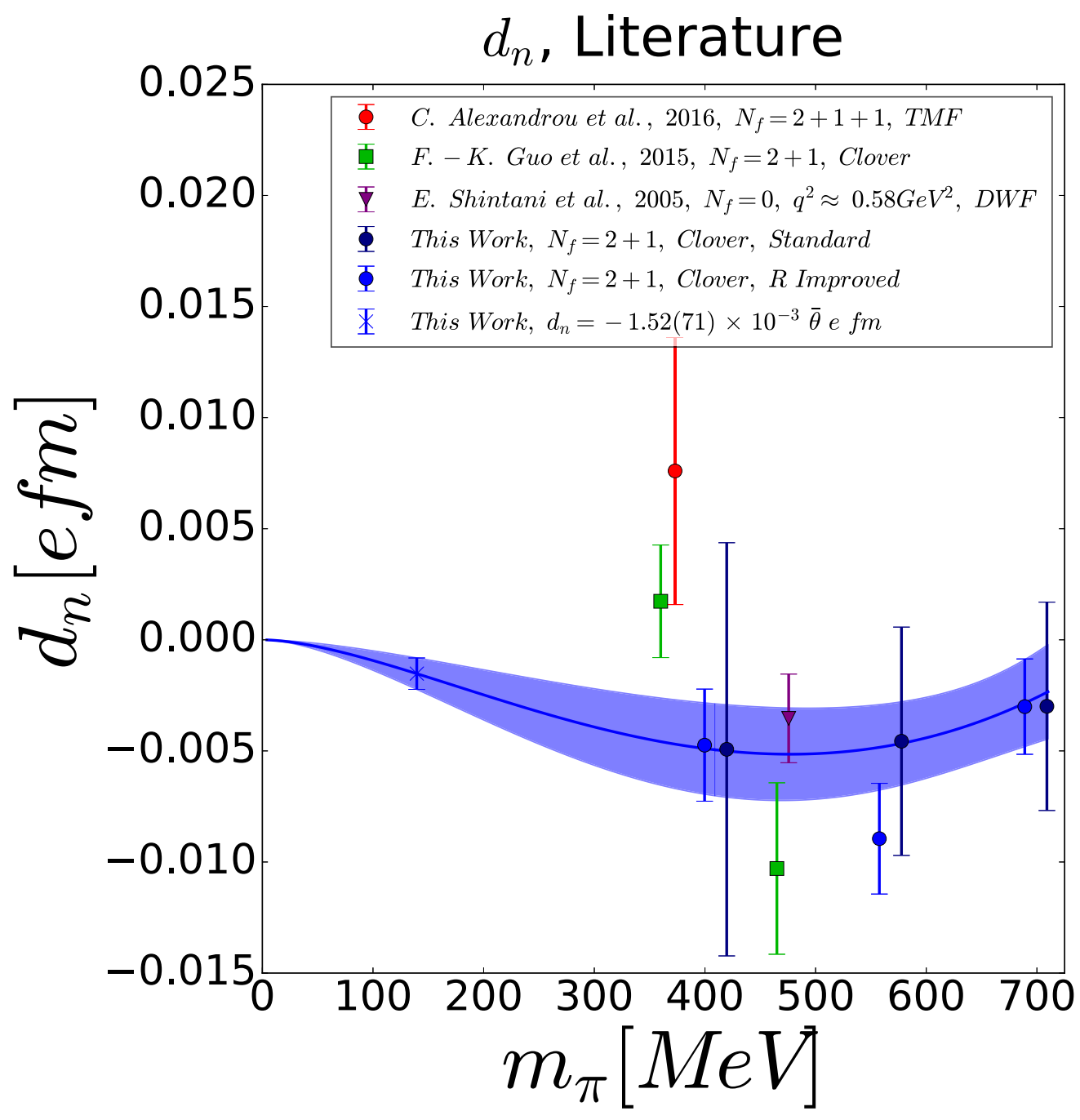

FIG. 27. The results of $d_{n}$ from this paper, improved in light blue and not-improved in dark blue, compared to other lattice QCD results $[1,3,4]$. The light blue bands correspond to a chiral extrapolation, using the improved data (light blue), after having performed the continuum limit as described in Sec. VIB. To perform the rotation of the $\mathrm{CP}$ odd form factor $F_{3}$ of other lattice calculations see the main text. We underline that our error determination for other works is purely illustrative since, not having at our disposal the raw data, we do not take into account correlations in the data.

But with our lattice determination of $d_{n}(\bar{\theta})$ and $d_{p}(\bar{\theta})$, we can now estimate the EDMs of light ions directly in terms of $\bar{\theta}$,

$$
\begin{aligned}
d_{{ }^{\mathrm{H}}}(\bar{\theta}) & =0.2(1.2) \times 10^{-3} \bar{\theta} e \mathrm{fm}, \\
d_{3_{\mathrm{H}}}(\bar{\theta}) & =3.2(1.0) \times 10^{-3} \bar{\theta} e \mathrm{fm}, \\
d_{3^{\mathrm{He}}}(\bar{\theta}) & =-2.5(0.8) \times 10^{-3} \bar{\theta} e \mathrm{fm} .
\end{aligned}
$$

Due to the dependence on the isoscalar nucleon EDM, $d_{n}+$ $d_{p}$, the deuteron EDM is still very uncertain. The tri-nucleon EDMs, however, are predicted more than three standard deviations from zero and with a fixed sign. The total uncertainty arises in roughly equal amounts from uncertainties in Eq. (68) and in the determination of the nucleon EDMs in Eq. (42). If nonzero EDMs are measured these relations can be used to differentiate between the SM $\theta$ term and BSM sources of $\mathrm{CP}$ violation. They can also provide indirect evidence for the existence of a Peccei-Quinn mechanism, by finding EDM patterns in disagreement with Eq. (70) [57].

\section{CONCLUSION}

In this paper we computed the proton and neutron EDM from dynamical lattice QCD using various pion masses at different lattice spacings and volumes, as enumerated in Tables I and II. We found our results have rather small (within our statistical uncertainties) discretization effects, which greatly simplified our continuum limit extrapolations. We found satisfactory agreement with existing results, as discussed in Table VII. With our measurements at multiple pion masses, we performed a chiral interpolation to obtain, at the physical pion mass and in the continuum limit $d_{n}=-0.00152(71) \bar{\theta} e \mathrm{fm}$ and $d_{p}=0.0011(10) \bar{\theta} e \mathrm{fm}$. The nonzero result for the neutron EDM confirms the existence 
of the strong $\mathrm{CP}$ problem at two standard deviations and limits $|\bar{\theta}|<1.98 \times 10^{-10}$ at $90 \%$ C.L. The dependence of the nucleon EDMs on the pion mass allowed us to extract the CP-odd pion-nucleon coupling, $\bar{g}_{0}$, and the resulting value is in good agreement with chiral perturbation theory. We have compared our chiral interpolation with a simple polynomial fit, and while the two interpolations give consistent results, the behavior of the expansion coefficients of the polynomial fit, and the consistency of the LEC $g_{0}$ obtained with two completely independent determination, gives us confidence that chiral perturbation theory describes the lattice data satisfactorily. More precise lattice data will allow for better tests.

Important to our analysis was the implementation of the gradient flow on the topological charge. In fact we can perform the continuum limit at fixed flow time with no need to calculate the normalization of the topological charge. As we have discussed and documented in Sec. V and ensuing subsections, the gradient flow also allows a more robust determination of the integrated autocorrelation time that must be taken into account when estimating statistical uncertainties.

Be that as it may, the extraction of a nonzero EDM is notoriously difficult due to its poor signal-to-noise ratio. To address this issue we have employed a novel technique, first documented in Ref. [12], for reducing the noise in our measured observables. Instead of summing all space-time points in the calculation of ratios relevant for the extraction of our 3-point function and $\alpha_{N}$ term, this method focuses on the space-time region where the signal is strongest. We argued that the neglected space-time region gives exponentially suppressed contributions to the correlation functions and this expectation has been confirmed by our numerical results. On some ensembles this method enabled us to increase the signal to noise by a factor of $\approx 2$. This method was described in detail in Sec. VI A and Appendix A.

We have also analysed the $Q^{2}$ dependence of our form factors and performed an extraction of the Schiff moment with dynamical fermions. Our results of $S_{p}=0.50(59) \times$ $10^{-4} \bar{\theta} \quad e \mathrm{fm}^{3}$ for the proton and $S_{n}=-0.10(43) \times$ $10^{-4} \bar{\theta} e \mathrm{fm}^{3}$ for the neutron are in reasonable agreement with chiral perturbation theory predictions. Our estimates for this value can be improved upon with more statistics and calculations on larger lattices (and thus lower $Q^{2}$ points), which would allow for a more robust extraction.

Our calculation represents a big step toward a precise determination of the nucleon EDM and Schiff moments. Improvement of these results will most definitely come from increased statistics, and more calculations at different pion masses at several lattice spacings. We comment here on the necessity to perform calculations at the physical pion mass. In the chiral limit the EDM induced by the $\theta$ term vanishes (i.e., $d_{p / n}=0$ at $m_{\pi}=0$ ). In our view, given the small value of the EDM induced by the $\theta$ term and the additional standard reduction in signal-to-noise as the pion mass is lowered for nucleon correlators, calculations of these quantities at the physical pion mass have possibly less to gain than those at higher pion masses. Because of this constraint, it could be advantageous to have results at slightly heavier-than-physical pion masses and then robustly interpolate to the physical pion mass using $\chi \mathrm{PT}$. The subsequent errors of the interpolation are stable and easily quantified precisely because one is doing an interpolation and not an extrapolation. We remark though that, for a chirally breaking lattice action, such as the nonperturbatively $\mathrm{O}(a)$ improved Wilson-clover fermion action we have adopted, the nucleon EDMs vanish in the chiral limit only after performing the continuum limit. This emphasizes the importance of the continuum limit when using a chirally breaking action. In this respect the gradient flow allows us to perform a safe study of discretization effects.

To summarize, the ideal scenario of a direct determination at the physical point with statistical uncertainties under control, can be circumvented by simply investing more time in lattice QCD calculations at slightly heavier pion masses (where the signal-to-noise is not as prohibitive). It goes without saying that calculations of the EDM at heavier-thanphysical pion masses can potentially be more cost effective than the physical-pion-mass calculations only if one has a robust description of the lattice data with $\chi \mathrm{PT}$.

\section{ACKNOWLEDGMENTS}

We thank Mattia Bruno, Taku Izubuchi, and Sergey Syritsyn for valuable discussions. This work was supported in part by Michigan State University through computational resources, provided by the Institute for Cyber-Enabled Research, and in part through financial support from the Deutsche Forschungsgemeinschaft (Sino-German CRC 110). The authors gratefully acknowledge the computing time granted through JARA-HPC on the supercomputer JURECA at Forschungszentrum Jülich. J.d.V. is supported by the RHIC Physics Fellow Program of the RIKEN BNL Research Center. A.S. acknowledges funding support under the National Science Foundation grant PHY-1913287.

\section{APPENDIX A: ALPHA IMPROVEMENT DERIVATION}

Staring with the general three-point correlation function

$$
\Delta_{2}^{(O)}\left(\mathbf{p}^{\prime}, t ; \mathbf{q}, \tau ; \Pi\right)=a^{6} \sum_{\mathbf{x}, \mathbf{y}} e^{-i \mathbf{p}^{\prime} \cdot(\mathbf{x}-\mathbf{y})} e^{i \mathbf{q} \cdot \mathbf{y}} \operatorname{Tr}\{\Pi\langle\mathcal{N}(\mathbf{x}, t) O(\mathbf{y}, \tau) \overline{\mathcal{N}}(\mathbf{0}, 0)\rangle\}
$$

we handle the time ordering in the next two sections, by performing the spectral decomposition for $t>\tau$ and $\tau<t$. This is the general expression for an arbitrary operator $O$ and spin projector $\Pi$. For the computation of the nucleon mixing angle $\alpha_{N}$, we have $O=\bar{Q}$. 


\section{Case $t>\tau$}

Starting with the specific time ordering $t>\tau$ in Eq. (A1), we perform the standard spectral decomposition to produce the correlation function

$$
\Delta_{2}^{(O)}\left(\mathbf{p}^{\prime}, t ; \mathbf{q}, \tau ; \Pi\right)=\sum_{\alpha, \beta, \gamma} \frac{1}{8 E_{\alpha} E_{\beta} E_{\gamma}} e^{-E_{\alpha}(T-t)} e^{-E_{\beta}(t-\tau)} e^{-E_{\gamma} \tau} \operatorname{Tr}\{\Pi\langle\alpha|\mathcal{N}| \beta\rangle\langle\beta|O| \gamma\rangle\langle\gamma|\overline{\mathcal{N}}| \alpha\rangle\},
$$

where the sum over states $\alpha, \beta, \gamma$ have been reduced to states that only contain momenta $\mathbf{p}_{\gamma}=\mathbf{q}, \mathbf{p}_{\beta}=\mathbf{p}-\mathbf{q}$, and $\mathbf{p}_{\alpha}=\mathbf{p}$. The two approximations one can apply to this equation are $T \gg t$ and $t \gg 0$, which are related to the source-sink separation of the two-point correlation function

$$
\Delta_{2}^{(O)}\left(\mathbf{p}^{\prime}, t ; \mathbf{q}, \tau ; \Pi\right)=\sum_{\beta, \gamma} \frac{1}{8 E_{\alpha_{0}} E_{\beta} E_{\gamma}} e^{-E_{\alpha_{0}}(T-t)} e^{-E_{\beta}(t-\tau)} e^{-E_{\gamma} \tau} \operatorname{Tr}\left\{\Pi\left\langle\alpha_{0}|\mathcal{N}| \beta\right\rangle\langle\beta|O| \gamma\rangle\left\langle\gamma|\overline{\mathcal{N}}| \alpha_{0}\right\rangle\right\},
$$

where $\alpha_{0}$ is the lowest lying energy state that gives a nonzero contribution to $\Delta_{2}^{(O)}$

$$
\operatorname{Tr}\left\{\Pi\left\langle\alpha_{0}|\mathcal{N}| \beta\right\rangle\langle\beta|O| \gamma\rangle\left\langle\gamma|\overline{\mathcal{N}}| \alpha_{0}\right\rangle\right\} \neq 0
$$

\section{Case $t<\tau$}

This has the same form, with replacing $\mathcal{N} \leftrightarrow O$

$$
\Delta_{2}^{(O)}\left(\mathbf{p}^{\prime}, t ; \mathbf{q}, \tau ; \Pi\right)=\sum_{\alpha, \beta, \gamma} \frac{1}{8 E_{\alpha} E_{\beta} E_{\gamma}} e^{-E_{\alpha}(T-\tau)} e^{-E_{\beta}(\tau-t)} e^{-E_{\gamma} t} \operatorname{Tr}\{\Pi\langle\alpha|O| \beta\rangle\langle\beta|\mathcal{N}| \gamma\rangle\langle\gamma|\overline{\mathcal{N}}| \alpha\rangle\}
$$

The two approximations $T \gg t$ and $t \gg 0$ are again applied

$$
\Delta_{2}^{(O)}\left(\mathbf{p}^{\prime}, t ; \mathbf{q}, \tau ; \Pi\right)=\sum_{\alpha, \beta} \frac{1}{8 E_{\alpha} E_{\beta} E_{\gamma_{0}}} e^{-E_{\alpha}(T-\tau)} e^{-E_{\beta}(\tau-t)} e^{-E_{\gamma_{0}} t} \operatorname{Tr}\left\{\Pi\langle\alpha|O| \beta\rangle\left\langle\beta|\mathcal{N}| \gamma_{0}\right\rangle\left\langle\gamma_{0}|\overline{\mathcal{N}}| \alpha\right\rangle\right\}
$$

where this time, $\gamma_{0}$ is the lowest lying state that gives a nonzero contribution to $\Delta_{2}^{(O)}$

$$
\operatorname{Tr}\left\{\Pi\langle\alpha|O| \beta\rangle\left\langle\beta|\mathcal{N}| \gamma_{0}\right\rangle\left\langle\gamma_{0}|\overline{\mathcal{N}}| \alpha\right\rangle\right\} \neq 0
$$

\section{Total form}

Over the total range $\tau \in[0, T]$, the expression is

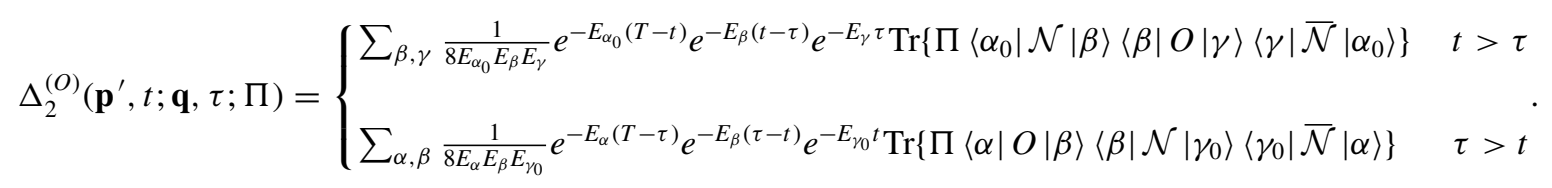

\section{Symmetric partially summed current $t_{s}>t$}

This region is where the fit will take place, so we omit the derivation for $t_{s}<t$.

$$
\begin{aligned}
\bar{G}_{2}^{(O)}\left(\mathbf{p}^{\prime}, t ; \mathbf{q}, t_{s} ; \Pi\right)= & a \sum_{\tau / a=0}^{t / a} \Delta_{2}^{(O)}\left(\mathbf{p}^{\prime}, t ; \mathbf{q}, \tau ; \Pi\right)+a \sum_{\tau / a=t / a+1}^{t_{s} / a} \Delta_{2}^{(O)}\left(\mathbf{p}^{\prime}, t ; \mathbf{q}, \tau ; \Pi\right)+a \sum_{\tau / a=0}^{t_{s} / a} \Delta_{2}^{(O)}\left(\mathbf{p}^{\prime}, t ; \mathbf{q}, T-\tau ; \Pi\right) \\
= & a \sum_{\tau / a=0}^{t / a} \sum_{\beta, \gamma} \frac{1}{8 E_{\alpha_{0}} E_{\beta} E_{\gamma}} e^{-E_{\alpha_{0}}(T-t)} e^{-E_{\beta}(t-\tau)} e^{-E_{\gamma} \tau} \operatorname{Tr}\left\{\Pi\left\langle\alpha_{0}|\mathcal{N}| \beta\right\rangle\langle\beta|O| \gamma\rangle\left\langle\gamma|\overline{\mathcal{N}}| \alpha_{0}\right\rangle\right\} \\
& +a \sum_{\tau / a=t / a+1}^{t_{s} / a} \sum_{\alpha, \beta} \frac{1}{8 E_{\alpha} E_{\beta} E_{\gamma_{0}}} e^{-E_{\alpha}(T-\tau)} e^{-E_{\beta}(\tau-t)} e^{-E_{\gamma_{0}} t} \operatorname{Tr}\left\{\Pi\langle\alpha|O| \beta\rangle\left\langle\beta|\mathcal{N}| \gamma_{0}\right\rangle\left\langle\gamma_{0}|\overline{\mathcal{N}}| \alpha\right\rangle\right\} \\
& +a \sum_{\tau / a=0}^{t_{s} / a} \sum_{\alpha, \beta} \frac{1}{8 E_{\alpha} E_{\beta} E_{\gamma_{0}}} e^{-E_{\alpha} \tau} e^{-E_{\beta}(T-t-\tau)} e^{-E_{\gamma_{0}} t} \operatorname{Tr}\left\{\Pi\langle\alpha|O| \beta\rangle\left\langle\beta|\mathcal{N}| \gamma_{0}\right\rangle\left\langle\gamma_{0}|\overline{\mathcal{N}}| \alpha\right\rangle\right\},
\end{aligned}
$$

noting the second sum is shifted to $\tau \in\left[t+a, t_{s}\right]$ using the lattice spacing increment $a$. 
One thing to note here, is the terms $\tau=0$ and $\tau=t$ are contact terms, which need to be handled properly (operator product expansion, or gradient flow). Next we group $\tau$ terms in preparation for the $t_{s}$ sum

$$
\begin{aligned}
\bar{G}_{2}^{(O)}\left(\mathbf{p}^{\prime}, t ; \mathbf{q}, t_{s} ; \Pi\right)= & \sum_{\beta, \gamma}\left[a \sum_{\tau / a=0}^{t / a} e^{-\left(E_{\gamma}-E_{\beta}\right) \tau}\right] \frac{1}{8 E_{\alpha_{0}} E_{\beta} E_{\gamma}} e^{-E_{\alpha_{0}}(T-t)} e^{-E_{\beta} t} \operatorname{Tr}\left\{\Pi\left\langle\alpha_{0}|\mathcal{N}| \beta\right\rangle\langle\beta|O| \gamma\rangle\left\langle\gamma|\overline{\mathcal{N}}| \alpha_{0}\right\rangle\right\} \\
& +\sum_{\alpha, \beta}\left[a \sum_{\tau / a=t / a+1}^{t_{s}} e^{-\left(E_{\beta}-E_{\alpha}\right) \tau}\right] \frac{1}{8 E_{\alpha} E_{\beta} E_{\gamma_{0}}} e^{-E_{\alpha} T} e^{-\left(E_{\gamma_{0}}-E_{\beta}\right) t} \operatorname{Tr}\left\{\Pi\langle\alpha|O| \beta\rangle\left\langle\beta|\mathcal{N}| \gamma_{0}\right\rangle\left\langle\gamma_{0}|\overline{\mathcal{N}}| \alpha\right\rangle\right\} \\
& +\sum_{\alpha, \beta}\left[a \sum_{\tau / a=0}^{t_{s} / a} e^{-\left(E_{\alpha}-E_{\beta}\right) \tau}\right] \frac{1}{8 E_{\alpha} E_{\beta} E_{\gamma_{0}}} e^{-E_{\beta}(T-t)} e^{-E_{\gamma_{0}} t} \operatorname{Tr}\left\{\Pi\langle\alpha|O| \beta\rangle\left\langle\beta|\mathcal{N}| \gamma_{0}\right\rangle\left\langle\gamma_{0}|\overline{\mathcal{N}}| \alpha\right\rangle\right\} .
\end{aligned}
$$

The sums can be computed by using

$$
\sum_{\tau / a=T_{0} / a}^{T / a} a e^{-E \tau}=\sum_{\tau / a=T_{0} / a}^{T / a} a e^{-E a(\tau / a)}=a \frac{e^{E-E T_{0}}-e^{-E T}}{e^{E}-1}=a \frac{e^{E\left(1-T_{0}\right)}-e^{-E T}}{e^{E}-1},
$$

substituting in this expression, and including the terms where $\alpha=\beta$ and $\gamma=\beta$ separately,

$$
\begin{aligned}
\bar{G}_{2}^{(O)}\left(\mathbf{p}^{\prime}, t ; \mathbf{q}, t_{s} ; \Pi\right)= & \sum_{\beta} \frac{t}{8 E_{\alpha_{0}} E_{\beta}^{2}} e^{-E_{\alpha_{0}}(T-t)} e^{-E_{\beta} t} \operatorname{Tr}\left\{\Pi\left\langle\alpha_{0}|\mathcal{N}| \beta\right\rangle\langle\beta|O| \beta\rangle\left\langle\beta|\overline{\mathcal{N}}| \alpha_{0}\right\rangle\right\} \\
& +\sum_{\alpha} \frac{2 t_{s}-t-a}{8 E_{\alpha}^{2} E_{\gamma_{0}}} e^{-E_{\alpha}(T-t)} e^{-E_{\gamma_{0}} t} \operatorname{Tr}\left\{\Pi\langle\alpha|O| \alpha\rangle\left\langle\alpha|\mathcal{N}| \gamma_{0}\right\rangle\left\langle\gamma_{0}|\overline{\mathcal{N}}| \alpha\right\rangle\right\} \\
& +a \sum_{\beta \neq \gamma} \frac{e^{\left(E_{\gamma}-E_{\beta}\right) a}-e^{-\left(E_{\gamma}-E_{\beta}\right) t}}{8\left[e^{\left(E_{\gamma}-E_{\beta}\right) a}-1\right] E_{\alpha_{0}} E_{\beta} E_{\gamma}} e^{-E_{\alpha_{0}}(T-t)} e^{-E_{\beta} t} \operatorname{Tr}\left\{\Pi\left\langle\alpha_{0}|\mathcal{N}| \beta\right\rangle\langle\beta|O| \gamma\rangle\left\langle\gamma|\overline{\mathcal{N}}| \alpha_{0}\right\rangle\right\} \\
& +a \sum_{\alpha \neq \beta} \frac{e^{\left(E_{\beta}-E_{\alpha}\right) t}-e^{-\left(E_{\beta}-E_{\alpha}\right) t_{s}}}{8\left[e^{\left(E_{\beta}-E_{\alpha}\right) a}-1\right] E_{\alpha} E_{\beta} E_{\gamma_{0}}} e^{-E_{\alpha} T} e^{-\left(E_{\gamma_{0}}-E_{\beta}\right) t} \operatorname{Tr}\left\{\Pi\langle\alpha|O| \beta\rangle\left\langle\beta|\mathcal{N}| \gamma_{0}\right\rangle\left\langle\gamma_{0}|\overline{\mathcal{N}}| \alpha\right\rangle\right\} \\
& +a \sum_{\alpha \neq \beta} \frac{e^{\left(E_{\alpha}-E_{\beta}\right) t}-e^{-\left(E_{\alpha}-E_{\beta}\right) t_{s}}}{8\left[e^{\left(E_{\alpha}-E_{\beta}\right) a}-1\right] E_{\alpha} E_{\beta} E_{\gamma_{0}}} e^{-E_{\beta}(T-t)} e^{-E_{\gamma_{0}} t} \operatorname{Tr}\left\{\Pi\langle\alpha|O| \beta\rangle\left\langle\beta|\mathcal{N}| \gamma_{0}\right\rangle\left\langle\gamma_{0}|\overline{\mathcal{N}}| \alpha\right\rangle\right\} .
\end{aligned}
$$

As the final two terms are only exponentially dependent on $t_{s}$, we write these terms as exponentials of single energy indices

$$
\begin{aligned}
\bar{G}_{2}^{(O)}\left(\mathbf{p}^{\prime}, t ; \mathbf{q}, t_{s} ; \Pi\right)= & \sum_{\beta} \frac{t}{8 E_{\alpha_{0}} E_{\beta}^{2}} e^{-E_{\alpha_{0}}(T-t)} e^{-E_{\beta} t} \operatorname{Tr}\left\{\Pi\left\langle\alpha_{0}|\mathcal{N}| \beta\right\rangle\langle\beta|O| \beta\rangle\left\langle\beta|\overline{\mathcal{N}}| \alpha_{0}\right\rangle\right\} \\
& +\sum_{\alpha} \frac{2 t_{s}-t-a}{8 E_{\alpha}^{2} E_{\gamma_{0}}} e^{-E_{\alpha}(T-t)} e^{-E_{\gamma_{0}} t} \operatorname{Tr}\left\{\Pi\langle\alpha|O| \alpha\rangle\left\langle\alpha|\mathcal{N}| \gamma_{0}\right\rangle\left\langle\gamma_{0}|\overline{\mathcal{N}}| \alpha\right\rangle\right\} \\
& +a \sum_{\beta \neq \gamma} \frac{e^{\left(E_{\gamma}-E_{\beta}\right) a}-e^{-\left(E_{\gamma}-E_{\beta}\right) t}}{8\left[e^{\left(E_{\gamma}-E_{\beta}\right) a}-1\right] E_{\alpha_{0}} E_{\beta} E_{\gamma}} e^{-E_{\alpha_{0}}(T-t)} e^{-E_{\beta} t} \operatorname{Tr}\left\{\Pi\left\langle\alpha_{0}|\mathcal{N}| \beta\right\rangle\langle\beta|O| \gamma\rangle\left\langle\gamma|\overline{\mathcal{N}}| \alpha_{0}\right\rangle\right\} \\
& +a \sum_{\alpha \neq \beta} \frac{e^{-E_{\alpha}(T+t)} e^{-\left(E_{\gamma_{0}}-2 E_{\beta}\right) t}-e^{-E_{\alpha}\left(T-t_{s}\right)} e^{-E_{\gamma_{0}} t} e^{-E_{\beta}\left(t_{s}-t\right)}}{8\left[e^{\left(E_{\beta}-E_{\alpha}\right) a}-1\right] E_{\alpha} E_{\beta} E_{\gamma_{0}}} \operatorname{Tr}\left\{\Pi\langle\alpha|O| \beta\rangle\left\langle\beta|\mathcal{N}| \gamma_{0}\right\rangle\left\langle\gamma_{0}|\overline{\mathcal{N}}| \alpha\right\rangle\right\} \\
& +a \sum_{\alpha \neq \beta} \frac{e^{-E_{\beta} T} e^{-E_{\gamma_{0}} t e^{E_{\alpha} t}-e^{-E_{\beta}\left(T-t-t_{s}\right)} e^{-E_{\gamma_{0}} t} e^{-E_{\alpha} t_{s}}}}{8\left[e^{\left(E_{\alpha}-E_{\beta}\right) a}-1\right] E_{\alpha} E_{\beta} E_{\gamma_{0}}} \operatorname{Tr}\left\{\Pi\langle\alpha|O| \beta\rangle\left\langle\beta|\mathcal{N}| \gamma_{0}\right\rangle\left\langle\gamma_{0}|\overline{\mathcal{N}}| \alpha\right\rangle\right\},
\end{aligned}
$$

and clumping like terms

$$
\begin{aligned}
\bar{G}_{2}^{(O)}\left(\mathbf{p}^{\prime}, t ; \mathbf{q}, t_{s} ; \Pi\right)= & \sum_{\beta} \frac{t}{8 E_{\alpha_{0}} E_{\beta}^{2}} e^{-E_{\alpha_{0}}(T-t)} e^{-E_{\beta} t} \operatorname{Tr}\left\{\Pi\left\langle\alpha_{0}|\mathcal{N}| \beta\right\rangle\langle\beta|O| \beta\rangle\left\langle\beta|\overline{\mathcal{N}}| \alpha_{0}\right\rangle\right\} \\
& +\sum_{\alpha} \frac{2 t_{s}-t-a}{8 E_{\alpha}^{2} E_{\gamma_{0}}} e^{-E_{\alpha}(T-t)} e^{-E_{\gamma_{0}} t} \operatorname{Tr}\left\{\Pi\langle\alpha|O| \alpha\rangle\left\langle\alpha|\mathcal{N}| \gamma_{0}\right\rangle\left\langle\gamma_{0}|\overline{\mathcal{N}}| \alpha\right\rangle\right\} \\
& +a \sum_{\beta \neq \gamma} \frac{e^{\left(E_{\gamma}-E_{\beta}\right) a}-e^{-\left(E_{\gamma}-E_{\beta}\right) t}}{8\left[e^{\left(E_{\gamma}-E_{\beta}\right) a}-1\right] E_{\alpha_{0}} E_{\beta} E_{\gamma}} e^{-E_{\alpha_{0}}(T-t)} e^{-E_{\beta} t} \operatorname{Tr}\left\{\Pi\left\langle\alpha_{0}|\mathcal{N}| \beta\right\rangle\langle\beta|O| \gamma\rangle\left\langle\gamma|\overline{\mathcal{N}}| \alpha_{0}\right\rangle\right\}
\end{aligned}
$$




$$
\begin{aligned}
& +a \sum_{\alpha \neq \beta} \operatorname{Tr}\left\{\Pi\langle\alpha|O| \beta\rangle\left\langle\beta|\mathcal{N}| \gamma_{0}\right\rangle\left\langle\gamma_{0}|\overline{\mathcal{N}}| \alpha\right\rangle\right\} e^{-E_{\gamma_{0}} t} \\
& \times\left[\frac{e^{-E_{\alpha}(T+t)} e^{2 E_{\beta} t}-e^{-E_{\alpha}\left(T-t_{s}\right)} e^{-E_{\beta}\left(t_{s}-t\right)}}{8\left[e^{\left(E_{\beta}-E_{\alpha}\right) a}-1\right] E_{\alpha} E_{\beta} E_{\gamma_{0}}}+\frac{e^{-E_{\beta} T} e^{E_{\alpha} t}-e^{-E_{\beta}\left(T-t-t_{s}\right)} e^{-E_{\alpha} t_{s}}}{8\left[e^{\left(E_{\alpha}-E_{\beta}\right) a}-1\right] E_{\alpha} E_{\beta} E_{\gamma_{0}}}\right] .
\end{aligned}
$$

\section{Explicit form for $O=\bar{Q}$}

As $\bar{Q}$ is a parity violating operator, the nucleon states that propagate before and after this operator must be opposite in parity. This removes the first and second terms as $\langle\beta|\bar{Q}| \beta\rangle=0$. As well as this, the terms with sums over two terms either require $\left(\alpha, \beta=\alpha_{+}, \beta_{-}\right)$or $\left(\alpha, \beta=\alpha_{-}, \beta_{+}\right)$where the subscript \pm refers to the state having positive or negative parity. The projector is selected to be $\Pi=\gamma_{5} \Pi_{+}=\gamma_{5} \frac{I+\gamma_{4}}{2}$, which results in only the trace term with $\left(\alpha, \beta=\alpha_{+}, \beta_{-}\right)$being nonzero

$$
\begin{aligned}
\bar{G}_{2}^{(\bar{Q})}\left(\mathbf{p}^{\prime}, t ; \mathbf{q}, t_{s} ; \gamma_{5} \Pi_{+}\right)= & a \sum_{\beta \neq \gamma} \frac{e^{\left(E_{\gamma}-E_{\beta}\right) a}-e^{-\left(E_{\gamma}-E_{\beta}\right) t}}{8\left[e^{\left(E_{\gamma}-E_{\beta}\right) a}-1\right] E_{\alpha_{0}} E_{\beta} E_{\gamma}} e^{-E_{\alpha_{0}}(T-t)} e^{-E_{\beta} t} \operatorname{Tr}\left\{\gamma_{5} \Pi_{+}\left\langle\alpha_{0}|\mathcal{N}| \beta\right\rangle\langle\beta|\bar{Q}| \gamma\rangle\left\langle\gamma|\overline{\mathcal{N}}| \alpha_{0}\right\rangle\right\} \\
& +a \sum_{\alpha_{+}, \beta_{-}} \operatorname{Tr}\left\{\gamma_{5} \Pi_{+}\left\langle\alpha_{+}|\bar{Q}| \beta_{-}\right\rangle\left\langle\beta_{-}|\mathcal{N}| \gamma_{0}\right\rangle\left\langle\gamma_{0}|\overline{\mathcal{N}}| \alpha_{+}\right\rangle\right\} e^{-E_{\gamma_{0}} t} \\
& \times\left[\frac{e^{-E_{\alpha_{+}}(T+t)} e^{2 E_{\beta_{-}} t}-e^{-E_{\alpha_{+}}\left(T-t_{s}\right)} e^{-E_{\beta_{-}}\left(t_{s}-t\right)}}{8\left[e^{\left(E_{\beta_{-}}-E_{\alpha_{+}}\right) a}-1\right] E_{\alpha_{+}} E_{\beta_{-}} E_{\gamma_{0}}}+\frac{e^{-E_{\beta_{-}} T} e^{E_{\alpha_{+}} t}-e^{-E_{\beta_{-}}\left(T-t-t_{s}\right)} e^{-E_{\alpha_{+} t_{s}}}}{8\left[e^{\left(E_{\alpha_{+}}-E_{\beta_{-}}\right) a}-1\right] E_{\alpha_{+}} E_{\beta_{-}} E_{\gamma_{0}}}\right] .
\end{aligned}
$$

The terms $e^{-E_{\alpha_{+}}(T+t)}$ and $e^{-E_{\alpha_{+}}\left(T-t_{s}\right)}$ in the final sum are exponentially suppressed as $T \gg T / 2 \geqslant t_{s}$ and $T \gg t$

$$
\begin{aligned}
\bar{G}_{2}^{(\bar{Q})}\left(\mathbf{p}^{\prime}, t ; \mathbf{q}, t_{s} ; \gamma_{5} \Pi_{+}\right)= & a \sum_{\beta \neq \gamma} \frac{e^{\left(E_{\gamma}-E_{\beta}\right) a}-e^{-\left(E_{\gamma}-E_{\beta}\right) t}}{8\left[e^{\left(E_{\gamma}-E_{\beta}\right) a}-1\right] E_{\alpha_{0}} E_{\beta} E_{\gamma}} e^{-E_{\alpha_{0}}(T-t)} e^{-E_{\beta} t} \operatorname{Tr}\left\{\gamma_{5} \Pi_{+}\left\langle\alpha_{0}|\mathcal{N}| \beta\right\rangle\langle\beta|\bar{Q}| \gamma\rangle\left\langle\gamma|\overline{\mathcal{N}}| \alpha_{0}\right\rangle\right\} \\
& +a \sum_{\alpha_{+}, \beta_{-}} \frac{e^{-E_{\beta_{-}} T} e^{E_{\alpha_{+}} t}}{8\left[e^{\left(E_{\alpha_{+}}-E_{\beta_{-}}\right) a}-1\right] E_{\alpha_{+}} E_{\beta_{-}} E_{\gamma_{0}}} e^{-E_{\gamma_{0} t} t} \operatorname{Tr}\left\{\gamma_{5} \Pi_{+}\left\langle\alpha_{+}|\bar{Q}| \beta_{-}\right\rangle\left\langle\beta_{-}|\mathcal{N}| \gamma_{0}\right\rangle\left\langle\gamma_{0}|\overline{\mathcal{N}}| \alpha_{+}\right\rangle\right\} \\
& -a \sum_{\alpha_{+}, \beta_{-}} \frac{e^{-E_{\beta_{-}}\left(T-t-t_{s}\right)} e^{-E_{\alpha_{+}} t_{s}}}{8\left[e^{\left(E_{\alpha_{+}}-E_{\beta_{-}}\right) a}-1\right] E_{\alpha_{+}} E_{\beta_{-}} E_{\gamma_{0}}} e^{-E_{\gamma_{0} t} t} \operatorname{Tr}\left\{\gamma_{5} \Pi_{+}\left\langle\alpha_{+}|\bar{Q}| \beta_{-}\right\rangle\left\langle\beta_{-}|\mathcal{N}| \gamma_{0}\right\rangle\left\langle\gamma_{0}|\overline{\mathcal{N}}| \alpha_{+}\right\rangle\right\}
\end{aligned}
$$

From this complicated expression, the $t_{s}$ dependence only appears exponentially in the final term. Therefore, we can fit the two-point correlation function with

$$
\text { fit }\left(t_{s}\right)=A+B e^{-E t_{s}} \text {. }
$$

Due to the statistical noise of the data and high correlation in the data with respect to $t_{s}$, we elected to neglect the excited state term by fitting a constant in the region where $B e^{-E t_{s}} \ll$ A.

\section{APPENDIX B: RATIO FUNCTION FIT RANGE SELECTION}

In this Appendix, we present the technique used for extracting the CP-odd form factor $F_{3}\left(Q^{2}\right)$ from the ratio function in Eq. (34). Since only constant ("one-state") fits are implemented for the ratio functions, careful consideration to excited state effects is needed.

The method employed to account for fit range dependence in our error estimates, is to include multiple fit ranges that satisfy some $\chi^{2}$ per degree of freedom $\left(\chi_{\mathrm{PDF}}^{2}\right)$ criterium. For this study, we only select fits that satisfy $\chi_{\mathrm{PDF}}^{2} \in[0.5,1]$. Using the multiple fit range determinations of $R$ and $R^{Q}$, we extend
Eq. (35) to include different fit ranges:

$$
\sum_{i=1}^{3} \mathcal{A}\left(Q^{2}\right)_{A i} F_{i, f(A)}\left(Q^{2}\right)=\left\{\begin{array}{l}
R_{f(A)}\left(\mathbf{0}, t, \boldsymbol{q}_{j}, \Pi_{k}, \gamma_{l}\right) \\
R_{f(A)}^{(Q)}\left(\mathbf{0}, t, \boldsymbol{q}_{j}, \Pi_{k}, \gamma_{l}, t_{f}\right)
\end{array},\right.
$$

where the extra index $f(A)$ refers to which fit range is used, which depends on the collective index $A=\{j, k, l\}, A \in$ $\left[1, \ldots, N_{A}\right]$.

Since each ratio function selected by index $A$ has $f(A)$ different ways to extract the quantity, the system is solved for every combination of $f(A) \forall A \in\left[1, \ldots, N_{A}\right]$. This results in $\prod_{A} F(A)$ independent system of equations to solve, where $F(A)$ is the number of different fits accepted (using the $\chi_{\mathrm{PDF}}^{2}$ criterium) for index $f(A)=1,2, \ldots, F(A)$.

Once the form factors have been solved over different fit range combinations, the result we obtain is $F_{i, f}\left(Q^{2}\right)$, where the (A missing) index $f$ refers to which combined set of fit ranges were used. Since the extrapolation to $Q^{2} \rightarrow 0$ must be performed to compute the nucleon EDM, this must be performed for every $f\left(Q^{2}\right)$ combination (analogous to $f(A)$ above). So, in addition to the above, we increase the number of fits to $\prod_{Q^{2}} F\left(Q^{2}\right)$, where $F\left(Q^{2}\right)$ is the number of fits computed for index $f\left(Q^{2}\right)=1,2, \ldots, F\left(Q^{2}\right)$. 


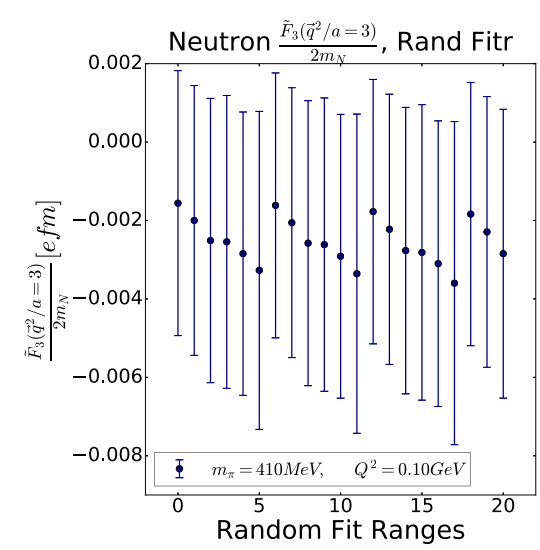

(a)

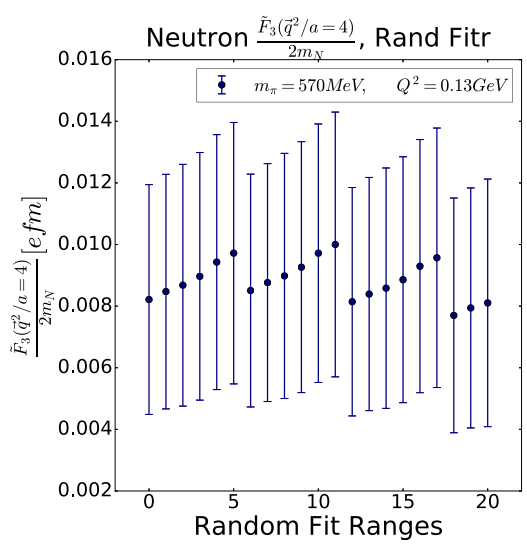

(b)

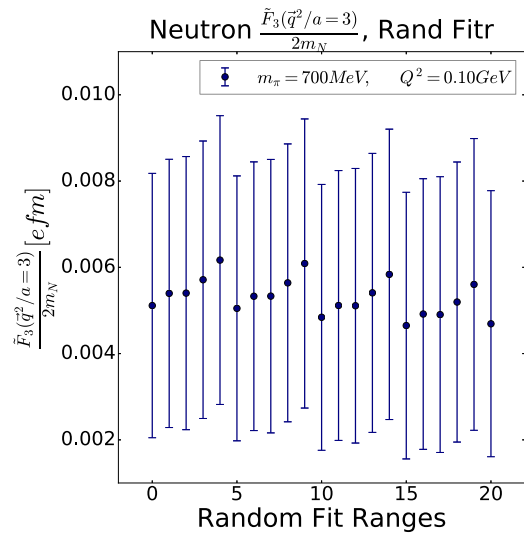

(c)

FIG. 28. Comparison of different randomly selected fit ranges used in the solving of the CP-odd form factor $\frac{F_{3}\left(Q^{2}\right)}{2 M_{N}}$ using the $m_{\pi}=$ $410,570,700 \mathrm{MeV}$ (left, middle, and right) ensembles. Although lattice transfer momentum increment $(\boldsymbol{q} / a)^{2}=3,4$ were selected, all other momenta exhibited the same (lack of) behavior.

Combining both these studies together, the resulting nucleon EDM has been computed using different fit ranges, indexed by $\frac{F_{3, f}^{p / n}\left(Q^{2} \rightarrow 0\right)}{2 M_{N}}=d_{p / n, f}$. So to obtain a final result where the statistical uncertainty from the gauge fields and the systematic errors arising from the fit ranges can be combine into a single uncertainty, we extend the bootstrap samples which are already used to compute the statistical uncertainty $d_{p / n, B}$ where $B$ runs over $\left[1, N_{b}\right] \otimes\left[1, N_{F}\right]$ where $N_{f}$ is the number of fits which each have $N_{b}$ bootstrap samples.

\section{Computational viability}

As one may notice, the above formulation is of order $O(A !)$, assuming a fixed number of fit ranges selected. A stochastic estimation of the fit range variation is highly recommended, which can be employed when solving the form factor Eq. (B1), as well as when taking the form factor $Q^{2} \rightarrow 0$ extrapolation.

At the form factor solving stage, this is employed by randomly selecting $N_{\chi}$ different fit range that satisfy the $\chi_{\mathrm{PDF}}^{2}$ criterium. The resulting number of systems of equations to be solved are $N_{\chi}^{N_{A}}$.
For the form factor extrapolation in $Q^{2} \rightarrow 0$, a random selection of $N_{F}$ results of index $f\left(Q^{2}\right)$ in $F_{N, f\left(Q^{2}\right)}\left(Q^{2}\right)$. The resulting number of fits to be performed using this estimation is $N_{F}^{N_{Q}}$ for $N_{Q}$ number of transfer momentum $Q^{2}$.

\section{Results computed in this paper}

The results computed in this paper use the fit criterium $\chi_{\mathrm{PDF}}^{2} \in[0.5,1]$, and excluded fits of length 2 . The cutoff for the number of fit ranges per ratio function is $N_{\chi}=4$ and the cutoff for the form factor extrapolation is $N_{Q}=4$ as well.

As for the number of equations to solve, results at lattice $(\boldsymbol{q} / a)^{2}=1,4$ has 1024 equations, $(\boldsymbol{q} / a)^{2}=2$ has 4096 equations and $(\boldsymbol{q} / a)^{2}=3$ has 16384 equations. Multiplying these numbers by 200 bootstrap samples, will give the individual number of system of equations solved. Once this is complete, we avoid computing $\sim 70$ trillion equations by performing the stochastic estimate which only requires 256 equations to solve for. Although it may seem the values for $N_{\chi}$ and $N_{F}$ are insufficient in size, the results shown in Fig. 28 demonstrates minimal variation when analyzing each individual $\frac{F_{3, f}^{p / n}\left(Q^{2}\right)}{2 M_{N}}$ over different fit ranges $f$.
[1] E. Shintani et al., Phys. Rev. D 72, 014504 (2005).

[2] F. Berruto, T. Blum, K. Orginos, and A. Soni, Phys. Rev. D 73, 054509 (2006).

[3] F. K. Guo, R. Horsley, U. G. Meissner, Y. Nakamura, H. Perlt, P. E. L. Rakow, G. Schierholz, A. Schiller, and J. M. Zanotti, Phys. Rev. Lett. 115, 062001 (2015).

[4] C. Alexandrou, A. Athenodorou, M. Constantinou, K. Hadjiyiannakou, K. Jansen, G. Koutsou, K. Ottnad, and M. Petschlies, Phys. Rev. D 93, 074503 (2016).

[5] E. Shintani, T. Blum, T. Izubuchi, and A. Soni, Phys. Rev. D 93, 094503 (2016).
[6] M. Abramczyk, S. Aoki, T. Blum, T. Izubuchi, H. Ohki, and S. Syritsyn, Phys. Rev. D 96, 014501 (2017).

[7] B. Yoon, T. Bhattacharya, and R. Gupta, EPJ Web Conf. 175, 01014 (2018).

[8] T. Bhattacharya, V. Cirigliano, R. Gupta, E. Mereghetti, and B. Yoon, Phys. Rev. D 92, 114026 (2015).

[9] M. Constantinou, EPJ Web Conf. 137, 08003 (2017).

[10] A. Shindler, J. de Vries, and T. Luu, PoS (LATTICE2014), 251 (2014).

[11] A. Shindler, T. Luu, and J. de Vries, Phys. Rev. D 92, 094518 (2015). 
[12] J. Dragos, T. Luu, A. Shindler, J. de Vries, and A. Yousif, PoS (LATTICE2018), 259 (2019).

[13] M. D. Rizik, C. Monahan, and A. Shindler, PoS (LATTICE2018), 215 (2018).

[14] J. Kim, J. Dragos, A. Shindler, T. Luu, and J. de Vries, PoS (LATTICE2018), 260 (2019).

[15] J. G. Reyes, J. Dragos, J. Kim, A. Shindler, and T. Luu, PoS (LATTICE2018), 219 (2018).

[16] M. D. Rizik, C. J. Monahan, and A. Shindler (SymLat Collaboration), Phys. Rev. D 102, 034509 (2020).

[17] M. Lüscher, J. High Energy Phys. 08 (2010) 071.

[18] M. Lüscher and P. Weisz, J. High Energy Phys. 02 (2011) 051.

[19] M. Cè, C. Consonni, G. P. Engel, and L. Giusti, Phys. Rev. D 92, 074502 (2015).

[20] R. Crewther, P. Di Vecchia, G. Veneziano, and E. Witten, Phys. Lett. B 88, 123 (1979).

[21] K. Ottnad, B. Kubis, U.-G. Meißner, and F.-K. Guo, Phys. Lett. B 687, 42 (2010).

[22] J. de Vries, R. G. E. Timmermans, E. Mereghetti, and U. van Kolck, Phys. Lett. B 695, 268 (2011).

[23] E. Mereghetti, J. de Vries, W. Hockings, C. Maekawa, and U. van Kolck, Phys. Lett. B 696, 97 (2011).

[24] F.-K. Guo and U.-G. Meißner, J. High Energy Phys. 12 (2012) 097.

[25] J. de Vries, R. Higa, C. P. Liu, E. Mereghetti, I. Stetcu, R. G. E. Timmermans, and U. van Kolck, Phys. Rev. C 84, 065501 (2011).

[26] J. Bsaisou et al., J. High Energy Phys. 03 (2015) 104; 05 (2015) 083(E).

[27] V. Baluni, Phys. Rev. D 19, 2227 (1979).

[28] E. M. Purcell and N. F. Ramsey, Phys. Rev. 78, 807 (1950).

[29] J. H. Smith, E. M. Purcell, and N. F. Ramsey, Phys. Rev. 108, 120 (1957).

[30] C. Baker et al., Phys. Rev. Lett. 97, 131801 (2006).

[31] J. Pendlebury et al., Phys. Rev. D 92, 092003 (2015).

[32] T. E. Chupp, P. Fierlinger, M. J. Ramsey-Musolf, and J. T. Singh, Rev. Mod. Phys. 91, 015001 (2019).

[33] E. Mereghetti, W. H. Hockings, and U. van Kolck, Ann. Phys. 325, 2363 (2010).

[34] J. de Vries, E. Mereghetti, and A. Walker-Loud, Phys. Rev. C 92, 045201 (2015).

[35] S. Borsanyi et al., Science 347, 1452 (2015).

[36] D. A. Brantley, B. Joo, E. V. Mastropas, E. Mereghetti, H. Monge-Camacho, B. C. Tiburzi, and A. Walker-Loud, arXiv:1612.07733.
[37] D. Eversmann et al. (JEDI Collaboration), Phys. Rev. Lett. 115, 094801 (2015).

[38] M. G. Beckett et al., Comput. Phys. Commun. 182, 1208 (2011).

[39] S. Aoki, K. I. Ishikawa, N. Ishizuka, T. Izubuchi, D. Kadoh, K. Kanaya, Y. Kuramashi, Y. Namekawa, M. Okawa, Y. Taniguchi, A. Ukawa, N. Ukita, and T. Yoshie, Phys. Rev. D 79, 034503 (2009).

[40] T. Ishikawa, S. Aoki, M. Fukugita, S. Hashimoto, K. I. Ishikawa, N. Ishizuka, Y. Iwasaki, K. Kanaya, T. Kaneko, Y. Kuramashi, M. Okawa, Y. Taniguchi, N. Tsutsui, A. Ukawa, N. Yamada, and T. Yoshie, Phys. Rev. D 78, 011502(R) (2008).

[41] S. Gusken, Nucl. Phys. Proc. Suppl. 17, 361 (1990).

[42] C. Alexandrou, F. Jegerlehner, S. Gusken, K. Schilling, and R. Sommer, Phys. Lett. B 256, 60 (1991).

[43] S. Aoki et al. (PACS-CS Collaboration), J. High Energy Phys. 08 (2010) 101.

[44] S. O. Bilson-Thompson, D. B. Leinweber, and A. G. Williams, Ann. Phys. 304, 1 (2003).

[45] U. Wolff (ALPHA Collaboration), Comput. Phys. Commun. 156, 143 (2004); 176, 383(E) (2007).

[46] M. Lüscher and S. Schaefer, J. High Energy Phys. 07 (2011) 036.

[47] M. Bruno, S. Schaefer, and R. Sommer (ALPHA Collaboration), J. High Energy Phys. 08 (2014) 150.

[48] S. Schaefer, R. Sommer, and F. Virotta (ALPHA Collaboration), Nucl. Phys. B 845, 93 (2011).

[49] K.-F. Liu, J. Liang, and Y.-B. Yang, Phys. Rev. D 97, 034507 (2018).

[50] W. H. Hockings and U. van Kolck, Phys. Lett. B 605, 273 (2005).

[51] A. Abdel-Rehim et al., Phys. Rev. D 92, 114513 (2015); 93, 039904(E) (2016).

[52] P. E. Shanahan, R. Horsley, Y. Nakamura, D. Pleiter, P. E. L. Rakow, G. Schierholz, H. Stuben, A. W. Thomas, R. D. Young, and J. M. Zanotti, Phys. Rev. D 89, 074511 (2014).

[53] F. J. M. Farley, K. Jungmann, J. P. Miller, W. M. Morse, Y. F. Orlov, B. L. Roberts, Y. K. Semertzidis, A. Silenko, and E. J. Stephenson, Phys. Rev. Lett. 93, 052001 (2004).

[54] G. W. Bennett et al. (Muon (g-2) Collaboration), Phys. Rev. D 80, 052008 (2009).

[55] G. Guidoboni et al. (JEDI Collaboration), Phys. Rev. Lett. 117, 054801 (2016).

[56] J. Bsaisou et al., Eur. Phys. J. A 49, 31 (2013).

[57] J. de Vries, P. Draper, K. Fuyuto, J. Kozaczuk, and D. Sutherland, Phys. Rev. D 99, 015042 (2019). 